\title{
EXTENSIONS OF TEMPERED REPRESENTATIONS
}

\author{
ERIC OPDAM AND MAARTEN SOLLEVELD
}

\begin{abstract}
Let $\pi, \pi^{\prime}$ be irreducible tempered representations of an affine Hecke algebra $\mathcal{H}$ with positive parameters. We compute the higher extension groups $\operatorname{Ext}_{\mathcal{H}}^{n}\left(\pi, \pi^{\prime}\right)$ explicitly in terms of the representations of analytic R-groups corresponding to $\pi$ and $\pi^{\prime}$. The result has immediate applications to the computation of the Euler-Poincaré pairing $E P\left(\pi, \pi^{\prime}\right)$, the alternating sum of the dimensions of the Ext-groups. The resulting formula for $E P\left(\pi, \pi^{\prime}\right)$ is equal to Arthur's formula for the elliptic pairing of tempered characters in the setting of reductive $p$-adic groups. Our proof applies equally well to affine Hecke algebras and to reductive groups over non-archimedean local fields of arbitrary characteristic. This sheds new light on the formula of Arthur and gives a new proof of Kazhdan's orthogonality conjecture for the Euler-Poincaré pairing of admissible characters.
\end{abstract}

\section{CONTENTS}

Introduction

1. Affine Hecke algebras

1.1. Parabolic induction

1.2. The Schwartz algebra

2. Formal completion of the Schwartz algebra 13

2.1. Exactness of formal completion 17

3. Algebras with finite group actions 19

3.1. Ext-groups and the Yoneda product 22

4. Analytic R-groups 26

5. Extensions of irreducible tempered modules 29

6. The Euler-Poincaré pairing 33

6.1. Arthur's formula

7. The case of reductive $p$-adic groups

References

\section{INTRODUCTION}

Let $\mathbb{F}$ be a non-archimedean local field, and let $L$ be the group of $\mathbb{F}$-rational points of a connected reductive algebraic group defined over $\mathbb{F}$. In this paper we will compute the vector space of higher extensions $\operatorname{Ext}_{L}^{n}\left(V, V^{\prime}\right)$ between smooth irreducible tempered representations $V, V^{\prime}$ of $L$ in the abelian category of smooth representations of $L$. In the formulation of the result a predominant role is played by the so called analytic $R$-groups which, by classical results due to Harish-Chandra, Knapp,

Date: October 28, 2018.

2010 Mathematics Subject Classification. Primary 20C08; Secondary 22E35, 22E50. 
Stein and Silberger, are fundamental in the classification of smooth irreducible tempered representations [HC, $\mathrm{KnSt}$, Sil]. The result applies to the computation of the Euler-Poincaré pairing for admissible tempered representations of $L$. In particular, this leads to a new proof for the "homological version" of Arthur's formula for the elliptic pairing of tempered characters of $L$ [Art, Ree] and to a new proof for Kazhdan's othogonality conjecture $\mathrm{Kaz}$ for the elliptic pairing of general admissible characters of $L$ (which was previously proved by Bezrukavnikov [Bez] and independently by Schneider and Stuhler [ScSt]).

The first and crucially important step is a result of Meyer Mey2 showing that there exists a natural isomorphism $\operatorname{Ext}_{\mathcal{H}(L)}^{n}\left(V, V^{\prime}\right) \simeq \operatorname{Ext}_{\mathcal{S}(L)}^{n}\left(V, V^{\prime}\right)$ where $\mathcal{S}(L)$ denotes the Harish-Chandra Schwartz algebra of $L$. Here $\mathcal{H}(L)$ and $\mathcal{S}(L)$ are considered as bornological algebras and the Ext-groups are defined in categories of bornological modules, see [Mey1. Recently the authors found an alternative proof of this result without the use of bornologies OpSo3.

The Schwartz algebra is a direct limit of the Fréchet subalgebras $\mathcal{S}(L, K)=$ $e_{K} \mathcal{S}(L) e_{K}$ of $K$-biinvariant functions in $\mathcal{S}(L)$, where $K$ runs over a system of "good" compact open subgroups of $L$. One can prove by Morita equivalence that $\operatorname{Ext}_{\mathcal{S}(L)}^{i}\left(V, V^{\prime}\right) \simeq \operatorname{Ext}_{\mathcal{S}(L, K)}^{i}\left(V^{K}, V^{\prime K}\right)$ if $V$ is generated by its $K$-invariant vectors (see Section 7). Now we use the structure of $\mathcal{S}(L, K)$ provided by Harish-Chandra's Fourier isomorphism Wal] in order to compute the right hand side. More precisely, we take the formal completion of the algebra $\mathcal{S}(L, K)$ at the central character of $V^{K}$, and show that this is Morita equivalent to a (twisted) crossed product of a formal power series ring with the analytic R-group. Finally, the Ext-groups of such algebras are easily computed using a Koszul resolution.

All ingredients necessary for the above line of arguments have been developed in detail in the context of abstract affine Hecke algebras as well DeOp1, DeOp2, OpSo1. The analytic R-groups are defined in terms of the Weyl group and the Plancherel density, all of which allow for explicit determination [Slo]. We will use the case of abstract affine Hecke algebras as the point of reference in this paper. In section 7 we will carefully formulate the results for the representation theory of $L$, and discuss the adaption of the arguments necessary for the proofs of those results.

The main technical difficulties to carry out the above steps arise from the necessity to control the properties of the formal completion functor in the context of the Fréchet algebras $\mathcal{S}(L, K)$, which are far from Noetherian. The category of bornological $\mathcal{S}(L, K)$-modules is a Quillen exact category Qui with respect to exact sequences that admit a bounded linear splitting. This implies (as used implicitly above) that the Ext-groups over the algebras $\mathcal{S}(L, K)$ are well-defined with respect to this exact structure. But the bounded linear splittings are not preserved when taking the formal completion at a central character, and this forces us to make careful choices of the exact categories with which to work.

We will now discuss the main results of this paper in more detail. Let us first recall some basic facts on analytic R-groups. Let $\mathcal{H}$ denote an abstract affine Hecke algebra with positive parameters. By [DeOp1 the category of tempered $\mathcal{H}$-modules of finite length decomposes into "blocks" which are parameterized by the set $\Xi_{\text {un }} / \mathcal{W}$ of orbits of tempered standard induction data $\xi \in \Xi_{\text {un }}$ for $\mathcal{H}$ under the action of the Weyl groupoid $\mathcal{W}$ for $\mathcal{H}$. To such a standard tempered induction datum $\xi \in \Xi_{\text {un }}$ one attaches a tempered standard induced module $\pi(\xi)$, which is a unitary tempered 
$\mathcal{H}$-module. The block $\operatorname{Mod}_{f, \mathcal{W} \xi}(\mathcal{S})$ of tempered modules associated with $\mathcal{W} \xi$ is generated by $\pi(\xi)$. The space $\Xi_{u n}$ of tempered induction data is a finite disjoint union of compact tori of various dimensions. In particular, at each $\xi \in \Xi_{\text {un }}$ there exists a well defined tangent space $T_{\xi}\left(\Xi_{u n}\right)$. With the action of the Weyl groupoid $\mathcal{W}$ we obtain a smooth orbifold $\Xi_{\text {un }} \rtimes \mathcal{W}$. The isotropy group $\mathcal{W}_{\xi} \subset \mathcal{W}$ of $\xi$ admits a canonical decomposition $\mathcal{W}_{\xi}=W\left(R_{\xi}\right) \rtimes \mathfrak{R}_{\xi}$. Here $W\left(R_{\xi}\right)$ is a real reflection group associated to an integral root system $R_{\xi}$ in $T_{\xi}\left(\Xi_{u n}\right)$ and $\mathfrak{R}_{\xi}$ is a group of diagram automorphisms with respect to a suitable choice of a basis of $R_{\xi}$. The subgroup $\mathfrak{R}_{\xi}$ is called the analytic R-group at $\xi$. Since $W\left(R_{\xi}\right)$ is a real reflection group, the quotient (in the category of complex affine varieties) $\left(T_{\xi}\left(\Xi_{\text {un }}\right) \otimes_{\mathbb{R}} \mathbb{C}\right) / W\left(R_{\xi}\right)$ is the complexification of a real vector space $E_{\xi}$ which carries a representation of $\mathfrak{R}_{\xi}$. The $\mathfrak{R}_{\xi}$-representation $E_{\xi}$ is independent of the choice of $\xi$ in its orbit $\mathcal{W} \xi$, up to equivalence.

The Knapp-Stein linear independence theorem (Theorem 4.1) for affine Hecke algebras DeOp2 states that the commutant of $\pi(\xi)(\mathcal{H})$ in $\operatorname{End}_{\mathbb{C}}(\pi(\xi))$ is isomorphic to the twisted group algebra $\mathbb{C}\left[\mathfrak{R}_{\xi}, \kappa_{\xi}\right]$ of the analytic R-group $\mathfrak{R}_{\xi}$, where $\kappa_{\xi}$ is a certain 2-cocycle. This sets up a bijection

$$
\begin{aligned}
\operatorname{Irr}\left(\mathbb{C}\left[\Re_{\xi}, \kappa_{\xi}\right]\right) & \longleftrightarrow \operatorname{Irr}_{\mathcal{W} \xi}(\mathcal{S}) \\
(\rho, V) & \longleftrightarrow \pi(\xi, \rho)
\end{aligned}
$$

between the set of irreducible representations of $\mathbb{C}\left[\mathfrak{R}_{\xi}, \kappa_{\xi}\right]$ and the set of irreducible objects in $\operatorname{Mod}_{f, \mathcal{W} \xi}(\mathcal{S})$. Hence the collection $\pi(\xi, \rho)$ where $\mathcal{W} \xi$ runs over the set of $\mathcal{W}$-orbits in $\Xi_{\text {un }}$ and $\rho$ runs over the set of irreducible representations of $\mathbb{C}\left[\Re_{\xi}, \kappa_{\xi}\right]$ is a complete set of representatives for the equivalence classes of tempered irreducible representations of $\mathcal{H}$.

Let $\mathfrak{R}_{\xi}^{*}$ be the Schur extension of $\mathfrak{R}_{\xi}$ and let $p_{\xi} \in \mathbb{C}\left[\mathfrak{R}_{\xi}^{*}\right]$ be the central idempotent corresponding to the two-sided ideal $\mathbb{C}\left[\mathfrak{R}_{\xi}, \kappa_{\xi}^{-1}\right] \subset \mathbb{C}\left[\mathfrak{R}_{\xi}^{*}\right]$. The first main result of this paper is a generalization of the Knapp-Stein Theorem. It establishes an equivalence between the category of $\mathcal{S}$-modules with generalized central character $\mathcal{W} \xi$ and the module category of the complex algebra

$$
\mathcal{A}_{\xi}:=p_{\xi}\left(\widehat{S\left(E_{\xi}\right)} \rtimes \mathfrak{R}_{\xi}^{*}\right) \cong\left(\widehat{S\left(E_{\xi}\right)} \otimes \operatorname{End}_{\mathbb{C}}\left(p_{\xi} \mathbb{C}\left[\mathfrak{R}_{\xi}^{*}\right]\right)\right)^{\mathfrak{R}_{\xi}^{*}} .
$$

Here $S\left(E_{\xi}\right)$ denotes the algebra of complex valued polynomial functions on $E_{\xi}^{*}$ and $\widehat{S\left(E_{\xi}\right)}$ is its formal completion at $0 \in E_{\xi}^{*}$. The group $\mathfrak{R}_{\xi}^{*}$ acts on this space through its natural quotient $\mathfrak{R}_{\xi}$. Consider the $\mathcal{A}_{\xi}$-module

$$
D:=\left(\widehat{S\left(E_{\xi}\right)} \otimes \operatorname{Hom}_{\mathbb{C}}\left(\pi(\xi), p_{\xi} \mathbb{C}\left[\Re_{\xi}^{*}\right]\right)\right)^{\mathfrak{R}_{\xi}^{*}} .
$$

The Fourier isomorphism (23) from [DeOp1, Theorem 5.3] implies that $D$ also admits a natural right $\mathcal{S}$-module structure, turning it into a $\mathcal{A}_{\xi} \mathcal{S}$-bimodule. To make the above precise we consider the categories $\operatorname{Mod}_{\text {bor }}\left(\mathcal{A}_{\xi}\right)$ of bornological $\mathcal{A}_{\xi}$-modules and $\operatorname{Mod}_{\text {bor }}^{\mathcal{W} \xi \text { tor }}(\mathcal{S})$, which consists of the bornological $\mathcal{S}$-modules that are annihilated by all $\mathcal{W}$-invariant smooth functions on $\Xi_{\text {un }}$ that are flat in $\xi$. In both cases the bornology is derived from the Fréchet algebra structure. We endow these categories with the exact structure of module extensions with a bounded linear splitting.

Instead of this bornological machinery, one may also think of Fréchet modules and exact sequences that admit continuous linear splittings. Indeed, the functor 
$\operatorname{Mod}_{F r}(\mathcal{S}) \rightarrow \operatorname{Mod}_{b o r}(\mathcal{S})$ that equips a Fréchet $\mathcal{S}$-module with its precompact bornology is a fully exact embedding OpSo1, Proposition A.2]. Hence all our constructions could be viewed purely within the context of Frechet $\mathcal{S}$-modules and that would yield the same results, restricted to this fully exact subcategory of the category of bornological $\mathcal{S}$-modules.

Theorem 1. (See Theorem 5.1.)

There is an equivalence of exact categories

$$
\operatorname{Mod}_{b o r}^{\mathcal{V} \xi, t o r}(\mathcal{S}) \rightarrow \operatorname{Mod}_{\text {bor }}\left(\mathcal{A}_{\xi}\right), M \mapsto D \widehat{\otimes}_{\mathcal{S}} M,
$$

and similarly for the corresponding categories of Fréchet modules.

The restriction of this result to irreducible modules essentially recovers the aforementioned Knapp-Stein Theorem. The proof of Theorem 1 is analytic in nature, some of the ideas are already present in [Was, LePl].

Since the algebra $\mathcal{A}_{\xi}$ is a twisted crossed product of a formal power series ring with $\mathfrak{R}_{\xi}$, it is not hard to compute the Ext-groups between modules in $\operatorname{Mod}_{f, \mathcal{W} \xi}(\mathcal{S})$ using Theorem 1, But we are more interested in computing their Ext-groups in the category of $\mathcal{H}$-modules or equivalently Mey1, OpSo1 in $\operatorname{Mod}_{b o r}(\mathcal{S})$. To go from there to $\operatorname{Mod}_{\text {bor }}^{\mathcal{W} \xi \text {,tor }}(\mathcal{S})$ boils down to applying the formal completion functor at a central character. In Section 2 we show that this completion functor is exact and preserves Ext-groups in suitable module categories. The underlying reason is that the Taylor series map from smooth functions to power series induces an exact functor on finitely generated modules [ToMe. With that, Theorem 1 and an explicit Koszul resolution for $\mathcal{A}_{\xi}$ we can calculate the Ext-groups for irreducible tempered $\mathcal{H}$-representations:

Theorem 2. (See Theorem 5.2.)

Let $(\rho, V),\left(\rho^{\prime}, V^{\prime}\right) \in \operatorname{Irr}\left(\mathbb{C}\left[\Re_{\xi}, \kappa_{\xi}\right]\right)$ and let $n \geq 0$. Then

$$
\operatorname{Ext}_{\mathcal{H}}^{n}\left(\pi(\xi, \rho), \pi\left(\xi, \rho^{\prime}\right)\right) \cong\left(\operatorname{Hom}_{\mathbb{C}}\left(V^{\prime}, V\right) \otimes_{\mathbb{R}} \bigwedge^{n} E_{\xi}^{*}\right)^{\mathfrak{R}_{\xi}}
$$

Since $\mathcal{S}$-modules with different central characters have only trivial extensions, Theorem 2 is the essential case for the determination of extensions between finite dimensional $\mathcal{S}$-modules. Our proof also applies in the context of tempered representations of reductive $p$-adic groups:

Theorem 3. (See Section [7.)

Let $\mathbf{L}$ be a connected reductive group defined over a non-archimedean local field $\mathbb{F}$. Then the analogues of Theorems 1 and 0 holds for tempered irreducible representations of $L=\mathbf{L}(\mathbb{F})$.

These results have obvious consequences for the computation of the Euler-Poincaré pairings. For any $\mathbb{C}$-linear abelian category $\mathcal{C}$ with finite homological dimension one may define the Euler-Poincaré pairing [ScSt] of two objects of finite length $\pi, \pi^{\prime} \in \operatorname{Obj}(\mathcal{C})$ by the formula

$$
E P_{\mathcal{C}}\left(\pi, \pi^{\prime}\right)=\sum_{n \geq 0}(-1)^{n} \operatorname{dim}_{\mathbb{C}} \operatorname{Ext}_{\mathcal{C}}^{n}\left(\pi, \pi^{\prime}\right)
$$

provided that the dimensions of all the Ext-groups between irreducible objects are finite. Let $K_{\mathbb{C}}(\mathcal{C})$ denote the Grothendieck group (tensored by $\mathbb{C}$ ) of finite length 
objects in $\mathcal{C}$. The Euler-Poincaré pairing extends to a sesquilinear form $E P$ on $K_{\mathbb{C}}(\mathcal{C})$.

A well known instance of this construction is the Euler-Poincaré pairing on the Grothendieck group $G_{\mathbb{C}}(L)$ of admissible representations of $L$ [BeDe, [ScSt]. In this case $\mathcal{C}$ is the category of smooth representations of $L$. The resulting pairing $E P_{L}$ is Hermitian ([ScSt]; See Proposition 7.2 for a more direct argument) and plays a fundamental role in the local trace formula and in the study of orbital integrals on the regular elliptic set of $L$ Art, ScSt, Bez, Ree. The definition of EP also applies naturally to the Grothendieck group $G_{\mathbb{C}}(\mathcal{H})$ of finite dimensional representations of an abstract affine Hecke algebra $\mathcal{H}$ with positive parameters. Here one uses that the category of finitely generated $\mathcal{H}$-modules has finite cohomological dimension by OpSo1. The form $E P_{\mathcal{H}}$ on $G_{\mathbb{C}}(\mathcal{H})$ is also Hermitian OpSo1, Theorem 3.5.a].

Theorem 2 implies the following formula for the Euler-Poincaré pairing between irreducible tempered representations of an affine Hecke algebra $\mathcal{H}$ (see Theorem 6.5):

$$
E P_{\mathcal{H}}\left(\pi(\xi, \rho), \pi\left(\xi, \rho^{\prime}\right)\right)=\left|\Re_{\xi}\right|^{-1} \sum_{r \in \Re_{\xi}}|d(r)| \operatorname{tr}_{\rho}(r) \overline{\operatorname{tr}_{\rho^{\prime}}(r)} .
$$

where $d(r)=\operatorname{det}_{T_{\xi}\left(\Xi_{u n}\right)}(1-r)$. Similarly Theorem 3 implies the analog of (2) in the context of tempered representations of a reductive $p$-adic group $L$. We formulate this as a theorem since it had not yet been established in this generality:

Theorem 4. (See (91))

Let $\mathbf{L}$ be a connected reductive group defined over a non-archimedean local field $\mathbb{F}$. Then the analog of (2) holds for the Euler-Poincaré pairing of tempered irreducible representations of $L=\mathbf{L}(\mathbb{F})$.

In the case of Iwahori-spherical representations of split groups this result was previously shown by Reeder Ree] using different arguments. If $\operatorname{char}(\mathbb{F})=0$ then Theorem 4 can alternatively be derived by combining Arthur's formula Art, Corollary 6.3] for the elliptic pairing of tempered characters with Kazhdan's orthogonality conjecture for the Euler-Poincaré pairing of admissible characters of $L$, which was proved independently in [ScSt, Theorem III.4.21] and in [Bez, Theorem 0.20]. Interestingly, this argument can also be reversed showing that Theorem 4 gives rise to a new proof of Kazhdan's orthogonality conjecture. We explain this argument more precisely now.

Let us recall the elliptic pairing of admissible characters. Suppose that the ground field $\mathbb{F}$ of $L$ has characteristic 0 and let $C^{\text {ell }}$ be the set of regular semisimple elliptic conjugacy classes of $L$. By definition $C^{e l l}$ is empty if the center $Z(L)$ of $L$ is not compact. There exists a canonical elliptic measure $d \gamma$ (the "Weyl measure") on $C^{\text {ell }} \mathrm{Kaz}$. Let $\theta_{\pi}$ denote the locally constant function on $C^{\text {ell }}$ determined by the distributional character of an admissible representation $\pi$. The elliptic pairing of $\pi$ and $\pi^{\prime}$ is defined as

$$
e_{L}\left(\pi, \pi^{\prime}\right):=\int_{C^{e l l}} \theta_{\pi}\left(c^{-1}\right) \theta_{\pi^{\prime}}(c) \mathrm{d} \gamma(c) .
$$

Kazhdan's orthogonality conjecture for the Euler-Poincaré pairing of admissible characters of $L$ states that, under the assumptions made above, the elliptic pairing $e_{L}\left(\pi, \pi^{\prime}\right)$ of two admissible representations $\pi$ and $\pi^{\prime}$ of $L$ is equal to their EulerPoincaré pairing:

$$
e_{L}\left(\pi, \pi^{\prime}\right)=E P_{L}\left(\pi, \pi^{\prime}\right)
$$


Next we indicate how (44) also follows from Theorem 4. The connection is provided by results of Arthur on the elliptic pairing of tempered characters. Let $P \subset L$ be a parabolic subgroup with Levi component $M$, and let $\sigma$ be a smooth irreducible representation of $M$, square integrable modulo the center of $M$. The representation $\mathcal{I}_{P}^{L}(\sigma)$, the smooth normalized parabolically induced representation from $\sigma$, is a tempered admissible unitary representation of $L$. The decomposition of $\mathcal{I}_{P}^{L}(\sigma)$ is governed by the associated analytic R-group $\mathfrak{R}_{\sigma}$, a finite group which acts naturally on the real Lie algebra $\operatorname{Hom}\left(X^{*}(M), \mathbb{R}\right)$ of the center of $M$. For $r \in \mathfrak{R}_{\sigma}$ we denote by $d(r)$ the determinant of the linear transformation $1-r$ on $\operatorname{Hom}\left(X^{*}(M), \mathbb{R}\right)$. We note that $d(r)=0$ whenever $Z(L)$ is not compact.

Let $\pi$ be an irreducible tempered representation of $L$ which occurs in $\mathcal{I}_{P}^{L}(\sigma)$, which we denote by $\pi \prec \mathcal{I}_{P}^{L}(\sigma)$. The theory of the analytic R-group asserts that $\rho=\operatorname{Hom}_{L}\left(\pi, \mathcal{I}_{P}^{L}(\sigma)\right)$ is an irreducible projective $\mathfrak{R}_{\sigma}$-representation. It was shown in [Art, Corollary 6.3] that for all tempered irreducible representations $\pi, \pi^{\prime} \prec \mathcal{I}_{P}^{L}(\sigma)$ one has

$$
e_{L}\left(\pi, \pi^{\prime}\right)=\left|\Re_{\sigma}\right|^{-1} \sum_{r \in \Re_{\sigma}}|d(r)| \operatorname{tr}_{\rho}(r) \overline{\operatorname{tr}_{\rho^{\prime}}(r)} .
$$

The proof uses the local trace formula for $L$, which requires that $\operatorname{char}(\mathbb{F})=0$. With our conventions (5) is trivial when $Z(L)$ is not compact, Arthur has a more sophisticated equality in that situation. If $\pi$ and $\pi^{\prime}$ do not arise (up to equivalence) as components of the same standard induced representation $\mathcal{I}_{P}^{L}(\sigma)$, then their elliptic pairing is zero. From (5) and Theorem 4 it is only a short trip to a proof of Kazhdan's orthogonality conjecture:

Theorem 5. (See Theorem 7.3.)

Let $\mathbf{L}$ be a connected reductive group defined over a non-archimedean local field $\mathbb{F}$ of characteristic 0 . Then equation (4) holds for all admissible representations $\pi, \pi^{\prime}$ of $L=\mathbf{L}(\mathbb{F})$.

The elliptic pairing and the set $C^{\text {ell }}$ of elliptic conjugacy classes in (3) do not seem to have obvious counterparts in the setting of affine Hecke algebras. Reeder Ree introduced the elliptic pairing for the cross product of a finite group with a real representation. This construction was extended in OpSo1, Theorem 3.3] to the case of a finite group acting on a lattice. To relate these notions of elliptic pairing for Weyl groups to the Euler-Poincaré characteristic $E P_{\mathcal{H}}$ one compares $E P_{\mathcal{H}}$ and $E P_{W}$, which is done in [Ree, Section 5.6] (for affine Hecke algebras with equal parameters) and in OpSo1, Chapter 3]. Recent results from [Sol3 allow us to conclude that $G_{\mathbb{C}}(\mathcal{H})$ modulo the radical of $E P_{\mathcal{H}}$ equals the vector space $\operatorname{Ell}(\mathcal{H})$ of "elliptic characters", and that this space does not depend on the Hecke parameters $q$ (Theorem 6.4).

Arthur's explicit formula (2) for $E P_{\mathcal{H}}$ applies only to the Euler-Poincaré pairing of tempered characters. In an abstract sense this no restriction, as it follows from the Langlands classification of irreducible characters of $\mathcal{H}$ in terms of standard induction data in Langlands position, that modulo the radical of the pairing $E P_{\mathcal{H}}$ any irreducible character is equivalent to a virtual tempered character. But this is complicated in practice, and therefore our formula does not qualify as an explicit formula for $E P_{\mathcal{H}}$ for general non-tempered irreducible characters. It would therefore be desirable to extend the result to general finite dimensional representations of $\mathcal{H}$. 
In Section 4 we make a first step by extending the definition of the analytic $R$-group to non-tempered induction data. We show that its irreducible characters are in natural bijection with the Langlands quotients associated to the induction datum. However, we have not been able to generalize the Arthur formula to this case. It seems that one would need an appropriate version of Kazhdan-Lusztig polynomials to address the problem in this generality.

\section{Affine Hecke algebras}

Here we recall the definitions and notations of our most important objects of study. Several things described in this section can be found in more detail elsewhere in the literature, see in particular [Lus1, Opd1, OpSo1, Sol3].

Let $\mathfrak{a}$ be a finite dimensional real vector space and let $\mathfrak{a}^{*}$ be its dual. Let $Y \subset \mathfrak{a}$ be a lattice and $X=\operatorname{Hom}_{\mathbb{Z}}(Y, \mathbb{Z}) \subset \mathfrak{a}^{*}$ the dual lattice. Let

$$
\mathcal{R}=\left(X, R_{0}, Y, R_{0}^{\vee}, F_{0}\right) .
$$

be a based root datum. Thus $R_{0}$ is a reduced root system in $X, R_{0}^{\vee} \subset Y$ is the dual root system, $F_{0}$ is a basis of $R_{0}$ and the set of positive roots is denoted $R_{0}^{+}$. Furthermore we are given a bijection $R_{0} \rightarrow R_{0}^{\vee}, \alpha \mapsto \alpha^{\vee}$ such that $\left\langle\alpha, \alpha^{\vee}\right\rangle=2$ and such that the corresponding reflections $s_{\alpha}: X \rightarrow X$ (resp. $s_{\alpha}^{\vee}: Y \rightarrow Y$ ) stabilize $R_{0}$ (resp. $R_{0}^{\vee}$ ). We do not assume that $R_{0}$ spans $\mathfrak{a}^{*}$.

The reflections $s_{\alpha}$ generate the Weyl group $W_{0}=W\left(R_{0}\right)$ of $R_{0}$, and $S_{0}:=\left\{s_{\alpha}\right.$ : $\left.\alpha \in F_{0}\right\}$ is the collection of simple reflections. We have the affine Weyl group $W^{\text {aff }}=\mathbb{Z} R_{0} \rtimes W_{0}$ and the extended (affine) Weyl group $W=X \rtimes W_{0}$. Both can be regarded as groups of affine transformations of $\mathfrak{a}^{*}$. We denote the translation corresponding to $x \in X$ by $t_{x}$.

As is well known, $W^{\text {aff }}$ is a Coxeter group, and the basis of $R_{0}$ gives rise to a set $S^{\text {aff }}$ of simple (affine) reflections. The length function $\ell$ of the Coxeter system ( $W^{\text {aff }}, S^{\text {aff }}$ ) extends naturally to $W$, by counting how many negative roots of the affine root system $R_{0}^{\vee} \times \mathbb{Z}$ are made positive by an element of $W$. We write

$$
\begin{aligned}
& X^{+}:=\left\{x \in X:\left\langle x, \alpha^{\vee}\right\rangle \geq 0 \forall \alpha \in F_{0}\right\}, \\
& X^{-}:=\left\{x \in X:\left\langle x, \alpha^{\vee}\right\rangle \leq 0 \forall \alpha \in F_{0}\right\}=-X^{+} .
\end{aligned}
$$

It is easily seen that the center of $W$ is the lattice

$$
Z(W)=X^{+} \cap X^{-} .
$$

We say that $\mathcal{R}$ is semisimple if $Z(W)=0$ or equivalently if $R_{0}$ spans $\mathfrak{a}^{*}$. Thus a root datum is semisimple if and only if the corresponding reductive algebraic group is so.

With $\mathcal{R}$ we also associate some other root systems. There is the non-reduced root system

$$
R_{n r}:=R_{0} \cup\left\{2 \alpha: \alpha^{\vee} \in 2 Y\right\} .
$$

Obviously we put $(2 \alpha)^{\vee}=\alpha^{\vee} / 2$. Let $R_{1}$ be the reduced root system of long roots in $R_{n r}$ :

$$
R_{1}:=\left\{\alpha \in R_{n r}: \alpha^{\vee} \notin 2 Y\right\}
$$


Consider a positive parameter function for $\mathcal{R}$, that is, a function $q: W \rightarrow \mathbb{R}_{>0}$ such that

$$
\begin{array}{lll}
q(\omega)=1 & \text { if } \ell(\omega)=0, \\
q(w v)=q(w) q(v) & \text { if } w, v \in W \quad \text { and } \quad \ell(w v)=\ell(w)+\ell(v) .
\end{array}
$$

Alternatively it can be given by $W_{0}$-invariant map $q: R_{n r}^{\vee} \rightarrow \mathbb{R}_{>0}$, the relation being

$$
\begin{array}{lll}
q_{\alpha \vee}=q\left(s_{\alpha}\right)=q\left(t_{\alpha} s_{\alpha}\right) & \text { if } & \alpha \in R_{0} \cap R_{1}, \\
q_{\alpha \vee}=q\left(t_{\alpha} s_{\alpha}\right) & \text { if } & \alpha \in R_{0} \backslash R_{1}, \\
q_{\alpha \vee / 2}=q\left(s_{\alpha}\right) q\left(t_{\alpha} s_{\alpha}\right)^{-1} & \text { if } & \alpha \in R_{0} \backslash R_{1} .
\end{array}
$$

In case $R_{0}$ is irreducible this means that $q$ is determined by one, two or three independent real numbers, where three only occurs for a root datum of type $C_{n}^{(1)}$. The affine Hecke algebra $\mathcal{H}=\mathcal{H}(\mathcal{R}, q)$ is the unique associative complex algebra with basis $\left\{N_{w}: w \in W\right\}$ and multiplication rules

$$
\begin{array}{ll}
N_{w} N_{v}=N_{w v} & \text { if } \quad \ell(w v)=\ell(w)+\ell(v), \\
\left(N_{s}-q(s)^{1 / 2}\right)\left(N_{s}+q(s)^{-1 / 2}\right)=0 & \text { if } \quad s \in S^{\text {aff }} .
\end{array}
$$

In the literature one also finds this algebra defined in terms of the elements $q(s)^{1 / 2} N_{s}$, in which case the multiplication can be described without square roots. The algebra $\mathcal{H}$ is endowed with a conjugate-linear involution, defined on basis elements by $N_{w}^{*}:=$ $N_{w^{-1}}$.

For $x \in X^{+}$we put $\theta_{x}:=N_{t_{x}}$. The corresponding semigroup morphism $X^{+} \rightarrow$ $\mathcal{H}(\mathcal{R}, q)^{\times}$extends to a group homomorphism

$$
X \rightarrow \mathcal{H}(\mathcal{R}, q)^{\times}: x \mapsto \theta_{x} .
$$

A part of the Bernstein presentation [Lus1, §3] says that the subalgebra $\mathcal{A}:=$ $\operatorname{span}\left\{\theta_{x}: x \in X\right\}$ is isomorphic to $\mathbb{C}[X]$, and that the center $Z(\mathcal{H})$ corresponds to $\mathbb{C}[X]^{W_{0}}$ under this isomorphism. Let $T$ be the complex algebraic torus

$$
T=\operatorname{Hom}_{\mathbb{Z}}\left(X, \mathbb{C}^{\times}\right) \cong Y \otimes_{\mathbb{Z}} \mathbb{C}^{\times},
$$

so $\mathcal{A} \cong \mathcal{O}(T)$ and $Z(\mathcal{H})=\mathcal{A}^{W_{0}} \cong \mathcal{O}\left(T / W_{0}\right)$. This torus admits a polar decomposition

$$
T=T_{r s} \times T_{u n}=\operatorname{Hom}_{\mathbb{Z}}\left(X, \mathbb{R}_{>0}\right) \times \operatorname{Hom}_{\mathbb{Z}}\left(X, S^{1}\right)
$$

into a real split (or positive) part and a unitary part.

Let $\operatorname{Mod}_{f}(\mathcal{H})$ be the category of finite dimensional $\mathcal{H}$-modules, and $\operatorname{Mod}_{f, W_{0} t}(\mathcal{H})$ the full subcategory of modules that admit the $Z(\mathcal{H})$-character $W_{0} t \in T / W_{0}$. We let $G(\mathcal{H})$ be the Grothendieck group of $\operatorname{Mod}_{f}(\mathcal{H})$ and we write $G_{\mathbb{C}}(\mathcal{H})=\mathbb{C} \otimes_{\mathbb{Z}} G(\mathcal{H})$. Furthermore we denote by $\operatorname{Irr}(\mathcal{H})$, respectively $\operatorname{Irr}_{W_{0} t}(\mathcal{H})$, the set of equivalence classes of irreducible objects in $\operatorname{Mod}_{f}(\mathcal{H})$, respectively $\operatorname{Mod}_{f, W_{0} t}(\mathcal{H})$. We will use these notations also for other algebras and groups.

1.1. Parabolic induction. For a set of simple roots $P \subset F_{0}$ we introduce the notations

$$
\begin{array}{ll}
R_{P}=\mathbb{Q} P \cap R_{0} & R_{P}^{\vee}=\mathbb{Q} P^{\vee} \cap R_{0}^{\vee}, \\
X_{P}=X /\left(X \cap\left(P^{\vee}\right)^{\perp}\right) & X^{P}=X /(X \cap \mathbb{Q} P), \\
Y_{P}=Y \cap \mathbb{Q} P^{\vee} & Y^{P}=Y \cap P^{\perp}, \\
\mathfrak{a}_{P}=\mathbb{R} P^{\vee} & \mathfrak{a}^{P}=P^{\perp}, \\
T_{P}=\operatorname{Hom}_{\mathbb{Z}}\left(X_{P}, \mathbb{C}^{\times}\right) & T^{P}=\operatorname{Hom}_{\mathbb{Z}}\left(X^{P}, \mathbb{C}^{\times}\right), \\
\mathcal{R}_{P}=\left(X_{P}, R_{P}, Y_{P}, R_{P}^{\vee}, P\right) & \mathcal{R}^{P}=\left(X, R_{P}, Y, R_{P}^{\vee}, P\right) .
\end{array}
$$


Notice that $T_{P}$ and $T^{P}$ are algebraic subtori of $T$. Although $T_{r s}=T_{P, r s} \times T_{r s}^{P}$, the product $T_{u n}=T_{P, u n} T_{u n}^{P}$ is not direct, because the intersection

$$
T_{P, u n} \cap T_{u n}^{P}=T_{P} \cap T^{P}
$$

can have more than one element (but only finitely many).

We define parameter functions $q_{P}$ and $q^{P}$ on the root data $\mathcal{R}_{P}$ and $\mathcal{R}^{P}$, as follows. Restrict $q$ to a function on $\left(R_{P}\right)_{n r}^{\vee}$ and use (7) to extend it to $W\left(\mathcal{R}_{P}\right)$ and $W\left(\mathcal{R}^{P}\right)$. Now we can define the parabolic subalgebras

$$
\mathcal{H}_{P}=\mathcal{H}\left(\mathcal{R}_{P}, q_{P}\right), \quad \mathcal{H}^{P}=\mathcal{H}\left(\mathcal{R}^{P}, q^{P}\right) .
$$

Despite our terminology $\mathcal{H}^{P}$ and $\mathcal{H}_{P}$ are not subalgebras of $\mathcal{H}$, but they are close. Namely, $\mathcal{H}\left(\mathcal{R}^{P}, q^{P}\right)$ is isomorphic to the subalgebra of $\mathcal{H}(\mathcal{R}, q)$ generated by $\mathcal{A}$ and $\mathcal{H}\left(W\left(R_{P}\right), q_{P}\right)$. We denote the image of $x \in X$ in $X_{P}$ by $x_{P}$ and we let $\mathcal{A}_{P} \subset \mathcal{H}_{P}$ be the commutative subalgebra spanned by $\left\{\theta_{x_{P}}: x_{P} \in X_{P}\right\}$. There is natural surjective quotient map

$$
\mathcal{H}^{P} \rightarrow \mathcal{H}_{P}: \theta_{x} N_{w} \mapsto \theta_{x_{P}} N_{w}
$$

For all $x \in X$ and $\alpha \in P$ we have

$$
x-s_{\alpha}(x)=\left\langle x, \alpha^{\vee}\right\rangle \alpha \in \mathbb{Z} P,
$$

so $t\left(s_{\alpha}(x)\right)=t(x)$ for all $t \in T^{P}$. Hence $t(w(x))=t(x)$ for all $w \in W\left(R_{P}\right)$, and we can define an algebra automorphism

$$
\phi_{t}: \mathcal{H}^{P} \rightarrow \mathcal{H}^{P}, \quad \phi_{t}\left(\theta_{x} N_{w}\right)=t(x) \theta_{x} N_{w} \quad t \in T^{P} .
$$

In particular, for $t \in T_{P} \cap T^{P}$ this descends to an algebra automorphism

$$
\psi_{t}: \mathcal{H}_{P} \rightarrow \mathcal{H}_{P}, \quad \theta_{x_{P}} N_{w} \mapsto t\left(x_{P}\right) \theta_{x_{P}} N_{w} \quad t \in T_{P} \cap T^{P} .
$$

Suppose that $g \in W_{0}$ satisfies $g(P)=Q \subseteq F_{0}$. Then there are algebra isomorphisms

$$
\begin{aligned}
& \psi_{g}: \mathcal{H}_{P} \rightarrow \mathcal{H}_{Q}, \quad \theta_{x_{P}} N_{w} \\
& \psi_{g}: \mathcal{H}^{P} \rightarrow \mathcal{H}^{Q}, \quad \theta_{x} N_{w} \mapsto \theta_{g x} N_{g w g^{-1}} N_{g w g^{-1}},
\end{aligned}
$$

We can regard any representation $\left(\sigma, V_{\sigma}\right)$ of $\mathcal{H}\left(\mathcal{R}_{P}, q_{P}\right)$ as a representation of $\mathcal{H}\left(\mathcal{R}^{P}, q^{P}\right)$ via the quotient map (10). Thus we can construct the $\mathcal{H}$-representation

$$
\pi(P, \sigma, t):=\operatorname{Ind}_{\mathcal{H}\left(\mathcal{R}^{P}, q^{P}\right)}^{\mathcal{H}(\mathcal{R}, q)}\left(\sigma \circ \phi_{t}\right) .
$$

Representations of this form are said to be parabolically induced.

We intend to partition $\operatorname{Irr}(\mathcal{H})$ into finite packets, each of which is obtained by inducing a discrete series representation of a parabolic subalgebra of $\mathcal{H}$. The discrete series and tempered representations are defined via the $\mathcal{A}$-weights of a representation, as we recall now. Given $P \subseteq F_{0}$, we have the following positive cones in $\mathfrak{a}$ and in $T_{r s}$ :

$$
\begin{aligned}
& \mathfrak{a}^{+} \quad=\left\{\mu \in \mathfrak{a}:\langle\alpha, \mu\rangle \geq 0 \forall \alpha \in F_{0}\right\}, \quad T^{+}=\exp \left(\mathfrak{a}^{+}\right), \\
& \mathfrak{a}_{P}^{+} \quad=\left\{\mu \in \mathfrak{a}_{P}:\langle\alpha, \mu\rangle \geq 0 \forall \alpha \in P\right\}, \quad T_{P}^{+} \quad=\exp \left(\mathfrak{a}_{P}^{+}\right), \\
& \mathfrak{a}^{P+}=\left\{\mu \in \mathfrak{a}^{P}:\langle\alpha, \mu\rangle \geq 0 \forall \alpha \in F_{0} \backslash P\right\}, \quad T^{P+} \quad=\exp \left(\mathfrak{a}^{P+}\right), \\
& \mathfrak{a}^{P++}=\left\{\mu \in \mathfrak{a}^{P}:\langle\alpha, \mu\rangle>0 \forall \alpha \in F_{0} \backslash P\right\}, \quad T^{P++}=\exp \left(\mathfrak{a}^{P++}\right) \text {. }
\end{aligned}
$$

The antidual of $\mathfrak{a}^{*+}:=\left\{x \in \mathfrak{a}^{*}:\left\langle x, \alpha^{\vee}\right\rangle \geq 0 \forall \alpha \in F_{0}\right\}$ is

$$
\mathfrak{a}^{-}=\left\{\lambda \in \mathfrak{a}:\langle x, \lambda\rangle \leq 0 \forall x \in \mathfrak{a}^{*+}\right\}=\left\{\sum_{\alpha \in F_{0}} \lambda_{\alpha} \alpha^{\vee}: \lambda_{\alpha} \leq 0\right\} .
$$


Similarly we define

$$
\mathfrak{a}_{P}^{-}=\left\{\sum_{\alpha \in P} \lambda_{\alpha} \alpha^{\vee} \in \mathfrak{a}_{P}: \lambda_{\alpha} \leq 0\right\} .
$$

The interior $\mathfrak{a}^{--}$of $\mathfrak{a}^{-}$equals $\left\{\sum_{\alpha \in F_{0}} \lambda_{\alpha} \alpha^{\vee}: \lambda_{\alpha}<0\right\}$ if $F_{0}$ spans $\mathfrak{a}^{*}$, and is empty otherwise. We write $T^{-}=\exp \left(\mathfrak{a}^{-}\right)$and $T^{--}=\exp \left(\mathfrak{a}^{--}\right)$.

Let $t=|t| \cdot t|t|^{-1} \in T_{r s} \times T_{u n}$ be the polar decomposition of $t$. An $\mathcal{H}$-representation is called tempered if $|t| \in T^{-}$for all its $\mathcal{A}$-weights $t$, and anti-tempered if $|t|^{-1} \in T^{-}$ for all such $t$. More restrictively we say that an irreducible $\mathcal{H}$-representation belongs to the discrete series (or simply: is discrete series) if $|t| \in T^{--}$, for all its $\mathcal{A}$-weights $t$. In particular the discrete series is empty if $F_{0}$ does not span $\mathfrak{a}^{*}$.

Our induction data are triples $(P, \delta, t)$, where

- $P \subset F_{0}$;

- $\left(\delta, V_{\delta}\right)$ is a discrete series representation of $\mathcal{H}_{P}$;

- $t \in T^{P}$.

Let $\Xi$ be the space of such induction data, where we consider $\delta$ only modulo equivalence of $\mathcal{H}_{P}$-representations. We say that $\xi=(P, \delta, t)$ is unitary if $t \in T_{u n}^{P}$, and we denote the space of unitary induction data by $\Xi_{u n}$. Similarly we say that $\xi$ is positive if $|t| \in T^{P+}$, which we write as $\xi \in \Xi^{+}$. We have three collections of induction data:

$$
\Xi_{\text {un }} \subseteq \Xi^{+} \subseteq \Xi \text {. }
$$

By default we endow these spaces with the topology for which $P$ and $\delta$ are discrete variables and $T^{P}$ carries its natural analytic topology. With $\xi \in \Xi$ we associate the parabolically induced representation

$$
\pi(\xi)=\pi(P, \delta, t):=\operatorname{Ind}_{\mathcal{H}^{P}}^{\mathcal{H}}\left(\delta \circ \phi_{t}\right) .
$$

As vector space underlying $\pi(\xi)$ we will always take $\mathbb{C}\left[W^{P}\right] \otimes V_{\delta}$, where $W^{P}$ is the collection of shortest length representatives of $W_{0} / W\left(R_{P}\right)$. This space does not depend on $t$, which will allow us to speak of maps that are continuous, smooth, polynomial or even rational in the parameter $t \in T^{P}$. It is known that $\pi(\xi)$ is unitary and tempered if $\xi \in \Xi_{u n}$, and non-tempered if $\xi \in \Xi \backslash \Xi_{u n}$, see Opd1, Propositions 4.19 and 4.20] and [Sol3, Lemma 3.1.1].

The relations between such representations are governed by intertwining operators. Their construction Opd1 is rather complicated, so we recall only their important properties. Suppose that $P, Q \subset F_{0}, k \in T_{P} \cap T^{P}, w \in W_{0}$ and $w(P)=Q$. Let $\delta$ and $\sigma$ be discrete series representations of respectively $\mathcal{H}_{P}$ and $\mathcal{H}_{Q}$, such that $\sigma$ is equivalent with $\delta \circ \psi_{k}^{-1} \circ \psi_{w}^{-1}$.

Theorem 1.1. Opd1, Theorem 4.33 and Corollary 4.34]

(a) There exists a family of intertwining operators

$$
\pi(w k, P, \delta, t): \pi(P, \delta, t) \rightarrow \pi(Q, \sigma, w(k t)) .
$$

As a map $\mathbb{C}\left[W^{P}\right] \otimes_{\mathbb{C}} V_{\delta} \rightarrow \mathbb{C}\left[W^{Q}\right] \otimes_{\mathbb{C}} V_{\sigma}$ it is a rational in $t \in T^{P}$ and constant on $T^{F_{0}}$-cosets.

(b) This map is regular and invertible on an open neighborhood of $T_{u n}^{P}$ in $T^{P}$ (with respect to the analytic topology).

(c) $\pi(w k, P, \delta, t)$ is unitary if $t \in T_{u n}^{P}$. 
We can gather all these intertwining operators in a groupoid $\mathcal{W}$, which we define now. The base space of $\mathcal{W}$ is the power set of $F_{0}$ and the collection of arrows from $P$ to $Q$ is

$$
\mathcal{W}_{P Q}:=\left\{w \in W_{0}: w(P)=Q\right\} \times T_{P} \cap T^{P} .
$$

Whenever it is defined, the multiplication in $\mathcal{W}$ is

$$
\left(w_{1}, k_{1}\right) \cdot\left(w_{2}, k_{2}\right)=\left(w_{1} w_{2}, w_{2}^{-1}\left(k_{1}\right) k_{2}\right) .
$$

Families of intertwining operators $\pi(w k, P, \delta, t)$ satisfying the properties listed in Theorem 1.1 are unique only up to normalization by rational functions of $t \in T^{P}$ which are regular in an open neighborhood of $T_{u n}^{P}$, have absolute value equal to 1 on $T_{u n}^{P}$, and are constant on $T^{F_{0}}$-cosets. The intertwining operators defined in Opd1 are normalized in such a way that composition of the intertwining operators corresponds to multiplication of the corresponding elements of $\mathcal{W}$ only up to a scalar.

More precisely, let $g \in \mathcal{W}$ be such that $g w$ is defined. Then there exists a number $\lambda \in \mathbb{C}$ with $|\lambda|=1$, independent of $t$, such that

$$
\pi(g, Q, \sigma, w(k t)) \circ \pi(w k, P, \delta, t)=\lambda \pi(g w k, P, \delta, t)
$$

as rational functions of $t$. We fix such a normalization of the intertwining operators once and for all.

Let $W\left(R_{P}\right) r_{\delta} \in T_{P} / W\left(R_{P}\right)$ be the central character of the $\mathcal{H}_{P}$-representation $\delta$. Then $\left|r_{\delta}\right| \in T_{P, r s}=\exp \left(\mathfrak{a}_{P}\right)$, so we can define

$$
c c_{P}(\delta):=W\left(R_{P}\right) \log \left|r_{\delta}\right| \in \mathfrak{a}_{P} / W\left(R_{P}\right) .
$$

Since the inner product on $\mathfrak{a}$ is $W_{0}$-invariant, the number $\left\|c c_{P}(\sigma)\right\|$ is well-defined.

Theorem 1.2. [Sol3, Theorem 3.3.2]

Let $\rho$ be an irreducible $\mathcal{H}$-representation. There exists a unique association class $\mathcal{W}(P, \delta, t) \in \Xi / \mathcal{W}$ such that the following equivalent properties hold:

(a) $\rho$ is isomorphic to an irreducible quotient of $\pi\left(\xi^{+}\right)$, for some $\xi^{+}=\xi^{+}(\rho) \in$ $\Xi^{+} \cap \mathcal{W}(P, \delta, t)$

(b) $\rho$ is a constituent of $\pi(P, \delta, t)$, and $\left\|c c_{P}(\delta)\right\|$ is maximal for this property.

For any $\xi \in \Xi$ the packet

$$
\operatorname{Irr}_{\mathcal{W} \xi}(\mathcal{H}):=\left\{\rho \in \operatorname{Irr}(\mathcal{H}): \xi^{+}(\rho) \in \mathcal{W} \xi\right\}
$$

is finite, but it is not so easy to predict how many equivalence classes of representations it contains. This is one of the purposes of R-groups.

1.2. The Schwartz algebra. We recall how to complete an affine Hecke algebra to a topological algebra called the Schwartz algebra. As a vector space $\mathcal{S}$ will consist of rapidly decreasing functions on $W$, with respect to some length function. For this purpose it is unsatisfactory that $\ell$ is 0 on the subgroup $Z(W)$, as this can be a large part of $W$. To overcome this inconvenience, let $L: X \otimes \mathbb{R} \rightarrow[0, \infty)$ be a function such that

- $L(X) \subset \mathbb{Z}$

- $L(x+y)=L(x) \quad \forall x \in X \otimes \mathbb{R}, y \in \mathbb{R} R_{0}$,

- $L$ induces a norm on $X \otimes \mathbb{R} / \mathbb{R} R_{0} \cong Z(W) \otimes \mathbb{R}$. 
Now we define

$$
\mathcal{N}(w):=\ell(w)+L(w(0)) \quad w \in W .
$$

Since $Z(W) \oplus \mathbb{Z} R_{0}$ is of finite index in $X$, the set $\{w \in W: \mathcal{N}(w)=0\}$ is finite. Moreover, because $W$ is the semidirect product of a finite group and an abelian group, it is of polynomial growth and different choices of $L$ lead to equivalent length functions $\mathcal{N}$. For $n \in \mathbb{N}$ we define the norm

$$
p_{n}\left(\sum_{w \in W} h_{w} N_{w}\right):=\sup _{w \in W}\left|h_{w}\right|(\mathcal{N}(w)+1)^{n} .
$$

The completion $\mathcal{S}=\mathcal{S}(\mathcal{R}, q)$ of $\mathcal{H}(\mathcal{R}, q)$ with respect to the family of norms $\left\{p_{n}\right\}_{n \in \mathbb{N}}$ is a nuclear Fréchet space. It consists of all possible infinite sums $h=\sum_{w \in W} h_{w} N_{w}$ such that $p_{n}(h)<\infty \forall n \in \mathbb{N}$. By Opd1, Section 6.2] or OpSo2, Appendix A] $\mathcal{S}(\mathcal{R}, q)$ is a unital locally convex *-algebra.

A crucial role in the harmonic analysis on affine Hecke algebra is played by a particular Fourier transform, which is based on the induction data space $\Xi$. Let $\mathcal{V}_{\Xi}$ be the vector bundle over $\Xi$, whose fiber at $(P, \delta, t) \in \Xi$ is the representation space $\mathbb{C}\left[W^{P}\right] \otimes V_{\delta}$ of $\pi(P, \delta, t)$. Let $\operatorname{End}\left(\mathcal{V}_{\Xi}\right)$ be the algebra bundle with fibers $\operatorname{End}_{\mathbb{C}}\left(\mathbb{C}\left[W^{P}\right] \otimes V_{\delta}\right)$. Of course these vector bundles are trivial on every connected component of $\Xi$, but globally not even the dimensions need be constant. Since $\Xi$ has the structure of a complex algebraic variety, we can construct the algebra of polynomial sections of $\operatorname{End}\left(\mathcal{V}_{\Xi}\right)$ :

$$
\mathcal{O}\left(\Xi ; \operatorname{End}\left(\mathcal{V}_{\Xi}\right)\right):=\bigoplus_{P, \delta} \mathcal{O}\left(T^{P}\right) \otimes \operatorname{End}_{\mathbb{C}}\left(\mathbb{C}\left[W^{P}\right] \otimes V_{\delta}\right)
$$

For a submanifold $\Xi^{\prime} \subset \Xi$ we define the algebra $C^{\infty}\left(\Xi^{\prime} ; \operatorname{End}\left(\mathcal{V}_{\Xi}\right)\right)$ in similar fashion. The intertwining operators from Theorem 1.1 give rise to an action of the groupoid $\mathcal{W}$ on the algebra of rational sections of $\operatorname{End}\left(\mathcal{V}_{\Xi}\right)$, by

$$
(w \cdot f)(\xi)=\pi\left(w, w^{-1} \xi\right) f\left(w^{-1} \xi\right) \pi\left(w, w^{-1} \xi\right)^{-1},
$$

whenever $w^{-1} \xi \in \Xi$ is defined. This formula also defines groupoid actions of $\mathcal{W}$ on $C^{\infty}\left(\Xi^{\prime} ; \operatorname{End}\left(\mathcal{V}_{\Xi}\right)\right)$, provided that $\Xi^{\prime}$ is a $\mathcal{W}$-stable submanifold of $\Xi$ on which all the intertwining operators are regular. Given a suitable collection $\Sigma$ of sections of $\left(\Xi^{\prime}, \operatorname{End}\left(\mathcal{V}_{\Xi}\right)\right)$, we write

$\Sigma^{\mathcal{W}}=\left\{f \in \Sigma:(w \cdot f)(\xi)=f(\xi)\right.$ for all $w \in \mathcal{W}, \xi \in \Xi^{\prime}$ such that $w^{-1} \xi$ is defined $\}$.

The Fourier transform for $\mathcal{H}$ is the algebra homomorphism

$$
\begin{aligned}
& \mathcal{F}: \mathcal{H} \rightarrow \mathcal{O}\left(\Xi ; \operatorname{End}\left(\mathcal{V}_{\Xi}\right)\right), \\
& \mathcal{F}(h)(\xi)=\pi(\xi)(h) .
\end{aligned}
$$

The very definition of intertwining operators shows that the image of $\mathcal{F}$ is contained in the algebra $\mathcal{O}\left(\Xi ; \operatorname{End}\left(\mathcal{V}_{\Xi}\right)\right)^{\mathcal{W}}$. By [DeOp1, Theorem 5.3] the Fourier transform extends to an isomorphism of Fréchet *-algebras

$$
\mathcal{F}: \mathcal{S}(\mathcal{R}, q) \rightarrow C^{\infty}\left(\Xi_{\text {un }} ; \operatorname{End}\left(\mathcal{V}_{\Xi}\right)\right)^{\mathcal{W}}
$$

Let $\left(P_{1}, \delta_{1}\right), \ldots,\left(P_{N}, \delta_{N}\right)$ be representatives for the action of $\mathcal{W}$ on pairs $(P, \delta)$. Then the right hand side of (23) can be rewritten as

$$
\bigoplus_{i=1}^{N}\left(C^{\infty}\left(T_{u n}^{P_{i}}\right) \otimes \operatorname{End}\left(\mathbb{C}\left[W^{P_{i}}\right] \otimes V_{\delta_{i}}\right)\right)^{\mathcal{W}_{P_{i}, \delta_{i}}}
$$

where $\mathcal{W}_{P, \delta}=\left\{w \in \mathcal{W}: w(P)=P, \delta \circ \psi_{w}^{-1} \cong \delta\right\}$ is the isotropy group of $(P, \delta)$. 
It was shown in [DeOp1, Corollary 5.5] that (23) implies that

the center $\mathcal{Z}$ of $\mathcal{S}$ is isomorphic to $C^{\infty}\left(\Xi_{u n}\right)^{\mathcal{W}}$,

so the space of central characters of $\mathcal{S}$ is $\Xi_{u n} / \mathcal{W}$. We let $\operatorname{Mod}_{f}(\mathcal{S})$ be the category of finite dimensional $\mathcal{S}$-modules and $\operatorname{Mod}_{f, \mathcal{W} \xi}(\mathcal{S})$ the full subcategory of modules which admit the central character $\mathcal{W} \xi$. The collection $\operatorname{Irr}_{\mathcal{W} \xi}(\mathcal{S})$ of (equivalence classes of) irreducible objects in $\operatorname{Mod}_{f, \mathcal{W} \xi}(\mathcal{S})$ equals $\operatorname{Irr}_{\mathcal{W} \xi}(\mathcal{H})$, in the notation of (20).

\section{Formal completion of the Schwartz Algebra}

We study how the Ext-groups of Fréchet $\mathcal{S}$-modules behave under formal completion with respect to a maximal ideal of the center. For compatibility with OpSo1] we will work in the category $\operatorname{Mod}_{b o r}(\mathcal{S})$ of complete bornological modules over the nuclear Fréchet algebra $\mathcal{S}$, equipped with its precompact bornology. This is an exact category with respect to the class of $\mathcal{S}$-module extensions that are split as bornological vector spaces.

Two remarks for readers who are not familiar with bornologies. As discussed in the introduction, one may think of Fréchet $\mathcal{S}$-modules and exact sequences which admit a continuous linear splitting, that is just as good in the setting of this paper. Moreover, if one is only interested in finite dimensional modules, then the subtleties with topological modules are superfluous and all the results can also be obtained in an algebraic way. However, the equivalence of the algebraic and the bornological approach in a finite dimensional setting is not automatic, it follows from the existence of certain nice projective resolutions OpSo1.

Although the entire section is formulated in terms of the Schwartz algebra $\mathcal{S}$, the essence is the study of algebras of smooth functions on manifolds. The results and proofs are equally valid, and of some independent interest, if one replaces $\mathcal{S}$ by $C^{\infty}(M)$, where $M$ is a smooth manifold such that $C^{\infty}(M) \cong \mathcal{S}(\mathbb{Z})$ as Fréchet spaces. According to [MeVo, Satz 31.16] this is the case when $M$ has no boundary or is the closure of an open bounded subset of $\mathbb{R}^{n}$ with $C^{1}$ boundary. The formal completion of $C^{\infty}(M)$ with respect to a maximal ideal is a power series ring, which provides a good geometric interpretation for the material in this section.

In (25) $\mathcal{Z} \cong C^{\infty}\left(\Xi_{u n}\right)^{\mathcal{W}}$ is equipped with the Fréchet topology from $C^{\infty}\left(\Xi_{u n}\right)$. We fix $\xi=(P, \delta, t) \in \Xi_{u n}$. Let $m_{\mathcal{W} \xi}^{\infty}$ denote the closed ideal of $\mathcal{Z}$ of functions that are flat at $\mathcal{W} \xi$, that is, their Taylor series around any point of $\mathcal{W} \xi$ is zero. We define a Fréchet module $\widehat{\mathcal{Z}}_{\mathcal{W} \xi}$ over $\mathcal{Z}$ by the exact sequence

$$
0 \rightarrow m_{\mathcal{W} \xi}^{\infty} \rightarrow \mathcal{Z} \rightarrow \widehat{\mathcal{Z}}_{\mathcal{W} \xi} \rightarrow 0
$$

It follows easily from Borel's lemma that the Taylor series map $\tau_{\xi}$ defines a continuous surjection $\tau_{\xi}: \mathcal{Z} \rightarrow \mathcal{F}_{\xi}^{\mathcal{W}_{\xi}}$ where $\mathcal{F}_{\xi}^{\mathcal{W}_{\xi}}$ denotes the unital Fréchet algebra of $\mathcal{W}_{\xi}$-invariant formal power series at $\xi$. According to [Tou, Remarque IV.3.9] $\tau_{\xi}$ is not linearly split. The kernel of $\tau_{\xi}$ is isomorphic to $m_{\mathcal{W} \xi}^{\infty}$, hence we may identify $\widehat{\mathcal{Z}}_{\mathcal{W} \xi}$ and $\mathcal{F}_{\xi}^{\mathcal{W}} \mathcal{W}_{\xi}$. Notice that this algebra is Noetherian, like any power series ring with finitely many variables and coefficients in a field. By Whitney's spectral theorem [Tou, V.1.6]

$$
\overline{\left(m_{\mathcal{W} \xi}^{\infty}\right)^{2}}=\overline{m_{\mathcal{W} \xi}^{\infty}}=m_{\mathcal{W} \xi}^{\infty} .
$$


For similar reasons $\mathcal{I}_{\mathcal{W} \xi}:=m_{\mathcal{W} \xi}^{\infty} \mathcal{S}$ is a closed two-sided ideal in $\mathcal{S}$. Hence by applying the completed projective tensor product functor ? $\widehat{\otimes}_{\mathcal{Z}} \mathcal{S}$ to (26) we obtain

$$
0 \rightarrow \mathcal{I}_{\mathcal{W} \xi} \rightarrow \mathcal{S} \rightarrow \widehat{\mathcal{Z}}_{\mathcal{W} \xi} \widehat{\otimes}_{\mathcal{Z}} \mathcal{S} \rightarrow 0 .
$$

We define the completion of $\mathcal{S}$ at $\mathcal{W} \xi$ to be the Fréchet $\widehat{\mathcal{Z}}_{\mathcal{W} \xi}$-algebra

$$
\widehat{\mathcal{S}}_{\mathcal{W} \xi}:=\widehat{\mathcal{Z}}_{\mathcal{W} \xi} \widehat{\otimes}_{\mathcal{Z}} \mathcal{S} \text {. }
$$

By (23) and (25) $\mathcal{S}$ is a finitely generated $\mathcal{Z}$-module, so $\widehat{\mathcal{S}}_{\mathcal{W} \xi}$ is left and right Noetherian. In particular the category $\operatorname{Mod}_{f g}\left(\widehat{\mathcal{S}}_{\mathcal{W} \xi}\right)$ of finitely generated $\widehat{S}_{\mathcal{W} \xi}$-modules is closed under extensions. Any $M \in \operatorname{Mod}_{f g}\left(\widehat{\mathcal{S}}_{\mathcal{W} \xi}\right)$ admits a finite presentation

$$
\widehat{\mathcal{S}}_{\mathcal{W} \xi}^{n_{2}} \rightarrow \widehat{\mathcal{S}}_{\mathcal{W} \xi}^{n_{1}} \rightarrow M \rightarrow 0 .
$$

It follows that $\widehat{\mathcal{S}}_{\mathcal{W} \xi}$ is a Fréchet space of finite type, i.e. that its topology is defined by an increasing sequence of seminorms all of whose cokernels have finite codimension. By Kopp all continuous linear maps between such Fréchet spaces have closed images. In particular, the relations module $\operatorname{im}\left(\widehat{\mathcal{S}}_{\mathcal{W} \xi}^{n_{2}} \rightarrow \widehat{\mathcal{S}}_{\mathcal{W} \xi}^{n_{1}}\right)$ in (30) is closed, showing that $M$ is a Fréchet module (i.e. the canonical topology on $M$ in the sense of [Tou] is separated, hence defines a Fréchet module structure on $M$ ). It also follows easily that morphisms between finitely generated $\widehat{\mathcal{S}}_{\mathcal{W} \xi}$-modules are automatically continuous with respect to the canonical topology. Summarizing:

Lemma 2.1. $\operatorname{Mod}_{f g}\left(\widehat{\mathcal{S}}_{\mathcal{W} \xi}\right)$ is a Serre subcategory of $\operatorname{Mod}_{\text {Fré }}\left(\widehat{\mathcal{S}}_{\mathcal{W} \xi}\right)$, the category of Fréchet $\widehat{\mathcal{S}}_{\mathcal{W} \xi}$-modules.

Let $q: \mathcal{S} \rightarrow \widehat{\mathcal{S}}_{\mathcal{W} \xi}$ be the quotient map and

$$
q^{*}: \operatorname{Mod}_{\text {bor }}\left(\widehat{\mathcal{S}}_{\mathcal{W} \xi}\right) \rightarrow \operatorname{Mod}_{\text {bor }}(\mathcal{S})
$$

the associated pullback functor. In the opposite direction the completed bornological tensor product provides a functor

$$
\widehat{\mathcal{S}}_{\mathcal{W} \xi} \widehat{\otimes}_{\mathcal{S}}: \operatorname{Mod}_{\text {bor }}(\mathcal{S}) \rightarrow \operatorname{Mod}_{\text {bor }}\left(\widehat{\mathcal{S}}_{\mathcal{W} \xi}\right) .
$$

We remark that for Fréchet modules this tensor product agrees with the completed projective one.

Lemma 2.2. Let $V \in \operatorname{Mod}_{\text {bor }}(\mathcal{S})$ and abbreviate $\widehat{V}_{\mathcal{W} \xi}:=\widehat{\mathcal{S}}_{\mathcal{W} \xi} \widehat{\otimes}_{\mathcal{S}} V$.

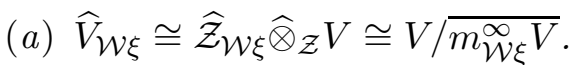

(b) For all $M \in \operatorname{Mod}_{\text {bor }}\left(\widehat{\mathcal{S}}_{\mathcal{W} \xi}\right)$ there is a natural isomorphism $\widehat{\mathcal{S}}_{\mathcal{W} \xi} \widehat{\otimes}_{\mathcal{S}} q^{*} M \cong M$.

(c) $q^{*}$ is fully faithful and exact.

(d) $q^{*}$ is right adjoint to $\widehat{\mathcal{S}}_{\mathcal{W} \xi} \widehat{\otimes}_{\mathcal{S}}$.

(e) $\operatorname{Mod}_{\text {bor }}^{\mathcal{W} \xi \text { tor }}(\mathcal{S}):=\left\{M \in \operatorname{Mod}_{\text {bor }}(\mathcal{S}) \mid m_{\mathcal{W} \xi}^{\infty} M=0\right\}$ is a Serre subcategory of $\operatorname{Mod}_{b o r}(\mathcal{S})$.

$(f) q^{*}$ defines equivalences of exact categories $\operatorname{Mod}_{\text {bor }}\left(\widehat{\mathcal{S}}_{\mathcal{W} \xi}\right) \rightarrow \operatorname{Mod}_{\text {bor }}^{\mathcal{W} \xi \text {,tor }}(\mathcal{S})$ and $\operatorname{Mod}_{\text {Fré }}\left(\widehat{\mathcal{S}}_{\mathcal{W} \xi}\right) \rightarrow \operatorname{Mod}_{\text {Fré }}^{\mathcal{W} \xi \text { tor }}(\mathcal{S})$.

Proof. (a) By the associativity of bornological tensor products

$$
\begin{aligned}
\widehat{V}_{\mathcal{W} \xi}=\widehat{\mathcal{S}}_{\mathcal{W} \xi} \widehat{\otimes}_{\mathcal{S}} V= & \widehat{\mathcal{Z}}_{\mathcal{W} \xi} \widehat{\otimes}_{\mathcal{Z}} \mathcal{S} \widehat{\otimes}_{\mathcal{S}} V \cong \widehat{\mathcal{Z}}_{\mathcal{W} \xi} \widehat{\otimes}_{\mathcal{Z}} V \\
& \cong \widehat{\mathcal{Z}}_{\mathcal{W} \xi} \widehat{\otimes}_{\mathcal{Z}}\left(V / m_{\mathcal{W} \xi}^{\infty} V\right) \cong \widehat{\mathcal{Z}}_{\mathcal{W} \xi} \widehat{\otimes}_{\widehat{\mathcal{Z}}_{\mathcal{W} \xi}}\left(V / m_{\mathcal{W} \xi}^{\infty} V\right) \cong V / m_{\mathcal{W} \xi}^{\infty} V
\end{aligned}
$$


(b) follows immediately from (a)

(c) is obvious.

(d) Since the kernel of any $\mathcal{S}$-module homomorphism $V \rightarrow q^{*} M$ contains $\overline{m_{\mathcal{W} \xi}^{\infty} V}$,

$$
\operatorname{Hom}_{\mathcal{S}}\left(V, q^{*} M\right) \cong \operatorname{Hom}_{\mathcal{S}}\left(V / \overline{m_{\mathcal{W} \xi}^{\infty} V}, q^{*} M\right) .
$$

By (a), (b) and (c) the right hand side is isomorphic to

$$
\operatorname{Hom}_{\mathcal{S}}\left(q^{*} \widehat{V}_{\mathcal{W} \xi}, q^{*} M\right) \cong \operatorname{Hom}_{\widehat{\mathcal{S}}_{\mathcal{W} \xi}}\left(\widehat{V}_{\mathcal{W} \xi}, M\right)
$$

(e) The only nontrivial part concerns extensions. Let

$$
0 \rightarrow V_{1} \stackrel{i}{\rightarrow} V_{2} \stackrel{p}{\rightarrow} V_{3} \rightarrow 0
$$

be a short exact sequence in $\operatorname{Mod}_{\text {bor }}(\mathcal{S})$ and assume that $m_{\mathcal{W} \xi}^{\infty} V_{1}=m_{\mathcal{W} \xi}^{\infty} V_{3}=0$. For $m \in m_{\mathcal{W} \xi}^{\infty} V_{2}$ and $v_{2} \in V_{2}$ we have $p\left(m v_{2}\right)=m p\left(v_{2}\right) \in m_{\mathcal{W} \xi}^{\infty} V_{3}=0$, so $m_{\mathcal{W} \xi}^{\infty} V_{2} \subset$ $i\left(V_{1}\right)$. Moreover by (27)

$$
m_{\mathcal{W} \xi}^{\infty} V_{2}=\overline{\left(m_{\mathcal{W} \xi}^{\infty}\right)^{2}} V_{2} \subset \overline{m_{\mathcal{W} \xi}^{\infty} i\left(m_{\mathcal{W} \xi}^{\infty} V_{1}\right)}=\overline{m_{\mathcal{W} \xi}^{\infty} i(0)}=0
$$

so $V_{2} \in \operatorname{Tor}_{\mathcal{W} \xi}^{\infty}(\mathcal{S})$.

(f) In view of (c) we only have to show that $q^{*}: \operatorname{Mod}_{b o r}\left(\widehat{\mathcal{S}}_{\mathcal{W} \xi}\right) \rightarrow \operatorname{Mod}_{b o r}^{\mathcal{W} \xi \text {,tor }}(\mathcal{S})$ is essentially bijective. Clearly any $V \in \operatorname{Mod}_{b o r}^{\mathcal{V} \xi, t o r}(\mathcal{S})$ can be considered as a $\widehat{S}_{\mathcal{W} \xi^{-m o d u l e}} V^{\prime}$, and as such $V=q^{*} V^{\prime}$. Conversely, if $V \cong q^{*} M$ for some $M \in$ $\operatorname{Mod}_{b o r}^{\mathcal{W} \xi, t o r}\left(\widehat{\mathcal{S}}_{\mathcal{W} \xi}\right)$, then

$$
M \cong \widehat{\mathcal{S}}_{\mathcal{W} \xi} \widehat{\otimes}_{\mathcal{S}} q^{*} M \cong \widehat{\mathcal{S}}_{\mathcal{W} \xi} \widehat{\otimes}_{\mathcal{S}} V=\widehat{V}_{\mathcal{W} \xi}
$$

by part (b).

The spaces $\operatorname{Ext}_{\mathcal{S}}^{n}\left(\pi, \pi^{\prime}\right)$ carry a natural $\mathcal{Z}$-module structure. Let the tempered central characters of $\pi$ and $\pi^{\prime}$ be $\mathcal{W} \xi$ and $\mathcal{W} \xi^{\prime}$ respectively. Standard arguments show that $\operatorname{Ext}_{\mathcal{S}}^{n}\left(\pi, \pi^{\prime}\right)$ has central character $\mathcal{W} \xi$ if $\mathcal{W} \xi=\mathcal{W} \xi^{\prime}$, and $\operatorname{Ext}_{\mathcal{S}}^{n}\left(\pi, \pi^{\prime}\right)=0$ otherwise. To compute these Ext-groups we shall at some point want to pass to the formal completion of $\mathcal{S}$ at $\mathcal{W} \xi$. For that purpose we would like the functor $\widehat{\mathcal{S}}_{\mathcal{W} \xi} \widehat{\otimes}_{\mathcal{S}}$ to be exact, but unfortunately the authors do not know whether it is so on the categories of Fréchet $\mathcal{S}$-modules or bornological $\mathcal{S}$-modules. Moreover this functor does not always respect linear splittings of short exact sequences.

Therefore we restrict to smaller module categories. Let $\mathcal{S}(\mathbb{Z})$ be the nuclear Fréchet space of rapidly decreasing functions $\mathbb{Z} \rightarrow \mathbb{C}$ and let $\Omega$ (resp. $D N \Omega$ ) be the category of Fréchet spaces that are isomorphic to a quotient (resp. a direct summand) of $\mathcal{S}(\mathbb{Z})$. Our notation is motivated by certain properties $(\Omega)$ and $(D N)$ of Fréchet spaces, which characterize the quotients and the subspaces of $\mathcal{S}(\mathbb{Z})$ among all nuclear Fréchet spaces Vogt].

These categories are exact but not abelian, because some morphisms do not have a kernel or a cokernel. They are suitable to overcome to problems that can arise from closed subspaces which are not complemented:

Theorem 2.3. (a) The category $\Omega$ is closed under extensions of Fréchet spaces.

(b) Let $0 \rightarrow V_{1} \rightarrow V_{2} \rightarrow V_{3} \rightarrow 0$ be a short exact sequence of Fréchet spaces. It splits whenever $V_{1}$ is a quotient of $\mathcal{S}(\mathbb{Z})$ and $V_{3}$ is a subspace of $\mathcal{S}(\mathbb{Z})$.

(c) Every object of $D N \Omega$ is projective in $\Omega$. 
Proof. (a) is [VoWa, Lemma 1.7].

(b) is Vogt, Theorem 5.1].

(c) follows easily from (b), as in [Vogt, Theorem 1.8].

The very definition 21] shows that $\mathcal{S} \cong \mathcal{S}(\mathbb{Z})$ as Fréchet spaces, and hence $\widehat{\mathcal{S}}_{\mathcal{W} \xi} \in \Omega$. However, $\widehat{\mathcal{S}}_{\mathcal{W} \xi}$ is not isomorphic to a subspace of $\mathcal{S}(\mathbb{Z})$, because it is a finite extension of a Fréchet algebra of formal power series.

Let $\operatorname{Mod}_{\Omega}(\mathcal{S})$ be the full subcategory of $\operatorname{Mod}_{\text {Fré }}(\mathcal{S})$ consisting of modules whose underlying spaces belong to $\Omega$. We define $\operatorname{Mod}_{\Omega}(\mathcal{Z}), \operatorname{Mod}_{\Omega}\left(\widehat{\mathcal{S}}_{\mathcal{W} \xi}\right)$ and $\operatorname{Mod}_{D N \Omega}(\mathcal{S})$ similarly. In these categories the morphisms are all continuous module homomorphisms. Apart from $\operatorname{Mod}_{D N \Omega}(\mathcal{S})$ they do not have enough projective objects, so derived functors are not defined for all modules. Nevertheless the Yoneda Ext-groups, constructed as equivalence classes of higher extensions, are always available.

Lemma 2.4. The categories $\operatorname{Mod}_{D N \Omega}(\mathcal{S}), \operatorname{Mod}_{\Omega}(\mathcal{S})$ and $\operatorname{Mod}_{\Omega}\left(\widehat{\mathcal{S}}_{\mathcal{W} \xi}\right)$ have the following properties:

(a) They are closed under extensions of Fréchet modules.

(b) Every short exact sequence in $\operatorname{Mod}_{D N \Omega}(\mathcal{S})$ admits a continuous linear splitting.

(c) They are exact categories in the sense of Quillen if we declare all short exact sequences to be admissible.

(d) For $F \in D N \Omega$ the $\mathcal{S}$-module $\mathcal{S} \widehat{\otimes} F$ is projective in $\operatorname{Mod}_{D N \Omega}(\mathcal{S})$, in $\operatorname{Mod}_{\Omega}(\mathcal{S})$ and in $\operatorname{Mod}_{\text {bor }}(\mathcal{S})$, while $\widehat{\mathcal{S}}_{\mathcal{W} \xi} \widehat{\otimes} F$ is projective in $\operatorname{Mod}_{\Omega}\left(\widehat{\mathcal{S}}_{\mathcal{W} \xi}\right)$.

(e) $\operatorname{Mod}_{D N \Omega}(\mathcal{S})$ and $\operatorname{Mod}_{f g}\left(\widehat{\mathcal{S}}_{\mathcal{W} \xi}\right)$ have enough projectives.

$(f)$ The following embeddings preserve Yoneda Ext-groups: $\operatorname{Mod}_{D N \Omega}(\mathcal{S}) \rightarrow \operatorname{Mod}_{\text {bor }}(\mathcal{S})$, $\operatorname{Mod}_{D N \Omega}(\mathcal{S}) \rightarrow \operatorname{Mod}_{\Omega}(\mathcal{S}), \operatorname{Mod}_{f g}\left(\widehat{\mathcal{S}}_{\mathcal{W} \xi}\right) \rightarrow \operatorname{Mod}_{\Omega}\left(\widehat{\mathcal{S}}_{\mathcal{W} \xi}\right)$ and $\operatorname{Mod}_{f g}\left(\widehat{\mathcal{S}}_{\mathcal{W} \xi}\right) \rightarrow$ $\operatorname{Mod}\left(\widehat{\mathcal{S}}_{\mathcal{W} \xi}\right)$.

Proof. (a) follows from Theorem 2.3 .

(b) follows from Theorem 2.3. b.

(c) The definition of an exact category stems from Qui, Section 2], while it is worked out in [Kel, Appendix A] which axioms are really necessary. All of these are trivially satisfied, except for the pullback and pushout properties. Since the verification of these properties is the same in all four categories, we only write it down in $\operatorname{Mod}_{\Omega}(\mathcal{S})$.

Suppose that $0 \rightarrow V_{1} \stackrel{i}{\rightarrow} V_{2} \stackrel{p}{\rightarrow} V_{3} \rightarrow 0$ is admissible exact in $\operatorname{Mod}_{\Omega}(\mathcal{S})$ and that $f: V_{1} \rightarrow M$ is any morphism in $\operatorname{Mod}_{\Omega}(\mathcal{S})$. We have to show that there is a pushout diagram

$$
\begin{array}{lllll}
V_{1} & \stackrel{i}{\rightarrow} & V_{2} & \stackrel{p}{\rightarrow} & V_{3} \\
\downarrow f & & \downarrow & & \\
M & \stackrel{g}{\rightarrow} & P & \rightarrow & P / g(M)
\end{array}
$$

in $\operatorname{Mod}_{\Omega}(\mathcal{S})$, with an admissible exact second row. Since $i$ is injective the image of $(i,-f): V_{1} \rightarrow V_{2} \oplus M$ is closed. Hence $P:=V_{2} \oplus M / \mathrm{im}(i,-f)$ is a Fréchet $\mathcal{S}$-module. The canonical map $g: M \rightarrow P$ is injective and

$$
P / g(M) \cong \frac{V_{2} \oplus M}{g(M)+\operatorname{im}(i,-f)} \cong V_{2} / i\left(V_{1}\right) \cong V_{3},
$$

which belongs to $\operatorname{Mod}_{\Omega}(\mathcal{S})$. By part (a) $P \in \operatorname{Mod}_{\Omega}(\mathcal{S})$, so $0 \rightarrow M \stackrel{g}{\rightarrow} P \rightarrow$ $P / g(M) \rightarrow 0$ is admissible. 
Concerning pullbacks, let $h: N \rightarrow V_{3}$ be any morphism in $\operatorname{Mod}_{\Omega}(\mathcal{S})$. We have to show that there is a pullback diagram

$$
\begin{array}{rrrrr}
\operatorname{ker}(Q \rightarrow N) & \rightarrow & Q & \rightarrow & N \\
& & \downarrow & & \downarrow h \\
V_{1} & \stackrel{i}{\rightarrow} & V_{2} & \stackrel{p}{\rightarrow} & V_{3}
\end{array}
$$

in $\operatorname{Mod}_{\Omega}(\mathcal{S})$, with an admissible exact first row. Let $Q$ be the Fréchet $\mathcal{S}$-module $\left\{(v, n) \in V_{2} \oplus N \mid p(v)=h(n)\right\}=\operatorname{ker}\left(p-h: V_{2} \oplus N \rightarrow V_{3}\right)$. Then

$$
\operatorname{ker}(Q \rightarrow N) \cong \operatorname{ker} p \cong V_{1} \in \operatorname{Mod}_{D N \Omega}(\mathcal{S}),
$$

so $Q \in \operatorname{Mod}_{\Omega}(\mathcal{S})$ by part (a).

(d) For every $M \in \operatorname{Mod}_{\Omega}\left(\widehat{\mathcal{S}}_{\mathcal{W} \xi}\right)$ there is an isomorphism

$$
\operatorname{Hom}_{\operatorname{Mod}_{\Omega}\left(\widehat{\mathcal{S}}_{\mathcal{W} \xi}\right)}\left(\widehat{\mathcal{S}}_{\mathcal{W} \xi} \widehat{\otimes} F, M\right) \cong \operatorname{Hom}_{F r e ́}(F, M) .
$$

By Theorem 2.3.c the right hand side is exact as a functor of $M \in \Omega$, so $\widehat{\mathcal{S}}_{\mathcal{W} \xi} \widehat{\otimes} V$ is projective in $\operatorname{Mod}_{\Omega}\left(\widehat{\mathcal{S}}_{\mathcal{W} \xi}\right)$. The same proof applies to $\mathcal{S} \widehat{\otimes} F$ as an object of $\operatorname{Mod}_{D N \Omega}(\mathcal{S})$ and of $\operatorname{Mod}_{\Omega}(\mathcal{S}) . \operatorname{In} \operatorname{Mod}_{b o r}(\mathcal{S})$ exact sequences are required to have linear splittings, which implies that topologically free modules are projective.

(e) For any $(\pi, V) \in \operatorname{Mod}_{D N \Omega}(\mathcal{S})$ we have the canonical $\mathcal{S}$-module surjection

$$
\mathcal{S} \widehat{\otimes} V \rightarrow V: s \otimes v \mapsto \pi(s) v,
$$

which is linearly split by $v \mapsto 1 \otimes v$. Its kernel $N$ is (as Fréchet space) a direct summand of $\mathcal{S} \widehat{\otimes} V$, which in turn is a direct summand of $\mathcal{S} \widehat{\otimes} \mathcal{S}(\mathbb{Z})$. Thus $N \in$ $\operatorname{Mod}_{D N \Omega}(\mathcal{S})$ and we can build a projective resolution

$$
0 \leftarrow V \leftarrow \mathcal{S} \widehat{\otimes} V \leftarrow \mathcal{S} \widehat{\otimes} N \leftarrow \cdots
$$

Let $M$ be a finitely generated Fréchet $\widehat{\mathcal{S}}_{\mathcal{W} \xi}$-module, say it is a quotient of $\widehat{\mathcal{S}}_{\mathcal{W} \xi}^{n}$. Since $\widehat{\mathcal{S}}_{\mathcal{W} \xi}$ is Noetherian, every submodule of a finitely generated $\widehat{\mathcal{S}}_{\mathcal{W} \xi \text {-module is }}$ again finitely generated. This applies in particular to the kernel of the quotient map $\widehat{\mathcal{S}}_{\mathcal{W} \xi}^{n} \rightarrow M$, so we can construct a projective resolution with free $\widehat{\mathcal{S}}_{\mathcal{W} \xi}$-modules of finite rank.

(f) By part (d) and Lemma 2.1, the projective resolutions from part (e) remain projective in $\operatorname{Mod}_{b o r}(\mathcal{S})$ and in $\operatorname{Mod}_{\Omega}(\mathcal{S})$, respectively in $\operatorname{Mod}_{\Omega}\left(\widehat{\mathcal{S}}_{\mathcal{W} \xi}\right)$ and in $\operatorname{Mod}\left(\widehat{\mathcal{S}}_{\mathcal{W} \xi}\right)$. Furthermore part (b) guarantees that such a resolution of $\mathcal{S}$-modules admits a continuous linear splitting, so it is admissible as a resolution of bornological modules. It is well-known that the Yoneda Ext-groups in an exact category agree with the derived functors of Hom when both are defined, see for example [Mac, Theorem $6.4]$.

\subsection{Exactness of formal completion.}

Theorem 2.5. The functor $\widehat{S}_{\mathcal{W} \xi} \widehat{\otimes}_{\mathcal{S}}: \operatorname{Mod}_{\Omega}(\mathcal{S}) \rightarrow \operatorname{Mod}_{\Omega}\left(\widehat{S}_{\mathcal{W} \xi}\right)$ is exact.

Proof. We have to show that the image of any short exact sequence under this functor is again a short exact sequence. Since $\widehat{\mathcal{S}}_{\mathcal{W} \xi} \widehat{\otimes}_{\mathcal{S}} V \cong \widehat{\mathcal{Z}}_{\mathcal{W} \xi} \widehat{\otimes}_{\mathcal{Z}} V$ for all $V \in$ $\operatorname{Mod}_{\Omega}(\mathcal{S}), \quad \widehat{S}_{\mathcal{W} \xi} \widehat{\otimes}_{\mathcal{S}}$ is exact if and only if $\operatorname{Tor}_{1}^{\operatorname{Mod}(\mathcal{Z})}\left(\widehat{\mathcal{Z}}_{\mathcal{W} \xi}, V\right)=0$ for all $V \in$ $\operatorname{Mod}_{\Omega}(\mathcal{S})$. Because $\mathcal{S}$ is finitely generated as a $\mathcal{Z}$-module and $V \in \Omega$, there exists a short exact sequence of Fréchet $\mathcal{Z}$-modules

$$
0 \rightarrow R \rightarrow \mathcal{Z} \widehat{\otimes} \mathcal{S}(\mathbb{Z}) \rightarrow V \rightarrow 0 .
$$


The analogue of Lemma 2.4.d for $\mathcal{Z}$ shows that $\mathcal{Z} \widehat{\otimes} \mathcal{S}(\mathbb{Z})$ is projective in $\operatorname{Mod}_{\Omega}(\mathcal{Z})$. By the definition of the torsion functor and by Lemma 2.2. a

$$
\begin{aligned}
\operatorname{Tor}_{1}^{\operatorname{Mod}_{\Omega}(\mathcal{Z})}\left(\widehat{\mathcal{Z}}_{\mathcal{W} \xi}, V\right)=\operatorname{ker}\left(\widehat{\mathcal{Z}}_{\mathcal{W} \xi} \widehat{\otimes}_{\mathcal{Z}} R \rightarrow \widehat{\mathcal{Z}}_{\mathcal{W} \xi} \widehat{\otimes} \mathcal{S}(\mathbb{Z})\right) \cong \\
\operatorname{ker}\left(\frac{R}{\overline{m_{\mathcal{W} \xi}^{\infty} R}} \rightarrow \frac{\mathcal{Z} \widehat{\otimes} \mathcal{S}(\mathbb{Z})}{m_{\mathcal{W} \xi}^{\infty} \widehat{\otimes} \mathcal{S}(\mathbb{Z})}\right) \cong \frac{\left(m_{\mathcal{W} \xi}^{\infty} \widehat{\otimes} \mathcal{S}(\mathbb{Z})\right) \cap R}{\overline{m_{\mathcal{W} \xi}^{\infty} R}} .
\end{aligned}
$$

Thus we have to show that

$$
\left(m_{\mathcal{W} \xi}^{\infty} \widehat{\otimes} \mathcal{S}(\mathbb{Z})\right) \cap R \subset \overline{m_{\mathcal{W} \xi}^{\infty} R},
$$

which we will do with a variation on [ToMe, Chapitre 1]. Via the Fourier transform $\mathcal{S}(\mathbb{Z})$ is isomorphic to $C^{\infty}\left(S^{1}\right)$, so

$$
\mathcal{Z} \widehat{\otimes} \mathcal{S}(\mathbb{Z}) \cong C^{\infty}\left(\Xi_{\text {un }}\right)^{\mathcal{W}} \widehat{\otimes} C^{\infty}\left(S^{1}\right) \cong C^{\infty}\left(\Xi_{\text {un }} \times S^{1}\right)^{\mathcal{W}} .
$$

Under this isomorphism $m_{\mathcal{W} \xi}^{\infty} \widehat{\otimes} \mathcal{S}(\mathbb{Z})$ corresponds to the closed ideal $m_{\mathcal{W} \xi \times S^{1}}^{\infty} \subset$ $C^{\infty}\left(\Xi_{\text {un }} \times S^{1}\right)^{\mathcal{W}}$ of functions that are flat on $\mathcal{W} \xi \times S^{1}$.

Let $\phi \in\left(m_{\mathcal{W} \xi}^{\infty} \widehat{\otimes} \mathcal{S}(\mathbb{Z})\right) \cap R$. Tougeron and Merrien [ToMe, p. 183-185] construct a function $\psi \in m_{\mathcal{W} \xi \times S^{1}}^{\infty}$ which is strictly positive outside $\mathcal{W} \xi \times S^{1}$ and divides $\phi$ in $C^{\infty}\left(\Xi_{u n} \times S^{1}\right)^{\mathcal{W}}$. We take a closer look at this construction. The first thing to note is that ToMe works with smooth functions on manifolds, not on orbifolds like $\Xi_{\text {un }} / \mathcal{W} \times S^{1}$. But this is only a trifle, for we can construct a suitable $\tilde{\psi} \in$ $C^{\infty}\left(\Xi_{\text {un }} \times S^{1}\right)$ and average it over $\mathcal{W}$. Secondly we observe that, because $S^{1}$ is compact, we can take all steps from [ToMe] with $S^{1}$-invariant functions on $\Xi_{\text {un }} \times S^{1}$. Thus we obtain a series of $\mathcal{W} \times S^{1}$-invariant functions $\sum_{i} \epsilon_{i}$ which converges to a $\psi$ as above. (Compared to [ToMe] we include a suitable power of 1/2 in $\epsilon_{i}$.) Moreover the support of $\epsilon_{i}$ is disjoint from $\mathcal{W} \xi \times S^{1}$, so

$$
\epsilon_{i} / \psi \in m_{\mathcal{W} \xi}^{\infty} \subset C^{\infty}\left(\Xi_{u n}\right)^{\mathcal{W}} \subset C^{\infty}\left(\Xi_{u n} \times S^{1}\right) .
$$

Since $R$ is a $\mathcal{Z}$-submodule of $C^{\infty}\left(\Xi_{\text {un }} \times S^{1}\right)^{\mathcal{W}}, \phi \epsilon_{i} / \psi \in m_{\mathcal{W} \xi}^{\infty} R$ for all $i$. Hence

$$
\phi=\psi \phi / \psi=\sum_{i} \phi \epsilon_{i} / \psi \in \overline{m_{\mathcal{W} \xi}^{\infty} R}
$$

which proves (32).

Corollary 2.6. Let $M, N \in \operatorname{Mod}_{\Omega}\left(\widehat{\mathcal{S}}_{\mathcal{W} \xi}\right)$ and let $n \in \mathbb{Z}_{\geq 0}$.

(a) There is an isomorphism of $\widehat{\mathcal{Z}}_{\mathcal{W} \xi}$-modules

$$
\operatorname{Ext}_{\operatorname{Mod}_{\Omega}(\mathcal{S})}^{n}\left(q^{*} N, q^{*} M\right) \cong \operatorname{Ext}_{\operatorname{Mod}_{\Omega}\left(\widehat{\mathcal{S}}_{\mathcal{W} \xi}\right)}^{n}(N, M) .
$$

(b) Let $V \in \operatorname{Mod}_{D N \Omega}(\mathcal{S})$ be such that $\widehat{V}_{\mathcal{W} \xi}$ is finitely generated over $\widehat{\mathcal{S}}_{\mathcal{W} \xi}$. There are isomorphisms of $\widehat{\mathcal{Z}}_{\mathcal{W} \xi}$-modules

$$
\operatorname{Ext}_{\operatorname{Mod}_{b o r}(\mathcal{H})}^{n}\left(V, q^{*} M\right) \cong \operatorname{Ext}_{\operatorname{Mod}_{b o r}(\mathcal{S})}^{n}\left(V, q^{*} M\right) \cong \operatorname{Ext}_{\widehat{\mathcal{S}}_{\mathcal{W} \xi}}^{n}\left(\widehat{V}_{\mathcal{W} \xi}, M\right) .
$$

(c) In case $V$ has finite dimension the groups from (b) are also isomorphic to $\operatorname{Ext}_{\mathcal{H}}^{n}\left(V, q^{*} M\right)$ and $\operatorname{Ext}_{\mathcal{S}}^{n}\left(V, q^{*} M\right)$, that is, to the Ext-groups defined in the category of all (algebraic) $\mathcal{H}$-or $\mathcal{S}$-modules. 
Proof. The $\widehat{\mathcal{Z}}_{\mathcal{W} \xi}$-module structure is defined via

$$
\widehat{\mathcal{Z}}_{\mathcal{W} \xi} \rightarrow \operatorname{End}_{\mathcal{H}}\left(q^{*} M\right)=\operatorname{End}_{\mathcal{S}}\left(q^{*} M\right)=\operatorname{End}_{\widehat{\mathcal{S}}_{\mathcal{W} \xi}}(M)
$$

and right multiplication of $\operatorname{Ext}_{A}^{n}(?, M)$ with $\operatorname{Ext}_{A}^{0}(M, M)$. The asserted isomorphisms preserve this additional structure because in the below proof $M$ is kept constant.

(a) Consider the endofunctor $E$ of $\operatorname{Mod}_{\Omega}(\mathcal{S})$ defined by $E(V)=q^{*}\left(\widehat{S}_{\mathcal{W} \xi} \widehat{\otimes}_{\mathcal{S}} V\right)$. By Lemma 2.2. b it is idempotent and by Theorem 2.5 it is exact. Given any higher extension

$$
V_{*}:=\left(V_{0}=q^{*} M \rightarrow V_{1} \rightarrow \cdots \rightarrow V_{n} \rightarrow V_{n+1}=q^{*} N\right),
$$

$E\left(V_{*}\right)$ is again an $n$-fold extension of $q^{*} N$ by $q^{*} M$ and it comes with a homomorphism $V_{*} \rightarrow E\left(V_{*}\right)$. Hence $V_{*}$ and $E\left(V_{*}\right)$ determine the same equivalence class in $\operatorname{Ext}_{\operatorname{Mod}_{\Omega}(\mathcal{S})}^{n}\left(q^{*} N, q^{*} M\right)$ and $q^{*}: \operatorname{Mod}_{\Omega}\left(\widehat{\mathcal{S}}_{\mathcal{W} \xi}\right) \rightarrow \operatorname{Mod}_{\Omega}(\mathcal{S})$ induces the required isomorphism.

(b) The first isomorphism is a special case of OpSo1, Corollary 3.7.a].

Let $P_{*}$ be a projective resolution of $V$ in $\operatorname{Mod}_{D N \Omega}(\mathcal{S})$. We may assume that $P_{n}=\mathcal{S} \widehat{\otimes} F_{n}$ for some $F_{n} \in D N \Omega$. By Theorem 2.5 $\widehat{S}_{\mathcal{W} \xi} \widehat{\otimes}_{\mathcal{S}} P_{*}=\widehat{S}_{\mathcal{W} \xi} \widehat{\otimes} F_{*}$ is a projective resolution of $\widehat{V}_{\mathcal{W} \xi}$ in $\operatorname{Mod}_{\Omega}\left(\widehat{S}_{\mathcal{W} \xi}\right)$. By Lemma 2.2.d and Lemma 2.4.d

$$
\begin{aligned}
\operatorname{Ext}_{\operatorname{Mod}_{b o r}(\mathcal{S})}^{n}\left(V, q^{*} M\right) & \cong H^{n}\left(\operatorname{Hom}_{\mathcal{S}}\left(\mathcal{S} \widehat{\otimes} F_{*}, q^{*} M\right)\right) \\
& \cong H^{n}\left(\operatorname{Hom}_{\widehat{\mathcal{S}}_{\mathcal{W} \xi}}\left(\widehat{S}_{\mathcal{W} \xi} \widehat{\otimes} F_{*}, M\right)\right) \\
& \cong \operatorname{Ext}_{\operatorname{Mod}_{\Omega}\left(\widehat{\mathcal{S}}_{\mathcal{W} \xi}\right)}^{n}\left(\widehat{V}_{\mathcal{W} \xi}, M\right) .
\end{aligned}
$$

Now let $\widehat{P}_{*}$ be a projective resolution of $\widehat{V}_{\mathcal{W} \xi}$ in $\operatorname{Mod}_{f g}\left(\widehat{\mathcal{S}}_{\mathcal{W} \xi}\right)$. The proof of Lemma 2.4. f shows that

$$
\operatorname{Ext}_{\operatorname{Mod}_{\Omega}\left(\widehat{\mathcal{S}}_{\mathcal{W} \xi}\right)}^{n}\left(\widehat{V}_{\mathcal{W} \xi}, M\right) \cong H^{n}\left(\operatorname{Hom}_{\widehat{\mathcal{S}}_{\mathcal{W} \xi}}\left(\widehat{P}_{*}, M\right)\right) \cong \operatorname{Ext}_{\widehat{\mathcal{S}}_{\mathcal{W} \xi}}^{n}\left(\widehat{V}_{\mathcal{W} \xi}, M\right)
$$

(c) For finite dimensional $V$ it is explained in the proof of OpSo1, Corollary 3.7.b] that it does not matter whether one works with algebraic or with bornological modules to define Ext-groups over $\mathcal{H}$ or $\mathcal{S}$.

\section{Algebras with finite group actions}

This section is of a rather general nature and does not depend on the rest of the paper. We start with a folklore result, which we will use several times. Let $(\pi, V)$ be a finite dimensional complex representation of a finite group $G$. Then $\operatorname{End}_{\mathbb{C}}(V)$ becomes a $G$-representation by defining

$$
g \cdot \phi:=\pi(g) \circ \phi \circ \pi(g)^{-1} \quad \phi \in \operatorname{End}_{\mathbb{C}}(V), g \in G .
$$

Lemma 3.1. Let $\mathbb{C}[G]$ be the right regular $G$-representation, that is, $\rho(g) h=h g^{-1}$. Let $B$ be any complex $G$-algebra and endow $B \otimes \operatorname{End}_{\mathbb{C}}(\mathbb{C}[G])$ with the diagonal $G$ action. Then

$$
\left(B \otimes \operatorname{End}_{\mathbb{C}}(\mathbb{C}[G])\right)^{G} \cong B \rtimes G .
$$


Proof. Denote the action of $g \in G$ on $B$ by $\alpha_{g}$. For a decomposable tensor $f \otimes g \in$ $B \rtimes G$ we define $L(f \otimes g) \in B \otimes \operatorname{End}_{\mathbb{C}}(\mathbb{C}[G])$ by

$$
L(f \otimes g)(h)=\alpha_{h^{-1} g^{-1}}(f) \otimes g h \quad h \in G .
$$

It is easily verified that $L(f \otimes g)$ is $G$-invariant and that $L$ extends to an algebra homomorphism

$$
L: B \rtimes G \rightarrow\left(B \otimes \operatorname{End}_{\mathbb{C}}(\mathbb{C}[G])\right)^{G} .
$$

A straightforward calculation shows that $L$ is invertible with inverse

$$
L^{-1}(b)=\sum_{g \in G} b\left(g^{-1}\right)_{e} \otimes g,
$$

where $b\left(g^{-1}\right)=\sum_{h \in G} b\left(g^{-1}\right)_{h} \otimes h \in B \otimes \mathbb{C}[G]$.

Let $M$ be a smooth manifold, let $T_{m}(M)$ be the tangent space of $M$ at $m$ and $T_{m}^{*}(M)$ the cotangent space. We suppose that the finite group $G$ acts on $M$ by diffeomorphisms. We denote the isotropy group of $m \in M$ by $G_{m}$. For any finite dimensional $G_{m}$-representation $\sigma$ we define the $C^{\infty}(M) \rtimes G$-representation

$$
\operatorname{Ind}_{m} \sigma=\operatorname{Ind}_{C^{\infty}(M) \rtimes G_{m}}^{C^{\infty}(M) \rtimes G} \sigma_{m}
$$

where $\sigma_{m}$ is $\sigma$ regarded as a $C^{\infty}(M) \rtimes G_{m}$-representation with $C^{\infty}(M)$-character $m$.

We let $V$ be a finite dimensional $G$-representation and we endow the algebra $C^{\infty}(M) \otimes \operatorname{End}_{\mathbb{C}}(V)$ with the diagonal $G$-action. Algebras of the form

$$
\left(C^{\infty}(M) \otimes \operatorname{End}_{\mathbb{C}}(V)\right)^{G}
$$

were studied among others in [Sol1] and [Sol2, Section 2.5]. Let $\mathcal{F}_{m}$ be the Fréchet algebra of formal power series at $m \in M$, with complex coefficients. Then $\mathcal{F}_{G m}:=$ $\bigoplus_{m^{\prime} \in G m} \mathcal{F}_{m^{\prime}}$ is naturally a $G$-algebra, so we can again construct

$$
\left(\mathcal{F}_{G m} \otimes \operatorname{End}_{\mathbb{C}}(V)\right)^{G} .
$$

In the proof of Theorem 5.2 we will use some results for such algebras, which we prove here.

Theorem 3.2. Let $V^{\prime}$ be another finite dimensional $G$-representation and assume that, for every irreducible G-representation $W, \operatorname{Hom}_{G}(W, V)=0$ if and only if $\operatorname{Hom}_{G}\left(W, V^{\prime}\right)=0$.

(a) The algebras $\left(C^{\infty}(M) \otimes \operatorname{End}_{\mathbb{C}}(V)\right)^{G}$ and $\left(C^{\infty}(M) \otimes \operatorname{End}_{\mathbb{C}}\left(V^{\prime}\right)\right)^{G}$ are Moritaequivalent, both in the algebraic and in the bornological sense.

(b) Let $\sigma$ be a finite dimensional representation of $G_{m}$. Under the isomorphism from Lemma 3.1 the $C^{\infty}(M) \rtimes G$-representation $\operatorname{Ind}_{m} \sigma$ corresponds to a $\left(C^{\infty}(M) \otimes\right.$ $\left.\operatorname{End}_{\mathbb{C}}(\mathbb{C}[G])\right)^{G}$-representation $\pi(m, \sigma)$ with $C^{\infty}(M)^{G}$-character $G m$, such that

$$
\begin{aligned}
& \operatorname{Hom}_{\left(C^{\infty}(M) \otimes \operatorname{End}_{\mathbb{C}}(\mathbb{C}[G])\right)^{G}\left(\mathbb{C}_{m} \otimes \mathbb{C}[G], \pi(m, \sigma)\right)} \cong \sigma \\
& \cong \operatorname{Hom}_{C^{\infty}(M) \rtimes G}\left(\operatorname{Ind}_{m} \mathbb{C}\left[G_{m}\right], \operatorname{Ind}_{m} \sigma\right) .
\end{aligned}
$$

These are isomorphisms of $G_{m}$-representations with respect to the right regular action of $G_{m}$ on $\mathbb{C}[G]$ and $\mathbb{C}\left[G_{m}\right]$.

(c) Parts (a) and (b) remain valid if we replace $C^{\infty}(M)$ by $\mathcal{F}_{G m}$.

(d) The analogous statements hold if $M$ is a nonsingular complex affine $G$-variety, and we replace $C^{\infty}(M)$ by the ring $\mathcal{O}(M)$ of regular functions on $M$ everywhere. 
Remark. The condition on $V$ and $V^{\prime}$ is called quasi-equivalence in [LePl], where it is related to stable equivalence of certain $C^{*}$-algebras.

Proof. (a) Consider the bimodules

$$
\begin{aligned}
& D_{1}=C^{\infty}(M) \otimes \operatorname{Hom}_{\mathbb{C}}\left(V, V^{\prime}\right), \\
& D_{2}=C^{\infty}(M) \otimes \operatorname{Hom}_{\mathbb{C}}\left(V^{\prime}, V\right) .
\end{aligned}
$$

It is clear that

$$
D_{1} \otimes_{C^{\infty}(M) \otimes \operatorname{End}_{\mathbb{C}}(V)} D_{2} \cong C^{\infty}(M) \otimes \operatorname{End}_{\mathbb{C}}\left(V^{\prime}\right),
$$

and similarly in the reversed order. We will show that (35) remains valid if we take $G$-invariants everywhere. In other words, we claim that the bimodules $D_{1}^{G}$ and $D_{2}^{G}$ implement the desired Morita equivalence. First we check that $D_{1}^{G}$ is projective as a right $\left(C^{\infty}(M) \otimes \operatorname{End}_{\mathbb{C}}(V)\right)^{G}$-module. Choose a $G$-representation $V_{3}$ such that $V^{\prime} \oplus V_{3} \cong V \otimes \mathbb{C}^{d}$ for some $d \in \mathbb{N}$ and write $D_{3}=C^{\infty}(M) \otimes \operatorname{Hom}_{\mathbb{C}}\left(V, V_{3}\right)$. Then

$$
D_{1}^{G} \oplus D_{3}^{G} \cong\left(C^{\infty}(M) \otimes \operatorname{Hom}_{\mathbb{C}}\left(V, V \otimes \mathbb{C}^{d}\right)\right)^{G} \cong\left(C^{\infty}(M) \otimes \operatorname{End}_{\mathbb{C}}(V)\right)^{G} \otimes \mathbb{C}^{d},
$$

so $D_{1}^{G}$ is a finitely generated projective $\left(C^{\infty}(M) \otimes \operatorname{End}_{\mathbb{C}}(V)\right)^{G}$-module, in both the algebraic and the bornological sense. Similarly $D_{2}^{G}$ is a finitely generated projective $\left(C^{\infty}(M) \otimes \operatorname{End}_{\mathbb{C}}\left(V^{\prime}\right)\right)^{G}$-module, so all the below algebraic tensor products are also bornological tensor products.

Multiplication yields a natural map

$$
D_{1}^{G} \otimes\left(C^{\infty}(M) \otimes \operatorname{End}_{\mathbb{C}}(V)\right)^{G} D_{2}^{G} \rightarrow\left(C^{\infty}(M) \otimes \operatorname{End}_{\mathbb{C}}\left(V^{\prime}\right)\right)^{G} .
$$

Take any $m \in M$ and let $I_{G m} \subset C^{\infty}(M)^{G}$ be the maximal ideal $\left\{f \in C^{\infty}(M)^{G}\right.$ | $f(m)=0\}$. Dividing out (36) by $I_{G m}$, we obtain

$$
\operatorname{Hom}_{G_{m}}\left(V, V^{\prime}\right) \otimes_{\operatorname{End}_{G_{m}}\left(V^{\prime}\right)} \operatorname{Hom}_{G_{m}}\left(V^{\prime}, V\right) \rightarrow \operatorname{End}_{G_{m}}(V) .
$$

Decompose the $G_{m}$-representations $V$ and $V^{\prime}$ as

$$
V \cong \bigoplus_{\rho \in \operatorname{Irr}\left(G_{m}\right)} \rho \otimes \mathbb{C}^{m_{\rho}}, \quad V^{\prime} \cong \bigoplus_{\rho \in \operatorname{Irr}\left(G_{m}\right)} \rho \otimes \mathbb{C}^{m_{\rho}^{\prime}}
$$

The assumption of the theorem implies that $m_{\rho}=0$ if and only if $m_{\rho}^{\prime}=0$. As

$$
\operatorname{End}_{G_{m}}\left(V^{\prime}\right) \cong \bigoplus_{\rho \in \operatorname{Irr}\left(G_{m}\right)} \operatorname{End}_{G_{m}}(\rho) \otimes \operatorname{End}_{\mathbb{C}}\left(\mathbb{C}^{m_{\rho}^{\prime}}\right)
$$

the left hand side of (37) is isomorphic to

$$
\begin{array}{r}
\bigoplus_{\rho \in \operatorname{Irr}\left(G_{m}\right)} \operatorname{End}_{G_{m}}(\rho) \otimes \operatorname{Hom}_{\mathbb{C}}\left(\mathbb{C}^{m_{\rho}}, \mathbb{C}^{m_{\rho}^{\prime}}\right) \otimes_{\operatorname{End}_{G_{m}}\left(V^{\prime}\right)} \bigoplus_{\rho \in \operatorname{Irr}\left(G_{m}\right)} \operatorname{End}_{G_{m}}(\rho) \otimes \operatorname{Hom}_{\mathbb{C}}\left(\mathbb{C}^{m_{\rho}^{\prime}}, \mathbb{C}^{m_{\rho}}\right) \\
\cong \bigoplus_{\rho \in \operatorname{Irr}(G)} \operatorname{End}_{G_{m}}(\rho) \otimes \operatorname{End}_{\mathbb{C}}\left(\mathbb{C}^{m_{\rho}}\right) \cong \operatorname{End}_{G_{m}}(V) .
\end{array}
$$

Hence (37) is a bijection for all $m \in M$, which implies that (36) is injective. The image of (36) is a two-sided ideal of $\left(C^{\infty}(M) \otimes \operatorname{End}_{\mathbb{C}}\left(V^{\prime}\right)\right)^{G}$, which is dense in the sense that for every $m \in M$ the algebra and its ideal have the same set of values at $m$. Consequently (36) is bijective, and an isomorphism of $\left(C^{\infty}(M) \otimes \operatorname{End}_{\mathbb{C}}\left(V^{\prime}\right)\right)^{G}$ bimodules. 
(b) Let $J_{G m}$ and $J_{G m}^{\prime}$ be the ideals of $C^{\infty}(M) \rtimes G$ and $\left(C^{\infty}(M) \otimes \operatorname{End}_{\mathbb{C}}(\mathbb{C}[G])\right)^{G}$ generated by $I_{G m}$. As the isomorphism from Lemma 3.1 preserves the central characters, the representations under consideration factor through the quotients

$$
\begin{gathered}
\left(C^{\infty}(M) \rtimes G\right) / J_{G m} \cong \mathbb{C}[G m] \rtimes G \cong \operatorname{End}_{\mathbb{C}}(\mathbb{C}[G m]) \otimes \mathbb{C}\left[G_{m}\right], \\
\left.\left(C^{\infty}(M) \otimes \operatorname{End}_{\mathbb{C}}(\mathbb{C}[G])\right)\right)^{G} / J_{G m}^{\prime} \cong\left(\bigoplus_{g \in G / G_{m}} \operatorname{End}_{\mathbb{C}}(\mathbb{C}[G])\right)^{G} \cong \operatorname{End}_{G_{m}}(\mathbb{C}[G]) .
\end{gathered}
$$

The two rightmost algebras are isomorphic via a bijection $G \rightarrow G m \times G_{m}$ which is equivariant with respect to the right $G_{m}$-action. The representations corresponding to (38) are

$$
\begin{array}{ccc}
\operatorname{Ind}_{m} \sigma & \mathbb{C}[G m] \otimes \sigma & \mathbb{C}[G m] \otimes \sigma, \\
\pi(m, \sigma) & \mathbb{C}[G] \otimes_{\mathbb{C}\left[G_{m}\right]} \sigma & \mathbb{C}[G] \otimes_{\mathbb{C}\left[G_{m}\right]} \sigma .
\end{array}
$$

In the special case where $\sigma$ is the (right) regular representation of $G_{m}$, we see that $\pi\left(n, \mathbb{C}\left[G_{m}\right]\right) \cong \mathbb{C}_{m} \otimes \mathbb{C}[G]$. Therefore

$$
\begin{aligned}
& \operatorname{Hom}_{C \infty}(M) \rtimes G\left(\operatorname{Ind}_{m} \mathbb{C}\left[G_{m}\right], \operatorname{Ind}_{m} \sigma\right) \cong \\
& \operatorname{Hom}_{\left(C^{\infty}(M) \otimes \operatorname{End}_{\mathbb{C}}(\mathbb{C}[G])\right)}{ }^{G}\left(\mathbb{C}_{m} \otimes \mathbb{C}[G], \pi(m, \sigma)\right) \cong \\
& \operatorname{Hom}_{\operatorname{End}_{G_{m}}(\mathbb{C}[G])}\left(\mathbb{C}[G], \mathbb{C}[G] \otimes_{\mathbb{C}\left[G_{m}\right]} \sigma\right) \cong \operatorname{Hom}_{\mathbb{C}\left[G_{m}\right]}\left(\mathbb{C}\left[G_{m}\right], \sigma\right) .
\end{aligned}
$$

Notice that all these steps preserve the right action of $G_{m}$ on $\mathbb{C}[G]$ or $\mathbb{C}\left[G_{m}\right]$. The last term is $G_{m}$-equivariantly isomorphic to $\sigma$ via the evaluation map $\phi \mapsto \phi(1)$.

(c) Let $d \in \mathbb{N}$ and divide (36) out by $I_{G m}^{d}$. It remains bijective, which implies that

$$
\begin{array}{r}
\left(\mathcal{F}_{G m} / I_{G m}^{d} \otimes \operatorname{Hom}_{\mathbb{C}}\left(V, V^{\prime}\right)\right)^{G_{m}} \otimes_{\left(\mathcal{F}_{G m} \otimes \operatorname{End}_{\mathbb{C}}(V)\right)^{G}}\left(\mathcal{F}_{G m} / I_{G m}^{d} \otimes \operatorname{Hom}_{\mathbb{C}}\left(V, V^{\prime}\right)\right)^{G_{m}} \cong \\
\left(\mathcal{F}_{G m} / I_{G m}^{d} \otimes \operatorname{End}_{\mathbb{C}}\left(V^{\prime}\right)\right)^{G_{m}} .
\end{array}
$$

Hence the bimodules $\left(\mathcal{F}_{G m} \otimes \operatorname{Hom}_{\mathbb{C}}\left(V, V^{\prime}\right)\right)^{G}$ and $\left(\mathcal{F}_{G m} \otimes \operatorname{Hom}_{\mathbb{C}}\left(V, V^{\prime}\right)\right)^{G}$ provide the required Morita equivalence. Knowing this, it is obvious that the proof of part (b) also works with formal power series instead of smooth functions.

(d) All the previous arguments go through without change in the affine algebraic case.

3.1. Ext-groups and the Yoneda product. Let $M$ be a nonsingular complex affine variety. It is well known that (commutative) regular local rings admit Koszulresolutions and that these can be used to determine Ext-spaces of suitable modules. This leads for example to

$$
\operatorname{Ext}_{\mathcal{O}(M)}^{*}\left(\mathbb{C}_{m}, \mathbb{C}_{m}\right) \cong \bigwedge^{*} T_{m}(M),
$$

and with a little more work one can show that the Yoneda product on the left hand side corresponds to the $\wedge$-product on the right hand side. It turns out that this can be generalized to crossed products with finite groups. We formulate the next result with complex affine $G$-varieties, but using [Was, Proposition 6] and Subsection 2.1 (for completions in the context of Fréchet algebras) it is allowed to replace $\mathcal{O}(M)$ by smooth functions on a manifold.

Theorem 3.3. Let $V, V^{\prime}$ be finite dimensional $G_{m}$-representations. 
(a) $\operatorname{Ext}_{\mathcal{O}(M) \rtimes G}^{n}\left(\operatorname{Ind}_{m} V, \operatorname{Ind}_{m} V^{\prime}\right)$ is isomorphic to

$$
\operatorname{Hom}_{G_{m}}\left(V \otimes \bigwedge^{n} T_{m}^{*}(M), V^{\prime}\right) \cong\left(\operatorname{Hom}_{\mathbb{C}}\left(V, V^{\prime}\right) \otimes \bigwedge^{n} T_{m}(M)\right)^{G_{m}}
$$

(b) The Yoneda product on $\operatorname{Ext}_{\mathcal{O}(M) \rtimes G}^{*}\left(\operatorname{Ind}_{m} V, \operatorname{Ind}_{m} V\right)$ agrees with the usual product on $\left(\operatorname{End}_{\mathbb{C}}(V) \otimes \bigwedge^{*} T_{m}(M)\right)^{G_{m}}$.

(c) For the regular representation $V=\mathbb{C}\left[G_{m}\right]$ we have $\operatorname{Ind}_{m} V=\operatorname{Ind}_{\mathcal{O}(M)}^{\mathcal{O}(M) \rtimes G} \mathbb{C}_{m}$ and

$$
\operatorname{Ext}_{\mathcal{O}(M) \rtimes G}^{*}\left(\operatorname{Ind}_{\mathcal{O}(M) \rtimes}^{\mathcal{O}(M)} \mathbb{C}_{m}, \operatorname{Ind}_{\mathcal{O}(M)}^{\mathcal{O}(M) \rtimes G} \mathbb{C}_{m}\right) \cong \bigwedge^{*} T_{m}(M) \rtimes G_{m}
$$

as graded algebras.

(d) Let $\mathcal{F}_{G m}$ be the completion of $\mathcal{O}(M)$ with respect to the powers of the ideal $I_{G m}:=\{f \in \mathcal{O}(M): f(g m)=0 \forall g \in G\}$. Endow it with the Fréchet topology of a complex power series ring. Then (a), (b) and (c) remain valid if we replace $\operatorname{Mod}(\mathcal{O}(M) \rtimes G)$ by any of the following categories: $\operatorname{Mod}\left(\mathcal{F}_{G m} \rtimes\right.$ $G), \operatorname{Mod}_{F r e ́}\left(\mathcal{F}_{G m} \rtimes G\right), \operatorname{Mod}_{\text {bor }}\left(\mathcal{F}_{G m} \rtimes G\right)$.

Proof. (a) First we copy a part of the proof of [OpSo1, Theorem 3.2]. Let $V_{m}$ be $V$ considered as a $C^{\infty}(M) \rtimes G_{m}$ module with $C^{\infty}(M)$-character $m$. By Frobenius reciprocity and because two $\mathcal{O}(M) \rtimes G_{m}$-representations with different central characters admit only trivial extensions

$$
\begin{aligned}
\operatorname{Ext}_{\mathcal{O}(M) \rtimes G}^{n}\left(\operatorname{Ind}_{m} V, \operatorname{Ind}_{m} V^{\prime}\right) & \cong \operatorname{Ext}_{\mathcal{O}(M) \rtimes G_{m}}^{n}\left(V_{m}, \operatorname{Ind}_{m} V^{\prime}\right) \\
& \cong \operatorname{Ext}_{\mathcal{O}(M) \rtimes G_{m}}^{n}\left(V_{m}, V_{m}^{\prime}\right) .
\end{aligned}
$$

Let $\mathcal{F}_{m}$ be the completion of $\mathcal{O}(M)$ with respect to the powers of the ideal

$$
I_{m}:=\{f \in \mathcal{O}(M): f(m)=0\} .
$$

It annihilates $V_{m}$, so

$$
\left(\mathcal{F}_{m} \rtimes G_{m}\right) \otimes_{\mathcal{O}(M) \rtimes G_{m}} V_{m}=V_{m}
$$

as $\mathcal{O}(M) \rtimes G_{m}$-modules. Since $\mathcal{F}_{m}$ is flat over $\mathcal{O}(M)$ [Eis, Theorem 7.2.b], so is $\mathcal{F}_{m} \rtimes G$ over $\mathcal{O}(M) \rtimes G$. Therefore the functor $\mathcal{F}_{m} \rtimes G \otimes_{\mathcal{O}(M) \rtimes G}$ ? induces an isomorphism

$$
\operatorname{Ext}_{\mathcal{O}(M) \rtimes G_{m}}^{n}\left(V_{m}, V_{m}^{\prime}\right) \cong \operatorname{Ext}_{\mathcal{F}_{m} \rtimes G_{m}}^{n}\left(V_{m}, V_{m}^{\prime}\right) .
$$

Because the $G_{m}$-module $I_{m}^{2}$ has finite codimension in $\mathcal{O}(M)$, there exists a $G_{m^{-}}$ submodule $E_{m} \subset \mathcal{O}(M)$ such that

$$
\mathcal{O}(M)=\mathbb{C} \oplus E_{m} \oplus I_{m}^{2}
$$

As a $G_{m}$-module $E_{m}$ is the cotangent space to $M$ at $m$, more or less by definition of the latter. Since $\mathcal{F}_{m}$ is a local ring we have $\mathcal{F}_{m} E_{m}=\mathcal{F}_{m} I_{m}$, by Nakayama's Lemma. Any finite dimensional $G_{m}$-module is projective, so

$$
V \otimes \bigwedge^{n} E_{m} \otimes \mathcal{F}_{m}=\operatorname{Ind}_{G_{m}}^{\mathcal{F}_{m} \rtimes G_{m}}\left(V \otimes \bigwedge^{n} E_{m}\right)
$$

is a projective $\mathcal{F}_{m} \rtimes G_{m}$-module, for all $n \in \mathbb{N}$. With these modules we construct a resolution of $V_{m}$. Define $\mathcal{F}_{m} \rtimes G_{m}$-module maps

$$
\begin{array}{ll}
\delta_{n}: V \otimes \bigwedge^{n} E_{m} \otimes \mathcal{F}_{m} & \rightarrow V \otimes \bigwedge^{n-1} E_{m} \otimes \mathcal{F}_{m}, \\
\delta_{n}\left(v \otimes e_{1} \wedge \cdots \wedge e_{n} \otimes f\right) & =\sum_{i=1}^{n}(-1)^{i-1} v \otimes e_{1} \wedge \cdots \wedge e_{i-1} \wedge e_{i+1} \wedge \cdots \wedge e_{n} \otimes e_{i} f \\
\delta_{0}: V \otimes \mathcal{F}_{m} & \rightarrow V_{m}, \\
\delta_{0}(v \otimes f) & =f(m) v .
\end{array}
$$


This makes

$$
\left(V \otimes \bigwedge^{*} E_{m} \otimes \mathcal{F}_{m}, \delta_{*}\right)
$$

into an augmented differential complex. Notice that in $\operatorname{Mod}\left(\mathcal{F}_{m}\right)$ this just the Koszul resolution of

$$
V \otimes \mathcal{F}_{m} / I_{m} \mathcal{F}_{m}=V_{m}
$$

Therefore (42) is the required projective resolution of $V_{m}$. Since $V_{m}^{\prime}$ admits the $\mathcal{F}_{m^{-}}$ character $m$ it is annihilated by $E_{m}$, and in particular $\phi \circ \delta_{n+1}=0$ for every $\mathcal{F}_{m} \rtimes G_{m^{-}}$ module homomorphism $\phi: V \otimes \bigwedge^{n} E_{m} \otimes \mathcal{F}_{m} \rightarrow V_{m}^{\prime}$. (The authors overlooked this important point while writing OpSo1.) Consequently all the differentials in the complex

vanish and

$$
\left(\operatorname{Hom}_{\mathcal{F}_{m} \ltimes G_{m}}\left(V \otimes \bigwedge^{*} E_{m} \otimes \mathcal{F}_{m}, V_{m}^{\prime}\right), \operatorname{Hom}\left(\delta_{*}, \operatorname{id}_{V_{m}^{\prime}}\right)\right)
$$

$$
\begin{aligned}
\operatorname{Ext}_{\mathcal{O}(M) \rtimes G_{m}}^{n}\left(V_{m}, V_{m}^{\prime}\right) & =\operatorname{Hom}_{\mathcal{F}_{m} \ltimes G_{m}}\left(V \otimes \bigwedge^{n} E_{m} \otimes \mathcal{F}_{m}, V_{m}^{\prime}\right) \\
& \cong \operatorname{Hom}_{G_{m}}\left(V \otimes \bigwedge^{n} E_{m}, V^{\prime}\right) .
\end{aligned}
$$

Recall that $E_{m}$ can be identified with $T_{m}^{*}(M)$, so the dual space of $\wedge^{n} E_{m}$ is $\bigwedge^{n} T_{m}(M)$. Thus we can express the right hand side of (43) as

$$
\operatorname{Hom}_{G_{m}}\left(V, \bigwedge^{n} T_{m}(M) \otimes V^{\prime}\right) \cong\left(\operatorname{Hom}_{\mathbb{C}}\left(V, V^{\prime}\right) \otimes \bigwedge^{n} T_{m}(M)\right)^{G_{m}} .
$$

(b) We recall from [Mac, Section III.6] how the Yoneda product can be defined using resolutions of modules. Let $Y$ be a module over any unital ring $R$ and let $P_{*} \rightarrow Y$ be a projective resolution. By definition $\operatorname{Ext}_{R}^{n}(Y, Y)=H^{n}\left(\operatorname{Hom}_{R}\left(P_{*}, Y\right)\right)$. Given any $\phi \in \operatorname{Hom}_{R}\left(P_{n}, Y\right)$ with $\phi \circ \delta_{n+1}=0$, the projectivity of $P_{n}$ guarantees that we can extend it to a chain map $\phi_{*} \in \operatorname{Hom}_{R}\left(P_{*}, P_{*}\right)$ of degree $-n$ :

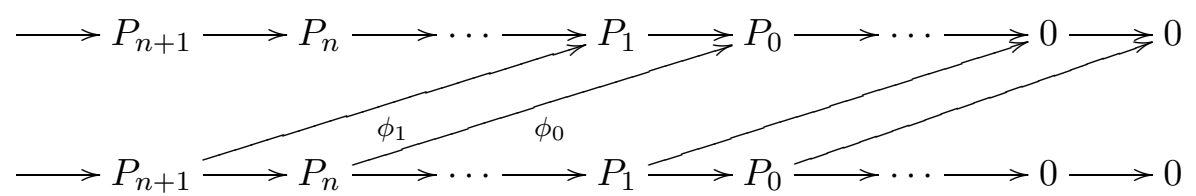

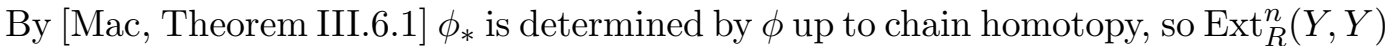
can be regarded as $\operatorname{End}\left(P_{*}, P_{*}\right)$ modulo homotopy. Now $\operatorname{End}\left(P_{*}, P_{*}\right)$ is a graded algebra, and by [Mac, Theorem III.6.4 and exercise III.6.2] its multiplication corresponds to the Yoneda product on $\operatorname{Ext}_{R}^{*}(Y, Y)$.

In our setting, by part (a) every

$$
\phi \in \operatorname{Ext}_{\mathcal{O}(M) \rtimes G}^{n}\left(\operatorname{Ind}_{m} V, \operatorname{Ind}_{m} V\right) \cong \operatorname{Ext}_{\mathcal{F}_{m} \rtimes G_{m}}^{n}\left(V_{m}, V_{m}\right)
$$

can be taken of the form

$$
\phi=\sum_{j} \psi_{j} \otimes \lambda_{j} \in\left(\operatorname{End}_{\mathbb{C}}(V) \otimes \bigwedge^{n} T_{m}(M)\right)^{G_{m}} .
$$

Above we showed that (42) is a projective resolution of $V_{m}$, so we want to lift $\phi$ to $\phi_{*} \in \operatorname{End}_{\mathcal{F}_{M} \rtimes G_{m}}\left(V \otimes \bigwedge^{*} E_{m} \otimes \mathcal{F}_{m}\right)$. We claim that this can be done by defining

$$
\phi_{m+n}(v \otimes \omega \otimes f)=\sum_{j} \psi_{j}(v) \otimes \imath\left(\lambda_{j}\right) \omega \otimes f,
$$

where $\imath\left(\lambda_{j}\right) \omega$ means that $\lambda_{j}$ is inserted in the last $n$ entries of $\omega \in \bigwedge^{n+m} E_{m} \cong$ $\bigwedge^{n+m} T_{m}^{*}(M)$. It is clear that $\phi_{*}$ is $\mathcal{F}_{m} \rtimes G_{m}$-linear whenever $\phi$ is so. To check that $\phi_{*}$ is a chain map, we may just as well work inside $\operatorname{End}_{\mathcal{F}_{m}}\left(V \otimes \bigwedge^{*} E_{m} \otimes \mathcal{F}_{m}\right)$. Then we can reduce the verification to $\phi$ of the form $\psi \otimes t_{1} \wedge \cdots \wedge t_{n}$ with $\psi \in$ 
$\operatorname{End}_{\mathbb{C}}(V)$ and $t_{i} \in T_{m}(M)$ linearly independent. Furthermore it suffices to consider $\omega=e_{1} \wedge \cdots \wedge e_{n+m}$ for $e_{k} \in E_{m}$ such that $\left\langle e_{k}, t_{i}\right\rangle=0$ for all $k \leq m$ and all $i$. Now we calculate

$$
\begin{aligned}
\delta_{m} \phi_{m} & (v \otimes \omega \otimes f)=\delta_{m}\left(\psi(v) \otimes e_{1} \wedge \cdots \wedge e_{m} \operatorname{det}\left(\left\langle e_{k+m}, t_{i}\right\rangle\right)_{i, k=1}^{n} \otimes f\right) \\
& =\psi(v) \otimes \sum_{i=1}^{m}(-1)^{i} e_{1} \wedge \cdots \wedge e_{i-1} \wedge e_{i+1} \wedge \cdots \wedge e_{n} \operatorname{det}\left(\left\langle e_{k+m}, t_{i}\right\rangle\right)_{i, k=1}^{n} \otimes e_{i} f \\
& =\psi(v) \otimes \sum_{i=1}^{m+n}(-1)^{i-1} \imath\left(t_{1} \wedge \cdots \wedge t_{n}\right) e_{1} \wedge \cdots \wedge e_{i-1} \wedge e_{i+1} \wedge \cdots \wedge e_{n+m} \otimes e_{i} f \\
& =\phi_{m-1}\left(\sum_{i=1}^{n+m}(-1)^{i-1} v \otimes e_{1} \wedge \cdots \wedge e_{i-1} \wedge e_{i+1} \wedge \cdots \wedge e_{n+m} \otimes e_{i} f\right) \\
& =\phi_{m-1} \delta_{m+n}(v \otimes \omega \otimes f) .
\end{aligned}
$$

With these explicit lifts available, we can determine the Yoneda product. Since

$$
\left(\psi_{j} \otimes \imath\left(\lambda_{j}\right) \otimes \operatorname{id}_{\mathcal{F}_{m}}\right) \circ\left(\psi_{k}^{\prime} \otimes \imath\left(\lambda_{k}^{\prime}\right) \otimes \operatorname{id}_{\mathcal{F}_{m}}\right)=\psi_{j} \psi_{k}^{\prime} \otimes \imath\left(\lambda_{j} \wedge \lambda_{k}^{\prime}\right) \otimes \operatorname{id}_{\mathcal{F}_{m}}
$$

we obtain

$$
\phi \circ \phi^{\prime}=\left(\sum_{j} \psi_{j} \otimes \lambda_{j}\right) \circ\left(\sum_{k} \psi_{k}^{\prime} \otimes \lambda_{k}^{\prime}\right)=\sum_{j, k} \psi_{j} \psi_{k}^{\prime} \otimes \lambda_{j} \wedge \lambda_{k}^{\prime} .
$$

(c) This follows from (b) and Lemma 3.1

(d) By the Chinese remainder theorem $\mathcal{F}_{G m} \cong \bigoplus_{m^{\prime} \in G m} \mathcal{F}_{m^{\prime}}$, so (39) becomes

$$
\operatorname{Ext}_{\mathcal{F}_{G m} \rtimes G}^{n}\left(\operatorname{Ind}_{m} V, \operatorname{Ind}_{m} V^{\prime}\right) \cong \operatorname{Ext}_{\mathcal{F}_{m} \rtimes G_{m}}^{n}\left(V_{m}, V_{m}^{\prime}\right) .
$$

Thus the proof of (a), (b) and (c) also applies to $\mathcal{F}_{G m} \rtimes G$. The remaining subtlety is that in $\operatorname{Mod}_{b o r}\left(\mathcal{F}_{m} \rtimes G_{m}\right)$ exact sequences are admissible if and only if they admit a bounded linear splitting. Hence the free modules in this category are of the form $\mathcal{F}_{m} \rtimes G_{m} \widehat{\otimes}_{\mathbb{C}} F$, where $F$ is any bornological vector space. Since $V \otimes \bigwedge^{*} E_{m}$ is a finite dimensional $G_{m}$-representation, it is a direct summand of $\mathbb{C}\left[G_{m}\right]^{d}$ for some $d \in \mathbb{N}$. Hence all the modules $V \otimes \bigwedge^{n} E_{m} \otimes \mathcal{F}_{m}$ of the Koszul resolution of $V_{m}$ are direct summands of $\mathbb{C}\left[G_{m}\right]^{d} \otimes \mathcal{F}_{m}=\left(\mathcal{F} \rtimes G_{m}\right) \widehat{\otimes}_{\mathbb{C}} \mathbb{C}^{d}$, which means that they are projective in both the algebraic and the bornological sense.

Futhermore we have to check that the projective resolution (42) admits a bounded linear splitting. Since we dealing with Fréchet spaces, bounded is the same as continuous. Via (41) we can identify $\mathcal{F}_{m}$ with the formal completion of the symmetric algebra of $E_{m}$. This enables us to define the De Rham differential $D: \mathcal{F}_{m} \rightarrow$ $\bigwedge^{1} E_{m} \otimes \mathcal{F}_{m}$, which is easily seen to be continuous with respect to the Fréchet topology. We define $D_{n}: V \otimes \bigwedge^{n} E_{m} \otimes \mathcal{F}_{m} \rightarrow V \otimes \bigwedge^{n+1} E_{m} \otimes \mathcal{F}_{m}$ by

$$
D_{n}\left(v \otimes e_{1} \wedge \cdots \wedge e_{n} \otimes f\right)=(-1)^{n} v \otimes e_{1} \wedge \cdots \wedge e_{n} \wedge D f
$$

and on the augmentation $V_{m}$ we take $D_{-1}(v)=v \otimes 1$. With a straightforward calculation one checks that

$$
\left(D_{n-1} \delta_{n}+\delta_{n+1} D_{n}\right)\left(\left(v \otimes e_{1} \wedge \cdots \wedge e_{n} \otimes f\right)=(n+g) v \otimes e_{1} \wedge \cdots \wedge e_{n} \otimes f\right.
$$

for $f \in \mathcal{F}_{m}$ homogeneous of degree $g$, except for $n=g=0$, in which case

$$
\left(D_{-1} \delta_{0}+\delta_{1} D_{0}\right)(v \otimes 1)=v \otimes 1 .
$$


Since our resolution is the direct product of differential complexes indexed by the degree $n+g$, this shows that $D_{*} \delta_{*}+\delta_{*} D_{*}$ is invertible. Hence $D$ is the desired continuous contraction.

\section{Analytic R-Groups}

We recall the definition of the analytic R-group from DeOp2. Let $\delta$ be a discrete series representation of $\mathcal{H}_{P}$ with central character $W\left(R_{P}\right) r_{\delta} \in T_{P} / W\left(R_{P}\right)$. Recall that a parabolic subsystem of $R_{0}$ is a subset $R_{Q} \subset R_{0}$ satisfying $R_{Q}=R_{0} \cap \mathbb{R} R_{Q}$. We let $Q$ denote the basis of $R_{Q}$ inside the positive subset $R_{Q,+}:=R_{Q} \cap R_{0,+}$. We call $R_{Q}$ standard if $Q \subset F_{0}$, in which case these notions agree with (9). The set of roots $R_{0} \backslash R_{P}$ is a disjoint union of subsets of the form $R_{Q} \backslash R_{P}$ where $R_{Q} \subset R_{0}$ runs over the collection $\mathcal{P}_{\text {min }}^{P}$ of minimal parabolic subsystems properly containing $R_{P}$. We define $\alpha_{Q} \in R_{0,+}$ by $Q=\left\{P, \alpha_{Q}\right\}$ and $\alpha_{Q}^{P}=\left.\alpha_{Q}\right|_{\mathfrak{a}^{P}} \in \mathfrak{a}^{P, *}$ for $R_{Q} \in \mathcal{P}_{\text {min }}^{P}$. By the integrality properties of the root system $R_{0}$ we see that

$$
\left\{\left.\alpha\right|_{\mathfrak{a}^{P}}: \alpha \in R_{Q}\right\} \subset \mathbb{Z} \alpha_{Q}^{P} .
$$

Clearly $\alpha_{Q}^{P}$ is a character of $T^{P}$ whose kernel contains the codimension one subtorus $T^{Q} \subset T^{P}$. For each $R_{Q} \in \mathcal{P}_{\text {min }}^{P}$ we define a $W\left(R_{P}\right)$-invariant rational function on $T$ by

$$
c_{Q}^{P}(t):=\prod_{\alpha \in R_{Q,+} \backslash R_{P,+}} c_{\alpha}(t),
$$

where $c_{\alpha}: T \rightarrow \mathbb{C}$ denotes the rank one $c$-function associated to $\alpha \in R_{0}$ and the parameter function $q$ of $\mathcal{H}$, see e.g. DeOp1, Appendix 9]. This $c_{\alpha}$ plays an important role in the representation theory of $\mathcal{H}$, as it governs the behaviour of the intertwining operator $I_{s_{\alpha}}$ associated to the reflection $s_{\alpha}$. Roughly speaking, singularities of $I_{s_{\alpha}}$ correspond to the zeros of $c_{\alpha}$, while $I_{s_{\alpha}}$ tends to be a scalar operator on principal series representations parametrized by poles of $c_{\alpha}$.

For any $\alpha \in R_{Q} \backslash R_{P}$ the function $c_{\alpha}$ is a nonconstant rational function on any coset of the form $r T^{P}$. In particular $c_{Q}^{P}$ is regular on a nonempty Zariski-open subset of such a coset. By the $W\left(R_{P}\right)$-invariance we see that for $t \in T^{P}$ and $r \in W\left(R_{P}\right) r_{\delta}$, the value of this function at $r t$ is independent of the choice of $r \in W\left(R_{P}\right) r_{\delta}$. The resulting rational function $t \mapsto c_{Q}^{P}(r t)$ on $T^{P}$ is clearly constant along the cosets of the codimension one subtorus $T^{Q} \subset T^{P}$. We define the set of mirrors $\mathcal{M}_{Q}^{P, \delta}$ associated to $R_{Q} \in \mathcal{P}_{\min }^{P}$ to be the set of connected components of the intersection of the set of poles of this rational function with the unitary part $T_{u n}^{P}$ of $T^{P}$. We put

$$
\mathcal{M}^{P, \delta}=\bigsqcup_{R_{Q} \in \mathcal{P}_{\min }^{P}} \mathcal{M}_{Q}^{P, \delta}
$$

for the set of all mirrors in $T_{u n}^{P}$ associated to the pair $(P, \delta)$. Given $M \in \mathcal{M}^{P, \delta}$ we denote by $R_{Q^{M}} \subset R_{0}$ the unique element of $\mathcal{P}_{\text {min }}^{P}$ such that $M \in \mathcal{M}_{Q^{M}}^{P, \delta}$. Thus any mirror $M \in \mathcal{M}^{P, \delta}$ is a connected component of a hypersurface of $T_{u n}^{P}$ of the form $\alpha_{Q^{M}}^{P}=$ constant. Observe that for a fixed pair $(P, \delta)$ the set $\mathcal{M}^{P, \delta}$ is finite (and possibly empty).

For every mirror $M \in \mathcal{M}^{P, \delta}$ there exists, by DeOp2, Theorem 4.3.i], a unique $s_{M} \in \mathcal{W}_{P, \delta}$ (i.e. $s_{M} \in \mathcal{W}_{P, P}$ and $\delta \simeq \delta \circ \psi_{s_{M}}^{-1}$ ) which acts on $T_{u n}^{P}$ as the reflection in 
$M$. For $\xi=(P, \delta, t) \in \Xi_{\text {un }}$ let $\mathcal{M}_{\xi}$ be the collection of mirrors $M \in \mathcal{M}^{P, \delta}$ containing $t$. We define

$$
R_{\xi}=\left\{ \pm \alpha_{Q^{M}}^{P}: M \in \mathcal{M}_{\xi}\right\} \quad \text { and } \quad R_{\xi}^{+}=\left\{\alpha_{Q^{M}}^{P}: M \in \mathcal{M}_{\xi}\right\} .
$$

Then it follows from DeOp2, Proposition 4.5] and (44) that $R_{\xi}$ is a root system in $\mathfrak{a}^{P, *}$ and that $R_{\xi}^{+} \subset R_{\xi}$ is a positive subset. Its Weyl group $W\left(R_{\xi}\right)$ is generated by the reflections $s_{M}$ with $M \in \mathcal{M}_{\xi}$, so it can be realized as a subgroup of $\mathcal{W}_{\xi}=\{w \in$ $\mathcal{W}: w(\xi)=\xi\}$. The R-group of $\xi \in \Xi_{\text {un }}$ is defined as

$$
\mathfrak{R}_{\xi}=\left\{w \in \mathcal{W}_{\xi}: w\left(R_{\xi}^{+}\right)=R_{\xi}^{+}\right\},
$$

and by [DeOp2, Proposition 4.7] it is a complement to $W\left(R_{\xi}\right)$ in $\mathcal{W}_{\xi}$ :

$$
\mathcal{W}_{\xi}=\mathfrak{R}_{\xi} \ltimes W\left(R_{\xi}\right) .
$$

With these notions one can state the analogue of the Knapp-Stein linear independence theorem [KnSt, Theorem 13.4] for affine Hecke algebras. For reductive $p$-adic groups the result is proven in [Sil], see also [Art, Section 2].

Theorem 4.1. Let $\xi \in \Xi_{\text {un }}$.

(a) For $w \in \mathcal{W}_{\xi}$ the intertwiner $\pi(w, \xi)$ is scalar if and only if $w \in W\left(R_{\xi}\right)$.

(b) There exists a 2-cocycle $\kappa_{\xi}$ (depending on the normalization of the intertwining operators $\pi(w, \xi)$ for $\left.w \in \mathcal{W}_{\xi}\right)$ of $\mathfrak{R}_{\xi}$ such that $\operatorname{End}_{\mathcal{H}}(\pi(\xi))$ is isomorphic to the twisted group algebra $\mathbb{C}\left[\mathfrak{R}_{\xi}, \kappa_{\xi}\right]$.

(c) Given the normalization of the intertwining operators, there is a unique bijection

$$
\operatorname{Irr}\left(\mathbb{C}\left[\Re_{\xi}, \kappa_{\xi}\right]\right) \rightarrow \operatorname{Irr}_{\mathcal{W} \xi}(\mathcal{S}): \rho \mapsto \pi(\xi, \rho),
$$

such that $\pi(\xi) \cong \bigoplus_{\rho} \pi(\xi, \rho) \otimes \rho$ as $\mathcal{H} \otimes \mathbb{C}\left[\Re_{\xi}, \kappa_{\xi}\right]$-modules.

(d) Let $\operatorname{Mod}_{f, \text { un }, \mathcal{W} \xi}(\mathcal{S})$ be the category of finite dimensional unitary $\mathcal{S}$-representations that admit the central character $\mathcal{W} \xi$. The contravariant functor

$$
\begin{array}{cl}
\Phi_{\xi}^{*}: \operatorname{Mod}_{f, u n, \mathcal{W} \xi}(\mathcal{S}) & \rightarrow \operatorname{Mod}_{f}\left(\mathbb{C}\left[\Re_{\xi}, \kappa_{\xi}\right]\right), \\
\pi & \mapsto \operatorname{Hom}_{\mathcal{H}}(\pi, \pi(\xi))
\end{array}
$$

is a duality of categories, with quasi-inverse $\rho \mapsto \pi(\xi, \rho):=\operatorname{Hom}_{\mathbb{C}\left[\Re_{\xi}, \kappa_{\xi}\right]}(\rho, \pi(\xi))$.

Proof. Part (a) is DeOp2, Theorem 5.4], parts (b) and (c) are DeOp2, Theorem 5.5] and part (d) is Opd2, Theorem 5.13].

Recall that $\mathbb{C}\left[\Re_{\xi}, \kappa_{\xi}\right]$ has a canonical basis $\left\{T_{r} \mid r \in \mathfrak{R}_{\xi}\right\}$ and that its multiplication is given by

$$
T_{r} T_{r^{\prime}}=\kappa_{\xi}\left(r, r^{\prime}\right) T_{r r^{\prime}} \quad r, r^{\prime} \in \mathfrak{R}_{\xi}
$$

Via a trace map $\Phi_{\xi}^{*}(\pi)$ is naturally identified with the dual space of

$$
\Phi_{\xi}(\pi):=\operatorname{Hom}_{\mathcal{H}}(\pi(\xi), \pi)=\pi(\xi)^{*} \otimes_{\mathcal{H}} \pi .
$$

Given that $\Phi_{\xi}^{*}(\pi)$ is a $\mathbb{C}\left[\Re_{\xi}, \kappa_{\xi}\right]$-representation, (50) shows that $\Phi_{\xi}(\pi)$ is a representation of $\mathbb{C}\left[\Re_{\xi}, \kappa_{\xi}^{-1}\right]$. Thus Theorem 4.1,d is equivalent to saying that the covariant functor

$$
\Phi_{\xi}: \operatorname{Mod}_{f, u n, \mathcal{W} \xi}(\mathcal{S}) \rightarrow \operatorname{Mod}_{f}\left(\mathbb{C}\left[\Re_{\xi}, \kappa_{\xi}^{-1}\right]\right)
$$

is an equivalence of categories. Its quasi-inverse is

$$
\rho \mapsto \pi(\xi) \otimes_{\mathbb{C}\left[\Re_{\xi}, \kappa_{\xi}^{-1}\right]} \rho .
$$


It turns out that Theorem4.1 is compatible with parabolic induction, in the following sense. For $P \subset Q \subset F_{0}$ we have $\xi=(P, \delta, t) \in \Xi_{u n}^{Q} \subset \Xi_{\text {un }}$. The R-group of $\mathcal{H}^{Q}=\mathcal{H}\left(\mathcal{R}^{Q}, q\right)$ at $\xi$ is

$$
\mathfrak{R}_{\xi}^{Q}=\left\{r \in \mathfrak{R}_{\xi}: r \text { fixes } t T_{\text {un }}^{Q} \text { pointwise }\right\} .
$$

The cocycle $\kappa_{\xi}^{Q}: \mathfrak{R}_{\xi}^{Q} \times \mathfrak{R}_{\xi}^{Q} \rightarrow \mathbb{C}^{\times}$is the restriction of $\kappa_{\xi}$. By $\mathrm{Opd2}$, Proposition $6.1]$

$$
\operatorname{Ind}_{\mathbb{C}\left[\mathfrak{R}_{\xi}^{Q}, \kappa_{\xi}^{Q}\right]}^{\mathbb{C}\left[\Re_{\xi}, \kappa_{\xi}\right]} \circ \Phi_{\xi}^{* Q}=\Phi_{\xi}^{*} \circ \operatorname{Ind}_{\mathcal{H}^{Q}}^{\mathcal{H}}
$$

as functors $\operatorname{Mod}_{f, u n, \mathcal{W}{ }^{Q} \xi}\left(\mathcal{S}\left(\mathcal{R}^{Q}, q\right)\right) \rightarrow \operatorname{Mod}_{f}\left(\mathbb{C}\left[\Re_{\xi}, \kappa_{\xi}\right]\right)$. This follows from the same argument as for R-groups in the setting of reductive groups, which can be found in [Art, Section 2].

Although it is not needed for our main results, it would be interesting to generalize Theorem 4.1 to non-tempered induction data $\xi \in \Xi \backslash \Xi_{u n}$. We will do that in a weaker sense, only for positive induction data. Sections 5 and 6 do not depend on the remainder of this section.

One approach would be to define the R-group of $\xi=(P, \delta, t)$ to be that of $\xi_{\text {un }}=$ $\left(P, \delta, t|t|^{-1}\right)$. This works out well in most cases, but it is unsatisfactory if the isotropy group $\mathcal{W}_{\xi_{u n}}$ is strictly larger than $\mathcal{W}_{\xi}$. The problem is that $t|t|^{-1}$ is not always a generic point of $\left(T^{P}\right)^{\mathcal{W}_{\xi}}$. To overcome this, we define $\mathcal{M}_{\xi}$ to be the collection of mirrors $M \in \mathcal{M}^{P, \delta}$ that contain not only $t|t|^{-1}$, but the entire connected component of $t|t|^{-1}$ in $\left(T_{u n}^{P}\right)^{\mathcal{W}_{\xi}}$. Notice that all components of $\left(T^{P}\right)^{\mathcal{W}_{\xi}}$ are cosets of a complex subtorus of $T^{P}$. Since $t|t|^{r} \in\left(T^{P}\right)^{\mathcal{W}_{\xi}}$ for all $r \in \mathbb{R}, \mathcal{M}_{\xi}$ is precisely the collection of mirrors whose complexification contains $t$.

Now we define $R_{\xi}, R_{\xi}^{+}$and $\mathfrak{R}_{\xi}$ as in (48) and (49). We note that $\mathfrak{R}_{\xi}$ may differ from $\mathfrak{R}_{\xi_{u n}}$, but that $R_{\xi}=R_{P, \delta, t^{\prime}}$ for almost all $t^{\prime} \in\left(T_{u n}^{P}\right)^{\mathcal{W}_{\xi}}$. For positive induction data there is an analogue of Theorem 4.1 .

Theorem 4.2. Let $\xi=(P, \delta, t) \in \Xi^{+}$.

(a) For $w \in \mathcal{W}_{\xi}$ the intertwiner $\pi(w, \xi)$ is scalar if and only if $w \in W\left(R_{\xi}\right)$.

(b) There exists a 2-cocycle $\kappa_{\xi}$ of $\mathfrak{R}_{\xi}$ such that $\operatorname{End}_{\mathcal{H}}(\pi(\xi))$ is isomorphic to the twisted group algebra $\mathbb{C}\left[\Re_{\xi}, \kappa_{\xi}\right]$.

(c) There exists a unique bijection

$$
\operatorname{Irr}\left(\mathbb{C}\left[\Re_{\xi}, \kappa_{\xi}\right]\right) \rightarrow \operatorname{Irr}_{\mathcal{W} \xi}(\mathcal{H}): \rho \mapsto \pi(\xi, \rho),
$$

and there exist indecomposable direct summands $\pi_{\rho}$ of $\pi(\xi)$, such that $\pi(\xi, \rho)$ is a quotient of $\pi_{\rho}$ and $\pi(\xi) \cong \bigoplus_{\rho} \pi_{\rho} \otimes \rho$ as $\mathcal{H} \otimes \mathbb{C}\left[\Re_{\xi}, \kappa_{\xi}\right]$-modules.

Remark. Part (a) also holds for general $\xi \in \Xi$. Part (d) of Theorem 4.1 does not admit a nice generalization to $\xi \in \Xi^{+}$. One problem is that the category $\operatorname{Mod}_{f}\left(\mathbb{C}\left[\mathfrak{R}_{\xi}, \kappa_{\xi}\right]\right)$ is semisimple, while $\pi(\xi)$ is not always completely reducible. One can try to consider the category of $\mathcal{H}$-representations all whose irreducible subquotients lie in $\operatorname{Irr}_{\mathcal{W}}(\mathcal{H})$, but then one does not get nice formulas for the functors $\Phi_{\xi}$ and $\Phi_{\xi}^{*}$.

Proof. (a) and (b) Since $\xi$ is positive, the operators $\pi(w, \xi)$ with $w \in \mathcal{W}_{\xi}$ span $\operatorname{End}_{\mathcal{H}}(\pi(\xi))$ [Sol3, Theorem 3.3.1]. Let $v \in W\left(R_{\xi}\right)$. The intertwiner $\pi\left(v, P, \delta, t^{\prime}\right)$ is rational in $t^{\prime}$ and by Theorem 4.1,a it is scalar for all $t^{\prime}$ in a Zariski-dense subset of 
$\left(T_{u n}^{P}\right)^{\mathcal{W}_{\xi}}$. Hence $\pi\left(v, P, \delta, t^{\prime}\right)$ is scalar for all $t^{\prime} \in\left(T^{P}\right)^{\mathcal{W}_{\xi}}$, which together with (49) and (18) implies that

$$
\operatorname{End}_{\mathcal{H}}(\pi(\xi))=\operatorname{span}\left\{\pi(w, \xi): w \in \mathfrak{R}_{\xi}\right\}
$$

All the intertwiners $\pi\left(w, P, \delta, t^{\prime}\right)$ depend continuously on $t^{\prime}$, so the type of $\pi\left(P, \delta, t^{\prime}\right)$ as a projective $\mathfrak{R}_{\xi}$-representation is constant on connected components of $\left(T^{P}\right)^{\mathcal{W}_{\xi}}$. Again by Theorem 4.1,a $\left\{\pi(w, \xi): w \in \mathfrak{R}_{\xi}\right\}$ is linearly independent for generic $t^{\prime} \in\left(T_{u n}^{P}\right)^{\mathcal{W}_{\xi}}$, so it is in fact linearly independent for all $t^{\prime} \in\left(T^{P}\right)^{\mathcal{W}_{\xi}}$. Now the multiplication rules for intertwining operators (18) show that $\operatorname{End}_{\mathcal{H}}(\pi(\xi))$ is isomorphic to a twisted group algebra of $\mathfrak{R}_{\xi}$.

(c) Let $A=\operatorname{End}_{\mathbb{C}\left[\Re_{\xi}, \kappa_{\xi}\right]}(\pi(\xi))$ be the bicommutant of $\pi(\xi, \mathcal{H})$ in $\operatorname{End}_{\mathbb{C}}(\pi(\xi))$. There is a canonical bijection

$$
\operatorname{Irr}\left(\mathbb{C}\left[\Re_{\xi}, \kappa_{\xi}\right]\right) \rightarrow \operatorname{Irr}(A): \rho \mapsto \pi_{\rho}
$$

such that $\pi(\xi) \cong \bigoplus_{\rho} \pi_{\rho} \otimes \rho$ as $A \otimes \mathbb{C}\left[\Re_{\xi}, \kappa_{\xi}\right]$-modules. By construction the irreducible $A$-subrepresentations of $\pi(\xi)$ are precisely the indecomposable $\mathcal{H}$-subrepresentations of $\pi(\xi)$. By [Sol3, Proposition 3.1.4] every $\pi_{\rho}$ has a unique irreducible quotient $\mathcal{H}$-representation, say $\pi(\xi, \rho)$, and $\pi(\xi, \rho) \cong \pi\left(\xi, \rho^{\prime}\right)$ if and only if $\pi_{\rho} \cong \pi_{\rho^{\prime}}$ as $\mathcal{H}$ representations. Thus $\rho \mapsto \pi(\xi, \rho)$ sets up a bijection between $\operatorname{Irr}\left(\mathbb{C}\left[\Re_{\xi}, \kappa_{\xi}\right]\right)$ and the equivalence classes of irreducible quotients of $\pi(\xi)$. By Theorem 1.2 the latter collection is none other than $\operatorname{Irr}_{\mathcal{W} \xi}(\mathcal{H})$.

\section{EXtensions of IRREDUCIBLE TEMPERED MODUlES}

In this section we will determine the structure of $\mathcal{S}$ in an infinitesimal neighborhood of a central character $\mathcal{W} \xi$, and we will compute the Ext-groups for irreducible representations of this algebra.

As $\Xi_{u n}$ has the structure of a smooth manifold (with components of different dimensions) we can consider its tangent space $T_{\xi}\left(\Xi_{\text {un }}\right)$ at $\xi$. The isotropy group $\mathcal{W}_{\xi}$ acts linearly on $T_{\xi}\left(\Xi_{u n}\right)$ and for $w \in \mathcal{W}_{\xi}$ we denote the determinant of the corresponding linear map by $\operatorname{det}(w)_{T_{\xi}\left(\Xi_{u n}\right)}$. Since the connected component of $\xi$ in $\Xi_{\text {un }}$ is diffeomorphic to $T_{u n}^{P}$, we have

$$
T_{\xi}\left(\Xi_{u n}\right) \cong \operatorname{Lie}\left(T_{u n}^{P}\right) \cong i \mathfrak{a}^{P} \cong i\left(\mathfrak{a} / \mathfrak{a}_{P}\right) .
$$

Recall the canonical $\mathcal{W}_{\xi}$-representation on $T_{\xi}\left(\Xi_{u n}\right)$, and the decomposition $\mathcal{W}_{\xi}=$ $\mathcal{W}\left(R_{\xi}\right) \rtimes \mathfrak{R}_{\xi}$. By Chevalley's Theorem [Che, Hum the algebra $\mathbb{R}\left[T_{\xi}\left(\Xi_{u n}\right)\right]^{W\left(R_{\xi}\right)}$ of real polynomial invariants on $T_{\xi}\left(\Xi_{u n}\right)$ for the action of the real reflection group $W\left(R_{\xi}\right)$ is itself a free real polynomial algebra on $\operatorname{dim} T_{u n}^{P}$ generators. Let $m_{\xi}$ be its maximal ideal of $W\left(R_{\xi}\right)$-invariant polynomials vanishing at $\xi$. Define

$$
E_{\xi}=m_{\xi} / m_{\xi}^{2} .
$$

Then $E_{\xi}$ carries itself a real representation of $\mathfrak{R}_{\xi}$. Since the $\mathfrak{R}_{\xi}$-invariant ideal $m_{\xi}^{2}$ has finite codimension in $\mathbb{R}\left[T_{\xi}\left(\Xi_{u n}\right)\right]^{W\left(R_{\xi}\right)}$ we can choose a decomposition

$$
\mathbb{R}\left[T_{\xi}\left(\Xi_{\text {un }}\right)\right]^{W\left(R_{\xi}\right)}=\mathbb{R} \oplus E_{\xi} \oplus m_{\xi}^{2}
$$

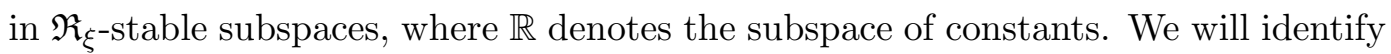
$E_{\xi}$ with this $\mathfrak{R}_{\xi}$-subrepresentation of $\mathbb{R}\left[T_{\xi}\left(\Xi_{u n}\right)\right]^{W\left(R_{\xi}\right)}$. Clearly $E_{\xi}$ generates the ideal $m_{\xi}$. Let $S\left(E_{\xi}\right)=\mathcal{O}\left(E_{\xi}^{*} \otimes_{\mathbb{R}} \mathbb{C}\right)$ be the algebra of complex valued polynomial 
functions on $E_{\xi}^{*}$ and let $\widehat{S\left(E_{\xi}\right)}$ be its formal completion at $0 \in E_{\xi}^{*}$. Notice that (56) induces $\mathfrak{R}_{\xi}$-equivariant isomorphisms

$$
\mathcal{O}\left(T_{\xi}\left(\Xi_{u n}\right)\right)^{W\left(R_{\xi}\right)} \cong S\left(E_{\xi}\right) \text { and } \quad \mathcal{F}_{\xi}^{W\left(R_{\xi}\right)} \cong \widehat{S\left(E_{\xi}\right)}
$$

Let $\kappa_{\xi}: \mathfrak{R}_{\xi} \times \mathfrak{R}_{\xi} \rightarrow \mathbb{C}^{\times}$be the cocycle from Theorem 4.1,b and let $\mathfrak{R}_{\xi}^{*}$ be a Schur extension of $\mathfrak{R}_{\xi}$. This object is also known as a representation group [CuRe, Section 53] and is characterized by the property that equivalence classes of irreducible projective $\mathfrak{R}_{\xi}$-representations are in natural bijection with equivalence classes of (linear) $\mathfrak{R}_{\xi}^{*}$-representations. There is a unique central idempotent $p_{\xi} \in \mathbb{C}\left[\mathfrak{R}_{\xi}^{*}\right]$ such that

$$
\mathbb{C}\left[\Re_{\xi}, \kappa_{\xi}^{-1}\right] \cong p_{\xi} \mathbb{C}\left[\Re_{\xi}^{*}\right]
$$

as algebras and as $\mathbb{C}\left[\mathfrak{R}_{\xi}^{*}\right]$-bimodules. Then $p_{\xi}^{*} \mathbb{C}\left[\mathfrak{R}_{\xi}^{*}\right]=\mathbb{C}\left[\mathfrak{R}_{\xi}, \kappa_{\xi}\right]$. With these notations we can formulate a strengthening of Theorem 4.1 .

Theorem 5.1. The Fréchet algebras $\widehat{\mathcal{S}}_{\mathcal{W} \xi}$ and $p_{\xi}\left(\widehat{S\left(E_{\xi}\right)} \rtimes \mathfrak{R}_{\xi}^{*}\right)$ are Morita equivalent. There are equivalences of exact categories

$$
\operatorname{Mod}_{b o r}^{\mathcal{W} \xi \text { tor }}(\mathcal{S}) \cong \operatorname{Mod}_{\text {bor }}\left(\widehat{\mathcal{S}}_{\mathcal{W} \xi}\right) \cong \operatorname{Mod}_{\text {bor }}\left(p_{\xi}\left(\widehat{S\left(E_{\xi}\right)} \rtimes \mathfrak{R}_{\xi}^{*}\right)\right)
$$

and similarly for Fréchet modules.

Proof. Write $\xi=(P, \delta, t)$. By (23) and (24) we may replace $\mathcal{S}$ by

$$
\left(C^{\infty}\left(T_{u n}^{P}\right) \otimes \operatorname{End}_{\mathbb{C}}\left(\mathbb{C}\left[W^{P}\right] \otimes V_{\delta}\right)\right)^{\mathcal{W}_{P, \delta}} .
$$

Let $U_{\xi} \subset T_{u n}^{P}$ be a nonempty open ball around $t$ such that

$$
\overline{U_{\xi}} \cap w \overline{U_{\xi}}=\left\{\begin{array}{lll}
\overline{U_{\xi}} & \text { if } \quad w \in \mathcal{W}_{\xi} \\
\emptyset & \text { if } \quad w \in \mathcal{W}_{P, \delta} \backslash \mathcal{W}_{\xi} .
\end{array}\right.
$$

The localization of (58) at $U_{\xi}$ is

$$
\begin{array}{r}
\left.C^{\infty}\left(U_{\xi}\right)^{\mathcal{W}_{\xi}} \otimes_{C^{\infty}\left(T_{u n}^{P}\right)}\right)^{\mathcal{W}_{P, \delta}}\left(C^{\infty}\left(T_{u n}^{P}\right) \otimes \operatorname{End}_{\mathbb{C}}\left(\mathbb{C}\left[W^{P}\right] \otimes V_{\delta}\right)\right)^{\mathcal{W}_{P, \delta}} \\
\cong\left(C^{\infty}\left(U_{\xi}\right) \otimes \operatorname{End}_{\mathbb{C}}\left(\mathbb{C}\left[W^{P}\right] \otimes V_{\delta}\right)\right)^{\mathcal{W}_{\xi}} .
\end{array}
$$

The action of $\mathcal{W}_{\xi}$ on $C^{\infty}\left(U_{\xi}\right) \otimes \operatorname{End}_{\mathbb{C}}\left(\mathbb{C}\left[W^{P}\right] \otimes V_{\delta}\right)$ is still defined by (22), so for $w \in \mathcal{W}_{\xi}$ and $t^{\prime} \in U_{\xi}$

$$
(w \cdot f)\left(t^{\prime}\right)=\pi\left(w, P, \delta, w^{-1} t^{\prime}\right) f\left(w^{-1} t^{\prime}\right) \pi\left(w, P, \delta, w^{-1} t^{\prime}\right)^{-1} .
$$

Let $V_{\xi}$ be the vector space $\mathbb{C}\left[W^{P}\right] \otimes V_{\delta}$ endowed with the $\mathcal{H}$-representation $\pi(\xi)$. It is also a projective $\mathcal{W}_{\xi}$-representation, so we can define a $\mathcal{W}_{\xi}$-action on $C^{\infty}\left(U_{\xi}\right) \otimes$ $\operatorname{End}_{\mathbb{C}}\left(V_{\xi}\right)$ by

$$
(w \cdot f)\left(t^{\prime}\right)=\pi(w, \xi) f\left(w^{-1} t^{\prime}\right) \pi(w, \xi)^{-1} .
$$

The difference between (60) and (61) is that $\pi\left(w, P, \delta, w^{-1} t^{\prime}\right)$ depends on $t^{\prime}$, while $\pi(w, \xi)$ does not. Since $U_{\xi}$ is $\mathcal{W}_{\xi}$-equivariantly contractible to $t$, we are in the right position to apply [Sol1, Lemma 7]. Its proof, and in particular [Sol1, (20)] shows that the algebra (59), with the $\mathcal{W}_{\xi}$-action (60), is isomorphic to

$$
\left(C^{\infty}\left(U_{\xi}\right) \otimes \operatorname{End}_{\mathbb{C}}\left(V_{\xi}\right)\right)^{\mathcal{W}_{\xi}}
$$


with respect to the $\mathcal{W}_{\xi}$-action (61). By Theorem 4.1. a the elements of $W\left(R_{\xi}\right)$ act trivially on $\operatorname{End}_{\mathbb{C}}\left(V_{\xi}\right)$, but nontrivially on $C^{\infty}\left(U_{\xi}\right)$. Moreover by (49) $W\left(R_{\xi}\right)$ is normal in $\mathcal{W}_{\xi}$ and $\mathcal{W}_{\xi} / W\left(R_{\xi}\right) \cong \mathfrak{R}_{\xi}$, so we can rewrite (62) as

$$
\left(C^{\infty}\left(U_{\xi}\right)^{W\left(R_{\xi}\right)} \otimes \operatorname{End}_{\mathbb{C}}\left(V_{\xi}\right)\right)^{\Re_{\xi}}
$$

The formal completion of (63) at $\xi$ is

$$
\mathcal{F}_{\xi}^{\mathcal{W}_{\xi}} \widehat{\otimes}_{C \infty\left(U_{\xi}\right)^{\mathcal{W}_{\xi}}}\left(C^{\infty}\left(U_{\xi}\right)^{W\left(R_{\xi}\right)} \otimes \operatorname{End}_{\mathbb{C}}\left(V_{\xi}\right)\right)^{\mathfrak{R}_{\xi}}
$$

By Lemma 2.2, a the latter is isomorphic to

$$
\left(\left(C^{\infty}\left(U_{\xi}\right)^{W\left(R_{\xi}\right)} / \overline{\left(m_{\xi}^{\mathcal{W}_{\xi}, \infty} C^{\infty}\left(U_{\xi}\right)^{W\left(R_{\xi}\right)}\right.}\right) \otimes \operatorname{End}_{\mathbb{C}}\left(V_{\xi}\right)\right)^{\mathfrak{R}_{\xi}},
$$

where $m_{\xi}^{\mathcal{W}_{\xi}, \infty} \subset C^{\infty}\left(U_{\xi}\right)^{\mathcal{W}_{\xi}}$ denotes the ideal of flat functions at $\xi$. We claim that

$$
\overline{m_{\xi}^{\mathcal{W}_{\xi}, \infty} C^{\infty}\left(U_{\xi}\right)^{W\left(R_{\xi}\right)}}=m_{\xi}^{W\left(R_{\xi}\right), \infty},
$$

the ideal of flat functions in $C^{\infty}\left(U_{\xi}\right)^{W\left(R_{\xi}\right)}$. By Whitney's spectral theorem the right hand side of (66) is closed, so it contains the left hand side. Let us prove the opposite inclusion. By the finiteness of $\mathfrak{R}_{\xi}$ the ring of $W\left(R_{\xi}\right)$-invariant polynomials $\mathcal{O}\left(T_{\xi}\left(\Xi_{u n}\right)\right)^{W\left(R_{\xi}\right)}$ is finitely generated as a module over $\mathcal{O}\left(T_{\xi}\left(\Xi_{u n}\right)\right)^{\mathcal{W}_{\xi}}$. Since $\mathcal{O}\left(T_{\xi}\left(\Xi_{u n}\right)\right)^{\mathcal{W}_{\xi}}$ is Noetherian ring, there exists a finite presentation

$$
\left(\mathcal{O}\left(T_{\xi}\left(\Xi_{u n}\right)\right)^{\mathcal{W}_{\xi}}\right)^{n_{2}} \stackrel{A}{\longrightarrow}\left(\mathcal{O}\left(T_{\xi}\left(\Xi_{u n}\right)\right)^{\mathcal{W}_{\xi}}\right)^{n_{1}} \stackrel{P}{\longrightarrow} \mathcal{O}\left(T_{\xi}\left(\Xi_{u n}\right)\right)^{W\left(R_{\xi}\right)} \rightarrow 0 .
$$

Let $P_{i} \in \mathcal{O}\left(T_{\xi}\left(\Xi_{u n}\right)\right)^{W\left(R_{\xi}\right)}$ be the image of the $i$-th basis vector under $P$. Then $A$ is an $n_{1} \times n_{2}$-matrix with entries in $\mathcal{O}\left(T_{\xi}\left(\Xi_{u n}\right)\right)^{\mathcal{W}_{\xi}}$, expressing the relations be-

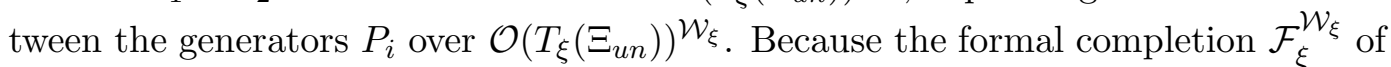
$\mathcal{O}\left(T_{\xi}\left(\Xi_{u n}\right)\right)^{\mathcal{W}_{\xi}}$ at $\xi$ is flat over $\mathcal{O}\left(T_{\xi}\left(\Xi_{u n}\right)\right)^{\mathcal{W}_{\xi}}$, the relations module of the generators $P_{i}$ is also given by the matrix $A$ when working over the formal power series ring $\mathcal{F}_{\xi}^{\mathcal{W}_{\xi}}$. In addition one easily checks that

$$
\mathcal{F}_{\xi}^{\mathcal{\mathcal { W } _ { \xi }}} \otimes_{\mathcal{O}\left(T_{\xi}\left(\Xi_{u n}\right)\right)^{\mathcal{W} \xi}} \mathcal{O}\left(T_{\xi}\left(\Xi_{u n}\right)\right)^{W\left(R_{\xi}\right)}=\mathcal{F}_{\xi}^{W\left(R_{\xi}\right)}
$$

Now let $f \in m_{\xi}^{W\left(R_{\xi}\right), \infty}$. By an argument of Poénaru [Poe, p.106] the $P_{i}$ also generate $C^{\infty}\left(U_{\xi}\right)^{W\left(R_{\xi}\right)}$ as a module over $C^{\infty}\left(U_{\xi}\right)^{\mathcal{W}_{\xi}}$, so we may write $f=\sum_{i=1}^{n_{1}} \phi_{i} P_{i}$ with $\phi=\left(\phi_{i}\right)_{i=1}^{n_{1}} \in\left(C^{\infty}\left(U_{\xi}\right)^{\mathcal{W}_{\xi}}\right)^{n_{1}}$. By applying the Taylor series homomorphism $\tau_{\xi}$ at $\xi$ we get $0=\sum_{i=1}^{n_{1}} \tau_{\xi}\left(\phi_{i}\right) P_{i}$. It follows from (67) and the remarks just above that there exists a $\widehat{\psi} \in\left(\mathcal{F}_{\xi}^{\mathcal{W}_{\xi}}\right)^{n_{2}}$ such that $\tau_{\xi}(\phi)=A(\widehat{\psi})$. By Borel's Theorem there exist $\psi \in\left(C^{\infty}\left(U_{\xi}\right)^{\mathcal{W}_{\xi}}\right)^{n_{2}}$ such that $\tau_{\xi}(\psi)=\widehat{\psi}$. Writing $\phi^{0}:=A(\psi) \in\left(C^{\infty}\left(U_{\xi}\right)^{\mathcal{W}_{\xi}}\right)^{n_{1}}$, we obtain

$$
\begin{aligned}
& \phi_{i}-\phi_{i}^{0} \in m_{\xi}^{W\left(R_{\xi}\right), \infty} \cap C^{\infty}\left(U_{\xi}\right)^{\mathcal{W}_{\xi}}=m_{\xi}^{\mathcal{W}_{\xi}, \infty}, \\
& f=\sum_{i=1}^{n_{1}} \phi_{i} P_{i}=\sum_{i=1}^{n_{1}}\left(\phi_{i}-\phi_{i}^{0}\right) P_{i} \in m_{\xi}^{\mathcal{W}_{\xi}, \infty} C^{\infty}\left(U_{\xi}\right)^{W\left(R_{\xi}\right)} .
\end{aligned}
$$

This proves (66), from which we conclude that (65) is isomorphic to

$$
\left(\mathcal{F}_{\xi}^{W\left(R_{\xi}\right)} \otimes \operatorname{End}_{\mathbb{C}}\left(V_{\xi}\right)\right)^{\Re_{\xi}} .
$$


For compatibility with Section 3 we put $M=E_{\xi}^{*} \otimes_{\mathbb{R}} \mathbb{C}, m=0$ and $G=G_{m}=\mathfrak{R}_{\xi}^{*}$. By Theorem 4.1,c every irreducible representation of $\mathbb{C}\left[\Re_{\xi}, \kappa_{\xi}\right]=p_{\xi}^{*} \mathbb{C}\left[\Re_{\xi}^{*}\right]$ appears at least once in $V_{\xi}$, so $V_{\xi}$ contains precisely the same irreducible $\mathfrak{R}_{\xi}^{*}$-representations as the right regular representation of $\mathfrak{R}_{\xi}^{*}$ on $\mathbb{C}\left[\mathfrak{R}_{\xi}, \kappa_{\xi}^{-1}\right]=p_{\xi} \mathbb{C}\left[\mathfrak{R}_{\xi}^{*}\right]$. Now parts (a) and (d) of Theorem 3.2 say that (68) is Morita equivalent to

$$
\left(\widehat{S\left(E_{\xi}\right)} \otimes \operatorname{End}_{\mathbb{C}}\left(p_{\xi} \mathbb{C}\left[\Re_{\xi}^{*}\right]\right)\right)^{\mathfrak{R}_{\xi}^{*}}
$$

Because $p_{\xi}$ is central and by Lemma 3.1, (69) is isomorphic to

$$
\widehat{S\left(E_{\xi}\right)} \rtimes p_{\xi} \mathbb{C}\left[\mathfrak{R}_{\xi}^{*}\right]=p_{\xi}\left(\widehat{S\left(E_{\xi}\right)} \rtimes \mathfrak{R}_{\xi}^{*}\right) .
$$

This establishes the required Morita equivalence. Since the bimodules in the proof of Theorem 3.2 a are bornologically projective, this Morita equivalence is an exact functor. That and parts (c) and (f) of Lemma 2.2 yield the equivalences of exact categories.

The above proof and Theorem 3.2 show that the Morita bimodules are

$$
\left(\widehat{S\left(E_{\xi}\right)} \otimes \operatorname{Hom}_{\mathbb{C}}\left(\pi(\xi), p_{\xi} \mathbb{C}\left[\Re_{\xi}^{*}\right]\right)\right)^{\mathfrak{R}_{\xi}^{*}} \text { and }\left(\widehat{S\left(E_{\xi}\right)} \otimes \operatorname{Hom}_{\mathbb{C}}\left(p_{\xi} \mathbb{C}\left[\Re_{\xi}^{*}\right], \pi(\xi)\right)\right)^{\mathfrak{R}_{\xi}^{*}}
$$

where $\mathbb{C}\left[\mathfrak{R}_{\xi}^{*}\right]$ is considered as the right regular representation of $\mathfrak{R}_{\xi}^{*}$. The equivalence of categories maps $\pi \in \operatorname{Mod}_{\mathcal{W} \xi}(\mathcal{S})$ to the $\widehat{S\left(E_{\xi}\right)} \rtimes \mathfrak{R}_{\xi}^{*}$-module

$$
\operatorname{Hom}_{\mathbb{C}}\left(\pi(\xi), p_{\xi} \mathbb{C}\left[\mathfrak{R}_{\xi}^{*}\right]\right)^{\mathfrak{R}_{\xi}^{*}} \underset{\operatorname{End}_{\mathbb{C}}(\pi(\xi))^{\mathfrak{R}_{\xi}^{*}}}{\otimes} \pi=p_{\xi} \operatorname{Hom}_{\mathbb{C}\left[\mathfrak{R}_{\xi}^{*}\right]}\left(\pi(\xi), \mathbb{C}\left[\mathfrak{R}_{\xi}^{*}\right]\right) \underset{\operatorname{End}_{\mathbb{C}\left[\mathfrak{R}_{\xi}^{*}\right]}(\pi(\xi))}{\otimes} \pi .
$$

By construction $\operatorname{End}_{\mathbb{C}\left[\Re_{\xi}^{*}\right]}(\pi(\xi))=\pi(\xi)(\mathcal{H})$, while $\operatorname{Hom}_{\mathbb{C}\left[\Re_{\xi}^{*}\right]}\left(\pi(\xi), \mathbb{C}\left[\Re_{\xi}^{*}\right]\right)$ is isomorphic to the dual space of $\pi(\xi)$ via the map $\mathbb{C}\left[\mathfrak{R}_{\xi}^{*}\right] \rightarrow \mathbb{C}$ defined by evaluation at 1 . Hence the above module simplifies to

$$
p_{\xi} \pi(\xi)^{*} \otimes_{\mathcal{H}} \pi=\pi(\xi)^{*} \otimes_{\mathcal{H}} \pi=\operatorname{Hom}_{\mathcal{H}}(\pi(\xi), \pi) .
$$

Conversely, consider a $\widehat{S\left(E_{\xi}\right)} \rtimes p_{\xi} \mathbb{C}\left[\Re_{\xi}^{*}\right]$-module $\rho$ with central character $0 \in E_{\xi}^{*}$. The Morita equivalence sends it to

$$
\begin{array}{r}
\operatorname{Hom}_{\mathbb{C}}\left(p_{\xi} \mathbb{C}\left[\Re_{\xi}^{*}\right], \pi(\xi)\right)^{\mathfrak{R}_{\xi}^{*}} \underset{\operatorname{End}_{\mathbb{C}}\left(p_{\xi} \mathbb{C}\left[\Re_{\xi}^{*}\right]\right)^{\mathfrak{R}_{\xi}^{*}}}{\otimes} \rho=p_{\xi}^{*} \operatorname{Hom}_{\mathbb{C}\left[\Re_{\xi}^{*}\right]}\left(p_{\xi} \mathbb{C}\left[\mathfrak{R}_{\xi}^{*}\right], \pi(\xi)\right) \underset{p_{\xi} \mathbb{C}\left[\mathfrak{R}_{\xi}^{*}\right]}{\otimes} \rho \\
=p_{\xi}^{*} \pi(\xi) \underset{p_{\xi} \mathbb{C}\left[\mathfrak{R}_{\xi}^{*}\right]}{\rho}=\pi(\xi) \underset{\mathbb{C}\left[\mathfrak{R}_{\xi}, \kappa_{\xi}^{-1}\right]}{\otimes} \rho .
\end{array}
$$

Together with (51) and (52) this confirms that Theorem 5.1 generalizes the covariant version of Theorem 4.1, d.

If $\pi^{\prime} \in \operatorname{Mod}_{f, u n, \mathcal{W} \xi}(\mathcal{S})$, then by (50)

$$
\Phi_{\xi}^{*}(\pi) \otimes \Phi_{\xi}\left(\pi^{\prime}\right) \text { is a representation of } \mathbb{C}\left[\Re_{\xi}, 1\right],
$$

that is, a (linear) representation of the group $\mathfrak{R}_{\xi}$.

Theorem 5.2. Let $\pi, \pi^{\prime}$ be finite dimensional unitary $\mathcal{S}$-modules with central character $\mathcal{W} \xi$. Let $\Phi_{\xi}^{*}(\pi)=\operatorname{Hom}_{\mathcal{H}}(\pi, \pi(\xi)) \in \operatorname{Mod}\left(\mathbb{C}\left[\Re_{\xi}, \kappa_{\xi}\right]\right)$ and $\Phi_{\xi}\left(\pi^{\prime}\right)=\operatorname{Hom}_{\mathcal{H}}\left(\pi(\xi), \pi^{\prime}\right) \in \operatorname{Mod}\left(\mathbb{C}\left[\Re_{\xi}, \kappa_{\xi}^{-1}\right]\right)$ be as in Theorem 4.1. Then

$$
\operatorname{Ext}_{\mathcal{H}}^{n}\left(\pi, \pi^{\prime}\right) \cong\left(\Phi_{\xi}^{*}(\pi) \otimes_{\mathbb{C}} \Phi_{\xi}\left(\pi^{\prime}\right) \otimes_{\mathbb{R}} \bigwedge^{n} E_{\xi}^{*}\right)^{\Re_{\xi}} .
$$


For $\pi=\pi^{\prime}$ the Yoneda-product on $\operatorname{Ext}_{\mathcal{H}}^{*}(\pi, \pi)$ corresponds to the usual product on $\left(\operatorname{End}_{\mathbb{C}}\left(\Phi_{\xi}(\pi)\right) \otimes_{\mathbb{R}} \bigwedge^{*} E_{\xi}^{*}\right)^{\mathfrak{R}_{\xi}}$.

Proof. Our strategy is to consider $\pi$ and $\pi^{\prime}$ as modules over various algebras $A$, such that the extension groups $\operatorname{Ext}_{A}^{n}\left(\pi, \pi^{\prime}\right)$ for different $A$ 's are all isomorphic. By OpSo1, Corollary 3.7.b]

$$
\operatorname{Ext}_{\mathcal{H}}^{n}\left(\pi, \pi^{\prime}\right) \cong \operatorname{Ext}_{\mathcal{S}}^{n}\left(\pi, \pi^{\prime}\right) \quad n \in \mathbb{Z}_{\geq 0} .
$$

Since $\pi$ and $\pi^{\prime}$ have finite dimension, it does not matter whether we calculate their Ext-groups in the category of algebraic $\mathcal{S}$-modules or in any of the categories of topological $\mathcal{S}$-modules studied in Section 2 .

From (74) and exactness of localization we see that the first steps of the proof of Theorem [3.2, up to (63), preserve the Ext-groups. For the computation of $\operatorname{Ext}_{\mathcal{S}}^{n}\left(\pi, \pi^{\prime}\right)$, Corollary 2.6 allows us to replace $\mathcal{S}$ by its formal completion $\widehat{\mathcal{S}}_{\mathcal{W} \xi} \cong$ $\mathcal{F}_{\xi}^{\mathcal{W}} \widehat{\otimes}_{\mathcal{Z}} \mathcal{S}$ at the central character $\mathcal{W} \xi$. This brings us to (64), which is Morita equivalent to (70). Under these transformations $\pi$ corresponds to $\Phi_{\xi}(\pi)$, regarded as a representation of (70) or of $\widehat{S\left(E_{\xi}\right)} \rtimes \Re_{\xi}^{*}$-representation via the evaluation at 0 . Because (70) is a direct summand of $\widehat{S\left(E_{\xi}\right)} \rtimes \mathfrak{R}_{\xi}^{*}$, we can consider the Ext-groups of $\Phi_{\xi}(\pi)$ and $\Phi_{\xi}\left(\pi^{\prime}\right)$ just as well with respect to $\widehat{S\left(E_{\xi}\right)} \rtimes \mathfrak{R}_{\xi}^{*}$. Therefore

$$
\operatorname{Ext}_{\mathcal{H}}^{n}\left(\pi, \pi^{\prime}\right) \cong \operatorname{Ext}_{\overparen{S\left(E_{\xi}\right)} \rtimes \Re_{\xi}^{*}}^{n}\left(\Phi_{\xi}(\pi), \Phi_{\xi}\left(\pi^{\prime}\right)\right),
$$

and we can apply Theorem 3.3. Here $\mathcal{F}_{G m}=\mathcal{F}_{m}=\widehat{S\left(E_{\xi}\right)}$ and $\bigwedge_{\mathbb{C}}^{n} T_{m}(M) \cong$ $\bigwedge_{\mathbb{R}}^{n} E_{\xi}^{*} \otimes_{\mathbb{R}} \mathbb{C}$, so by Theorem 3.3 ,a

$$
\operatorname{Ext}_{\mathcal{H}}^{n}\left(\pi, \pi^{\prime}\right) \cong\left(\Phi_{\xi}^{*}(\pi) \otimes_{\mathbb{C}} \Phi_{\xi}\left(\pi^{\prime}\right) \otimes_{\mathbb{R}} \bigwedge^{n} E_{\xi}^{*}\right)^{\mathfrak{R}_{\xi}^{*}} .
$$

Moreover $\Phi_{\xi}^{*}(\pi) \otimes_{\mathbb{C}} \Phi_{\xi}\left(\pi^{\prime}\right)$ is a $\mathfrak{R}_{\xi^{-}}$-representation, so the entire action factors through $\mathfrak{R}_{\xi}^{*} \rightarrow \mathfrak{R}_{\xi}$. Thus we may just as well use $\mathfrak{R}_{\xi}$ to determine the invariants in (76)). The Yoneda product is described by Theorem 3.3. b.

\section{The Euler-Poincaré Pairing}

Let $\pi, \pi^{\prime} \in \operatorname{Mod}_{f}(\mathcal{H})$. Their Euler-Poincaré pairing is defined as

$$
E P_{\mathcal{H}}\left(\pi, \pi^{\prime}\right)=\sum_{n \geq 0}(-1)^{n} \operatorname{dim}_{\mathbb{C}} \operatorname{Ext}_{\mathcal{H}}^{n}\left(\pi, \pi^{\prime}\right)
$$

Since $\mathcal{H}$ is Noetherian and has finite cohomological dimension OpSo1, Proposition 2.4], this pairing is well-defined. By the main result of $\mathrm{OpSo1}$, for $\pi, \pi^{\prime} \in \operatorname{Mod}_{f}(\mathcal{S})$

$$
E P_{\mathcal{S}}\left(\pi, \pi^{\prime}\right):=\sum_{n \geq 0}(-1)^{n} \operatorname{dim}_{\mathbb{C}} \operatorname{Ext}_{\mathcal{S}}^{n}\left(\pi, \pi^{\prime}\right) \quad \text { equals } \quad E P_{\mathcal{H}}\left(\pi, \pi^{\prime}\right) .
$$

It is clear from the definition (77) that $E P_{\mathcal{H}}\left(\pi, \pi^{\prime}\right)=0$ if $\pi$ and $\pi^{\prime}$ admit different $Z(\mathcal{H})$-characters. With (78) we can strengthen this to $E P_{\mathcal{H}}\left(\pi, \pi^{\prime}\right)=0$ whenever $\pi$ and $\pi^{\prime}$ admit different $Z(\mathcal{S})$-characters. This is really stronger, because $Z(\mathcal{S})$ is larger than the closure of $Z(\mathcal{H})$ in $\mathcal{S}$. By (24) any discrete series representation $\delta$ is projective in $\operatorname{Mod}_{f}(\mathcal{S})$, so

$$
E P_{\mathcal{S}}\left(\delta, \pi^{\prime}\right)=\operatorname{dim}_{\mathbb{C}} \operatorname{Hom}_{\mathcal{S}}\left(\delta, \pi^{\prime}\right) \text { for all } \pi^{\prime} \in \operatorname{Mod}_{f}(\mathcal{S}) .
$$


Together with (78) this shows that $\delta$ also behaves like a projective $\mathcal{H}$-representation for the Euler-Poincaré pairing (over $\mathcal{H}$ ) of tempered modules, something that is completely unclear from (77).

For $\epsilon \in \mathbb{R}$ let $q^{\epsilon}$ be the parameter function $q^{\epsilon}(w)=q(w)^{\epsilon}$. For every $\epsilon$ we have the affine Hecke algebra $\mathcal{H}\left(\mathcal{R}, q^{\epsilon}\right)$ and its Schwartz completion $\mathcal{S}\left(\mathcal{R}, q^{\epsilon}\right)$. We note that $\mathcal{H}\left(\mathcal{R}, q^{0}\right)=\mathbb{C}[W]$ is the group algebra of $W$ and that $\mathcal{S}\left(\mathcal{R}, q^{0}\right)=\mathcal{S}(W)$ is the Schwartz algebra of rapidly decreasing functions on $W$. The intuitive idea is that these algebras depend continuously on $\epsilon$. We will use this in the form of the following rather technical result.

Theorem 6.1. SSol3, Corollary 4.2.2]

For $\epsilon \in[-1,1]$ there exists a family of additive functors

$$
\begin{aligned}
& \tilde{\sigma}_{\epsilon}: \operatorname{Mod}_{f}(\mathcal{H}(\mathcal{R}, q)) \rightarrow \operatorname{Mod}_{f}\left(\mathcal{H}\left(\mathcal{R}, q^{\epsilon}\right)\right), \\
& \tilde{\sigma}_{\epsilon}(\pi, V)=\left(\pi_{\epsilon}, V\right) .
\end{aligned}
$$

with the properties

(1) the map

$$
[-1,1] \rightarrow \text { End } V: \epsilon \mapsto \pi_{\epsilon}\left(N_{w}\right)
$$

is analytic for any $w \in W$,

(2) $\tilde{\sigma}_{\epsilon}$ is a bijection if $\epsilon \neq 0$,

(3) $\tilde{\sigma}_{\epsilon}$ preserves unitarity,

(4) $\tilde{\sigma}_{\epsilon}$ preserves temperedness if $\epsilon \geq 0$,

(5) $\tilde{\sigma}_{\epsilon}$ preserves the discrete series if $\epsilon>0$.

Theorem 6.2. (a) For all $\pi, \pi^{\prime} \in \operatorname{Mod}_{f}(\mathcal{H})$ and $\epsilon \in[0,1]$ :

$$
E P_{\mathcal{H}\left(\mathcal{R}, q^{\epsilon}\right)}\left(\tilde{\sigma}_{\epsilon}(\pi), \tilde{\sigma}_{\epsilon}\left(\pi^{\prime}\right)\right)=E P_{\mathcal{H}}\left(\pi, \pi^{\prime}\right) .
$$

(b) The pairing $E P_{\mathcal{H}}$ is symmetric and positive semidefinite.

(c) Suppose $P \subset F_{0}, \mathbb{R} P \neq \mathfrak{a}^{*}$ and $V \in \operatorname{Mod}_{f}\left(\mathcal{H}^{P}\right)$. Then $\operatorname{Ind}_{\mathcal{H}^{P}}^{\mathcal{H}}(V)$ lies in the radical of $E P_{\mathcal{H}}$.

Proof. See Proposition 3.4 and Theorem 3.5 of OpSo1. We note that the symmetry of $E P_{\mathcal{H}}$ is not automatic, it is proved via part (a) for $\epsilon=0$ and a more detailed study of $E P_{W}=E P_{\mathcal{H}\left(\mathcal{R}, q^{0}\right)}$ OpSo1, Theorem 3.2].

The pairing $E P_{\mathcal{H}}$ extends naturally to a Hermitian form on $G_{\mathbb{C}}(\mathcal{H})$, say complex linear in the second argument. By Theorem 6.2. c it factors through the quotient

$$
\operatorname{Ell}(\mathcal{H}):=G_{\mathbb{C}}(\mathcal{H}) / \sum_{P \subset F_{0}, \mathbb{R} P \neq \mathfrak{a}^{*}} \operatorname{Ind}_{\mathcal{H}^{P}}^{\mathcal{H}}\left(G_{\mathbb{C}}\left(\mathcal{H}^{P}\right)\right)
$$

Following $\operatorname{Opd} 2$ we call $\operatorname{Ell}(\mathcal{H})$ the space of elliptic characters of $\mathcal{H}$. Notice that $\operatorname{Ell}(\mathcal{H})=0$ and $E P_{\mathcal{H}}=0$ if the root datum $\mathcal{R}$ is not semisimple.

Lemma 6.3. The composite map $G_{\mathbb{C}}(\mathcal{S}) \rightarrow G_{\mathbb{C}}(\mathcal{H}) \rightarrow \operatorname{Ell}(\mathcal{H})$ is surjective.

Proof. We may and will assume that $\mathcal{R}$ is semisimple. We have to show that every irreducible $\mathcal{H}$-representation $\pi$ can be written as a linear combination of tempered representations and of representations induced from proper parabolic subalgebras. Recall [Sol3, Section 2.2] that a Langlands datum is a triple $(P, \sigma, t)$ such that

- $P \subset F_{0}$ and $\sigma$ is an irreducible tempered $\mathcal{H}_{P}$-representation;

- $t \in T^{P}$ and $|t| \in T^{P++}$. 
The Langlands classification [Sol3, Theorem 2.2.4] says that the $\mathcal{H}$-representation $\pi(P, \sigma, t)$ has a unique irreducible quotient $L(P, \sigma, t)$ and that there is (up to equivalence) a unique Langlands datum such that $L(P, \sigma, t) \cong \pi$.

If $P=F_{0}$, then $T^{P}=\{1\}$ because $\mathcal{R}$ is semisimple. So $\mathcal{H}_{P}=\mathcal{H}^{P}=\mathcal{H}$ and $\pi \cong \sigma$, which by definition is tempered.

Therefore we may suppose that $P \neq F_{0}$. Since $\pi(P, \sigma, t)$ is induced from $\mathcal{H}^{P}$, we have $\pi=L(P, \sigma, t)-\pi(P, \sigma, t)$ in $\operatorname{Ell}(\mathcal{H})$. By [Sol3, Lemma 2.2.6.b]

$$
\pi(P, \sigma, t)-L(P, \sigma, t) \in G_{\mathbb{C}}(\mathcal{H})
$$

is a sum of representations $L(Q, \tau, s)$ with $P \subset Q$ and $\left\|c c_{P}(\sigma)\right\|<\left\|c c_{Q}(\tau)\right\|$, where $c_{P}(\sigma)$ is as in (19). Given the central character of $\pi$ (an element of $T / W_{0}$ ), there are only finitely many possibilities for $c_{P}(\sigma)$. Hence we can deal with the representations $L(Q, \tau, s)$ via an inductive argument.

From [Sol3, Lemma 4.2.3.a] we know that $\tilde{\sigma}_{0}: \operatorname{Mod}_{f}(\mathcal{H}) \rightarrow \operatorname{Mod}_{f}(W)$ commutes with parabolic induction, so it induces a linear map

$$
\sigma_{\mathrm{Ell}}: \operatorname{Ell}(\mathcal{H}) \rightarrow \operatorname{Ell}(W)=\operatorname{Ell}\left(\mathcal{H}\left(\mathcal{R}, q^{0}\right)\right) .
$$

Theorem 6.4. The pairings $E P_{\mathcal{H}}$ and $E P_{W}$ induce Hermitian inner products on respectively $\operatorname{Ell}(\mathcal{H})$ and $\operatorname{Ell}(W)$, and the map $\sigma_{\mathrm{Ell}}$ is an isometric bijection.

Proof. By Lemma 6.3 and [Sol3, (3.37)], $\sigma_{\text {Ell }}$ is a linear bijection. According to OpSo1, Theorem 3.2.b] $E P_{W}$ induces a Hermitian inner product on $\operatorname{Ell}(W)$, and by Theorem $6.2 \sigma_{\mathrm{Ell}}$ is an isometry. Therefore the sesquilinear form on $\operatorname{Ell}(\mathcal{H})$ induced by $E P_{\mathcal{H}}$ is also a Hermitian inner product.

6.1. Arthur's formula. In this subsection we prove Arthur's formula for the EulerPoincaré pairing (77) for tempered representations of affine Hecke algebras (2).

Recall the setup of Theorem 5.2. The expression $\operatorname{det}(1-w)_{T_{\xi}\left(\Xi_{u n}\right)}=\operatorname{det}(1-w)_{\mathfrak{a}^{P}}$ is analogous to the "Weyl measure" in equation (3) and to $d(r)$ in equation (5). Notice that

$$
\operatorname{det}(1-w)_{T_{\xi}\left(\Xi_{u n}\right)} \geq 0
$$

because the tangent space of $\Xi_{u n}$ at $\xi$ is a real representation of the finite group $\mathcal{W}_{\xi}$. Clearly $\operatorname{det}(1-w)_{T_{\xi}\left(\Xi_{u n}\right)} \neq 0$ if and only if $w$ acts without fixed points on $T_{\xi}\left(\Xi_{u n}\right) \backslash\{0\}$, in which case we say that $w$ is elliptic in $\mathcal{W}_{\xi}$.

It is an elementary result in homological algebra that, for the purpose of computing Euler-Poincaré pairings, one may replace any module by its semisimplification. Hence in the next theorem it suffices to compute $E P_{\mathcal{H}}\left(\pi, \pi^{\prime}\right)$ for $\pi, \pi^{\prime} \in \operatorname{Mod}_{f, \mathcal{W} \xi}(\mathcal{S})$ completely reducible. Recall that irreducible tempered modules are unitarizable [DeOp1, Corollary 3.23]. In particular Theorem 4.1, d applies to $\pi$ and $\pi^{\prime}$.

Theorem 6.5. Let $\pi, \pi^{\prime} \in \operatorname{Mod}_{f, u n, \mathcal{W} \xi}(\mathcal{S})$, as in Theorem 4.1. d. Denote by $\Phi_{\xi}^{*}(\pi)=$ $\operatorname{Hom}_{\mathcal{H}}(\pi, \pi(\xi))$ and $\Phi_{\xi}^{*}\left(\pi^{\prime}\right)=\operatorname{Hom}_{\mathcal{H}}\left(\pi^{\prime}, \pi(\xi)\right)$ the corresponding modules of $\mathbb{C}\left[\mathfrak{R}_{\xi}, \kappa_{\xi}\right]$. Then

$$
E P_{\mathcal{H}}\left(\pi, \pi^{\prime}\right)=\left|\mathfrak{R}_{\xi}\right|^{-1} \sum_{r \in \mathfrak{R}_{\xi}} \operatorname{det}(1-r)_{T_{\xi}\left(\Xi_{u n}\right)} \operatorname{tr}_{\Phi_{\xi}^{*}(\pi)}(r) \overline{\operatorname{tr}_{\Phi_{\xi}^{*}\left(\pi^{\prime}\right)}(r)}
$$


Proof. By (76)

$$
\begin{aligned}
E P_{\mathcal{H}}\left(\pi, \pi^{\prime}\right) & =\sum_{n \geq 0}(-1)^{n} \operatorname{dim}_{\mathbb{C}}\left(\Phi_{\xi}^{*}(\pi) \otimes_{\mathbb{C}} \Phi_{\xi}\left(\pi^{\prime}\right) \otimes_{\mathbb{R}} \bigwedge^{n} E_{\xi}^{*}\right)^{\mathfrak{R}_{\xi}^{*}} \\
& =\sum_{n \geq 0}(-1)^{n}\left|\mathfrak{R}_{\xi}^{*}\right|^{-1} \sum_{r \in \mathfrak{R}_{\xi}^{*}} \operatorname{tr}_{\Phi_{\xi}^{*}(\pi)}(r) \operatorname{tr}_{\Phi_{\xi}\left(\pi^{\prime}\right)}(r) \operatorname{tr} \bigwedge^{n} E_{\xi}^{*}(r) \\
& =\left|\Re_{\xi}^{*}\right|^{-1} \sum_{r \in \Re_{\xi}^{*}} \operatorname{tr}_{\Phi_{\xi}^{*}(\pi)}(r) \overline{\operatorname{tr}_{\Phi_{\xi}^{*}\left(\pi^{\prime}\right)}(r)} \sum_{n \geq 0}(-1)^{n} \operatorname{tr}^{n} \bigwedge^{n} E_{\xi}^{*}(r) \\
& =\left|\mathfrak{R}_{\xi}^{*}\right|^{-1} \sum_{r \in \Re_{\xi}^{*}} \operatorname{tr}_{\Phi_{\xi}^{*}(\pi)}(r) \overline{\operatorname{tr}_{\Phi_{\xi}^{*}\left(\pi^{\prime}\right)}(r)} \operatorname{det}(1-r)_{E_{\xi}^{*}} .
\end{aligned}
$$

Notice that this formula does not use the entire action of $\mathfrak{R}_{\xi}^{*}$ on $E_{\xi}^{*}$, only $\operatorname{det}(1-r)_{E_{\xi}^{*}}$. This determinant is zero whenever $r \in \mathfrak{R}_{\xi}^{*}$ fixes a nonzero vector in $E_{\xi}^{*}$. Suppose that $R_{\xi}$ is nonempty. Then $R_{\xi}^{\vee}$ is a nonempty root system in $\mathfrak{a}^{P}$ and $\mathbb{R} R_{\xi}^{\vee}$ can be identified with a subspace of $E_{\xi}^{*}$. Every $r \in \mathfrak{R}_{\xi}^{*}$ fixes $\sum_{\alpha \in R_{\xi}^{+}} \alpha^{\vee} \in \mathfrak{a}^{P} \backslash\{0\}$, so $r$ fixes nonzero vectors of $T_{t}\left(U_{\xi}\right)$ and $E_{\xi}^{*}$. We conclude that

$$
\operatorname{det}(1-r)_{E_{\xi}^{*}}=0=\operatorname{det}(1-r)_{T_{t}\left(U_{\xi}\right)}
$$

whenever $R_{\xi}$ is nonempty. Therefore we may always replace $E_{\xi}^{*}$ by $T_{t}\left(U_{\xi}\right)=T_{\xi}\left(\Xi_{u n}\right)$ in (80). So by (75) and (80)

$$
E P_{\mathcal{H}}\left(\pi, \pi^{\prime}\right)=\left|\mathfrak{R}_{\xi}^{*}\right|^{-1} \sum_{r \in \mathfrak{R}_{\xi}^{*}} \operatorname{det}(1-r)_{T_{\xi}\left(\Xi_{u n}\right)} \operatorname{tr}_{\Phi_{\xi}^{*}(\pi)}(r) \overline{\operatorname{tr}_{\Phi_{\xi}^{*}\left(\pi^{\prime}\right)}(r)} .
$$

Finally we want to reduce from $\mathfrak{R}_{\xi}^{*}$ to $\mathfrak{R}_{\xi}$. The action of $\mathfrak{R}_{\xi}^{*}$ on $T_{\xi}\left(\Xi_{\text {un }}\right)$ is defined via the quotient map $\mathfrak{R}_{\xi}^{*} \rightarrow \mathfrak{R}_{\xi}$, so that is no problem. By (172) the $\mathfrak{R}_{\xi}^{*}$-representation $\Phi_{\xi}^{*}(\pi) \otimes \Phi_{\xi}\left(\pi^{\prime}\right)$ is actually a representation of $\mathfrak{R}_{\xi}$, with trace

$$
\operatorname{tr}_{\Phi_{\xi}^{*}(\pi) \otimes \Phi_{\xi}^{*}\left(\pi^{\prime}\right)^{*}}(r)=\operatorname{tr}_{\Phi_{\xi}^{*}(\pi)}(r) \overline{\operatorname{tr}_{\Phi_{\xi}^{*}\left(\pi^{\prime}\right)}(r)} .
$$

Hence any two elements of $\mathfrak{R}_{\xi}^{*}$ with the same image in $\mathfrak{R}_{\xi}$ give the same contribution to (81).

The next result follows from Theorem 6.4 but it is also interesting to derive it from Theorem 6.5. since that proof can be generalized to reductive groups.

Corollary 6.6. Let $\xi=(P, \delta, t) \in \Xi_{\text {un }}$ and let $\chi \in G_{\mathbb{C}}\left(\operatorname{Mod}_{f, u n, \mathcal{W} \xi}(\mathcal{S})\right) \subset G_{\mathbb{C}}(\mathcal{H})$. Then $\operatorname{EP}_{\mathcal{H}}(\chi, \chi)=0$ if and only if $\chi \in \sum_{P \subset Q \subset F_{0}, \mathbb{R} Q \neq \mathfrak{a}^{*}} \operatorname{Ind}_{\mathcal{H}^{Q}}^{\mathcal{H}}\left(G_{\mathbb{C}}\left(\mathcal{H}^{Q}\right)\right)$.

Proof. The formula (81) says (by definition) that $E P_{\mathcal{H}}\left(\chi, \chi^{\prime}\right)$ is the elliptic pairing $e_{\mathfrak{R}_{\xi}^{*}}\left(\Phi_{\xi}\left(\chi^{\prime}\right), \Phi_{\xi}(\chi)\right)$ of trace functions on $\mathfrak{R}_{\xi}^{*}$ with respect to the $\mathfrak{R}_{\xi}^{*}$-representation $T_{\xi}\left(\Xi_{u n}\right)$, in the sense of Reeder [Ree]. By [Ree, Proposition 2.2.2] the radical of the Hermitian form $e_{\mathfrak{R}_{\xi}^{*}}$ is $\sum_{\Gamma} \operatorname{Ind}_{\Gamma}^{\mathfrak{R}_{\xi}^{*}}\left(G_{\mathbb{C}}(\Gamma)\right)$, where the sum runs over all subgroups $\Gamma \subset R_{\xi}^{*}$ for which $T_{\xi}\left(\Xi_{u n}\right)^{\Gamma} \neq 0$. Clearly it suffices to consider $\Gamma$ 's that contain the central subgroup $Z_{\xi}=\operatorname{ker}\left(\mathfrak{R}_{\xi}^{*} \rightarrow \mathfrak{R}_{\xi}\right)$. From (81) we see that $\mathfrak{R}_{\xi}^{*}$-representations with different $Z_{\xi^{-}}$character are orthogonal for $e_{\mathfrak{R}_{\xi}^{*}}$, so the above remains valid if we restrict to $p G_{\mathbb{C}}\left(\Re_{\xi}^{*}\right)=G_{\mathbb{C}}\left(\mathbb{C}\left[\Re_{\xi}, \kappa_{\xi}\right]\right)$. In particular

$$
e_{\mathfrak{R}_{\xi}^{*}}\left(\Phi_{\xi}(\chi), \Phi_{\xi}(\chi)\right)=0 \Longleftrightarrow \Phi_{\xi}(\chi) \in \sum_{\Gamma^{*}} \operatorname{Ind}_{p \mathbb{C}\left[\Gamma^{*}\right]}^{p \mathbb{C}\left[\mathfrak{R}^{*}\right]}\left(G_{\mathbb{C}}\left(p \mathbb{C}\left[\Gamma^{*}\right]\right)\right),
$$


where $Z_{\xi} \subset \Gamma^{*} \subset \mathfrak{R}_{\xi}^{*}$ and $T_{\xi}\left(\Xi_{u n}\right)^{\Gamma^{*}} \neq 0$. Since $\mathfrak{R}_{\xi}$ is built from elements of the

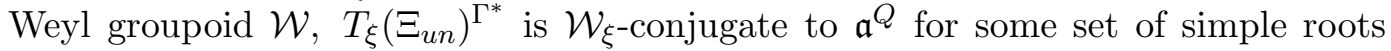
$Q \supset P$. In view of (49) and (53) this means that $\Gamma^{*} / Z_{\xi}$ is conjugate to a subgroup of $\mathfrak{R}_{\xi}^{Q}$. Thus the right hand side of (82) becomes

$$
\Phi_{\xi}(\chi) \in \sum_{P \subset Q \subset F_{0}, \mathfrak{a} q \neq 0} \operatorname{Ind}_{\mathbb{C}\left[\Re_{\xi}^{Q}, \kappa_{\xi}^{Q}\right]}^{\mathbb{C}\left[\Re_{\xi}, \kappa_{\xi}\right]}\left(G_{\mathbb{C}}\left(\mathbb{C}\left[\Re_{\xi}^{Q}, \kappa_{\xi}^{Q}\right]\right)\right) .
$$

Theorem 4.1,d implies that

$$
\Phi_{\xi}^{Q}: G_{\mathbb{C}}\left(\operatorname{Mod}_{f, u n, \mathcal{W}}{ }^{Q} \xi\left(\mathcal{S}\left(\mathcal{R}^{Q}, q\right)\right)\right) \rightarrow G_{\mathbb{C}}\left(\mathbb{C}\left[\Re_{\xi}^{Q}, \kappa_{\xi}^{Q}\right]\right)
$$

is bijective, which together with (154) allows us to rephrase (82) in $G_{\mathbb{C}}(\mathcal{H})$ as

$$
E P_{\mathcal{H}}(\chi, \chi)=0 \Longleftrightarrow \chi \in \sum_{P \subset Q \subset F_{0}, \mathfrak{a}^{Q} \neq 0} \operatorname{Ind}_{\mathcal{H}^{Q}}^{\mathcal{H}}\left(G_{\mathbb{C}}\left(\operatorname{Mod}_{f, u n, \mathcal{W}^{Q} \xi}\left(\mathcal{S}\left(\mathcal{R}^{Q}, q\right)\right)\right)\right) .
$$

Finally we note that the condition $\mathfrak{a}^{Q} \neq 0$ is equivalent to $\mathbb{R} Q \neq \mathfrak{a}^{*}$.

\section{The CASE Of ReduCtive $p$-ADiC Groups}

Here we discuss how the proofs of our main results can be adjusted so that they apply to tempered representations of reductive groups over local non-archimedean fields.

Let $L$ be a reductive $p$-adic group, let $\mathcal{H}(L)$ be its Hecke algebra and let $\operatorname{Mod}(\mathcal{H}(L))$ be the category of smooth $L$-representations. Let $K \subset L$ be any compact open subgroup and consider the subalgebra $\mathcal{H}(L, K)$ of $K$-biinvariant functions in $\mathcal{H}(L)$. According to BeDe, Section 3] there exist arbitrarily small "good" compact open $K$ such that $\operatorname{Mod}(\mathcal{H}(L, K))$ is equivalent to the category consisting of those smooth $L$-representations that are generated by their $K$-invariant vectors. Here the adjective good means that the latter category is a Serre subcategory of $\operatorname{Mod}(\mathcal{H}(L))$. It also is known from $\mathrm{BeDe}$ that these subcategories exhaust $\operatorname{Mod}(\mathcal{H}(L))$.

The tempered smooth $L$-representations are precisely those smooth representations that extend in a continuous way to modules over the Harish-Chandra-Schwartz algebra $\mathcal{S}(L)$. All extensions of admissible $\mathcal{S}(L)$-modules can be studied with subalgebras $\mathcal{S}(L, K)$ of $K$-biinvariant functions, see [ScZi1, OpSo3. Clearly (23) is similar to the Plancherel isomorphism for the $\mathcal{S}(L)$ [ScZi2, Wal]. One can easily deduce from Wal that the subalgebra $\mathcal{S}(L, K)$ has exactly the same shape as (24), see [Sol1, Theorem 10].

Suppose that $V \in \operatorname{Mod}(\mathcal{S}(L))$ is admissible. By comparing explicit projective resolutions of $V$ as an $\mathcal{H}(L)$-module and as an $\mathcal{S}(L)$-module, it is shown in OpSo3, Proposition 4.3.a] that

$$
\operatorname{Ext}_{\mathcal{H}(L)}^{n}\left(V, V^{\prime}\right) \cong \operatorname{Ext}_{\mathcal{S}(L)}^{n}\left(V, V^{\prime}\right) \quad \text { for all } \quad V^{\prime} \in \operatorname{Mod}(\mathcal{S}(L))
$$

Here we work in the category of all modules over $\mathcal{H}(L)$ or $\mathcal{S}(L)$, as advocated by Schneider and Zink [ScZi1, ScZi2. Assume that $V$ and $V^{\prime}$ are generated by their $K$-invariant vectors for some good compact open subgroup $K$. Then OpSo3, Proposition 4.3.b] says that (83) is also isomorphic to $\operatorname{Ext}_{S(L, K)}^{n}\left(V^{K}, V^{\prime K}\right)$. In case 
that moreover $V^{\prime K}$ is a Fréchet $\mathcal{S}(L, K)$-module, OpSo3, Proposition 4.3.c] provides a natural isomorphism

$$
\operatorname{Ext}_{S(L, K)}^{n}\left(V^{K}, V^{\prime K}\right) \cong \operatorname{Ext}_{\operatorname{Mod}_{F r}(S(L, K))}^{n}\left(V^{K}, V^{\prime K}\right) .
$$

Here the subscript 'Fr' indicates the category of Fréchet modules with exact sequences that are linearly split. These results can play the role of OpSo1, Corollary $3.7]$ in the proofs of Corollary 2.6 and Theorem 5.2 for $p$-adic groups.

Alternatively, one can consider $\mathcal{S}(L)$ as a bornological algebra. It is natural to endow $\mathcal{S}(L)$ with the precompact bornology and $\mathcal{H}(G)$ with the fine bornology, see Mey2. For all bornological $\mathcal{S}(L)$-modules $V, V^{\prime}$ and all $n \in \mathbb{Z}_{\geq 0}$

$$
\operatorname{Ext}_{\operatorname{Mod}_{b o r}(\mathcal{H}(L))}^{n}\left(V, V^{\prime}\right) \cong \operatorname{Ext}_{\operatorname{Mod}_{b o r}(\mathcal{S}(L))}^{n}\left(V, V^{\prime}\right)
$$

by [Mey2, Theorem 21]. It follows quickly from the definition of the fine bornology Mey2, pp. 364-365] that

$$
\operatorname{Ext}_{\mathcal{H}(L)}^{n}\left(V, V^{\prime}\right) \cong \operatorname{Ext}_{\operatorname{Mod}_{b o r}(\mathcal{H}(L))}^{n}\left(V, V^{\prime}\right) \quad \text { if } V \text { is admissible. }
$$

Let $P$ be a parabolic subgroup and $M$ a Levi factor of $P$. Let $\sigma$ be an irreducible smooth unitary $M$-representation which is square-integrable modulo the center of $M$. The $L$-representation $\mathcal{I}_{P}^{L}(\sigma)$, the smooth normalized parabolic induction of $\sigma$, plays the role of $\pi(\xi)$. Choose good compact open subgroups $K_{i} \subset L$ such that $\mathcal{I}_{P}^{L}(\sigma)$ is generated by its $K_{i}$-invariant vectors. We may assume that the $K_{i}$ decrease when $i \in \mathbb{N}$ increases and that $\bigcap_{i} K_{i}=\{1\}$, so $\mathcal{S}(L)=\bigcup_{i} \mathcal{S}\left(L, K_{i}\right)$. Harish-Chandra's Plancherel isomorphism for $L$ shows that $\mathcal{S}(L)$ contains a direct summand

$$
\mathcal{S}(L)^{\sigma}=\bigcup_{i} \mathcal{S}\left(L, K_{i}\right)^{\sigma}
$$

which governs all tempered $L$-representations in the block determined by $\sigma$. Moreover the algebras $\mathcal{S}\left(L, K_{i}\right)^{\sigma}$ are nuclear Fréchet of the form (33), and they are all Morita equivalent. The algebra $\mathcal{S}(L)^{\sigma}$ is Morita equivalent to $\mathcal{S}\left(L, K_{i}\right)^{\sigma}$ via the bimodules $\mathcal{S}(L)^{\sigma} e_{K_{i}}$ and $e_{K_{i}} \mathcal{S}(L)^{\sigma}$, where $e_{K_{i}} \in \mathcal{H}(L)$ is the idempotent associated to $K_{i}$. However, $\mathcal{S}(L)^{\sigma}$ is not a Fréchet algebra, only an inductive limit of Fréchet algebras.

All the algebras in (86) have the same center, which by Wal is isomorphic to $C^{\infty}(T)^{\mathcal{W}}$ for a suitable compact torus $T$ and a finite group $\mathcal{W}$. The representation $\sigma$ determines a point of $T$ and an orbit $\mathcal{W} \sigma \subset T$. Let $m_{\mathcal{W} \sigma}^{\infty} \subset C^{\infty}(T)^{\mathcal{W}}$ be the ideal of functions that are flat at $\mathcal{W} \sigma$. We define

$$
\begin{aligned}
& \widehat{\mathcal{S}(L)}_{\mathcal{W} \sigma}=\mathcal{S}(L)^{\sigma} /{\overline{m_{\mathcal{W} \sigma}^{\infty} \mathcal{S}(L)^{\sigma}}}^{\mathcal{L}}, \\
& \operatorname{Mod}_{\text {bor }}^{\mathcal{W} \sigma \text { tor }}(\mathcal{S}(L))=\left\{V \in \operatorname{Mod}_{\text {bor }}(\mathcal{S}(L)): m_{\mathcal{W} \xi}^{\infty} V=0\right\} .
\end{aligned}
$$

All the results from Sections 2 and 3 also hold for these objects, with some obvious changes of notation. We have to be careful only when we want to apply Theorem 3.2 to $\mathcal{S}(L)^{\sigma}$. The complication is that over there we work with a finite dimensional representation $V$ of some finite group $G$, which in the case under consideration is a central extension $\mathfrak{R}_{\sigma}^{*}$ of the R-group $\mathfrak{R}_{\sigma}$ from [Sil]. That is enough for $\mathcal{S}\left(L, K_{i}\right)^{\sigma}$, but for $\mathcal{S}(L)^{\sigma}$ we are naturally lead to the infinite dimensional module $V=\mathcal{I}_{P}^{L}(\sigma)$. Then we must replace the bimodules (34) by

$$
\begin{aligned}
& D_{1}=\lim _{i \rightarrow \infty} C^{\infty}(T) \otimes \operatorname{Hom}_{\mathbb{C}}\left(\mathcal{I}_{P}^{L}(\sigma)_{K_{i}}, V^{\prime}\right), \\
& D_{2}=\lim _{i \rightarrow \infty} C^{\infty}(T) \otimes \operatorname{Hom}_{\mathbb{C}}\left(V^{\prime}, \mathcal{I}_{P}^{L}(\sigma)^{K_{i}}\right),
\end{aligned}
$$


where the subscript $K_{i}$ means coinvariants and the superscript $K_{i}$ means invariants. In view of (86), the proof of Theorem 3.2 goes through.

The proof of Theorem 5.2 relies on two deep results: the Knapp-Stein linear independence theorem (Theorem 4.1) and the Plancherel isomorphism for $\mathcal{S}(23)$. These also hold for the algebras $\mathcal{S}(L), \mathcal{S}(L)^{\sigma}$ and $\mathcal{S}\left(L, K_{i}\right)^{\sigma}$, as we indicated above, so our proof remains valid. We obtain equivalences of exact categories

$$
\operatorname{Mod}_{\text {bor }}^{\mathcal{W} \sigma, t o r}(\mathcal{S}(L)) \cong \operatorname{Mod}_{\text {bor }}\left(\widehat{\mathcal{S}(L)}_{\mathcal{W} \sigma}\right) \cong \operatorname{Mod}_{\text {bor }}\left(p_{\sigma}\left(\widehat{S\left(E_{\sigma}\right)} \rtimes \mathfrak{R}_{\sigma}^{*}\right)\right)
$$

We note however that these equivalences do not preserve Fréchet modules, essentially because $\mathcal{S}(L)$ is too large to admit enough such modules. The first part of (88) does not change the modules, the second part comes from a Morita equivalence of bornological algebras. To find the Morita bimodules, we start with (87). From the proof of Theorem 3.2 we see that we must take $\mathfrak{R}_{\sigma}^{*}$-invariants and that we have to replace $C^{\infty}(T)$ by a formal power series ring, which is none other than $\widehat{S\left(E_{\sigma}\right)}$. So the functor from left to right in (87) is given by the bornological tensor product with

$$
D=\lim _{i \rightarrow \infty}\left(\widehat{S\left(E_{\sigma}\right)} \otimes \operatorname{Hom}_{\mathbb{C}}\left(\mathcal{I}_{P}^{L}(\sigma)_{K_{i}}, p_{\sigma} \mathbb{C}\left[\mathfrak{R}_{\sigma}^{*}\right]\right)\right)^{\mathfrak{R}_{\sigma}^{*}}
$$

over $\widehat{\mathcal{S}(L)}_{\mathcal{W} \sigma}$. For the opposite direction we can tensor with the bimodule

$$
D^{\vee}=\lim _{i \rightarrow \infty}\left(\widehat{S\left(E_{\sigma}\right)} \otimes \operatorname{Hom}_{\mathbb{C}}\left(p_{\sigma} \mathbb{C}\left[\mathfrak{R}_{\sigma}^{*}\right], \mathcal{I}_{P}^{L}(\sigma)^{K_{i}}\right)\right)^{\mathfrak{R}_{\sigma}^{*}}
$$

over the algebra

$p_{\sigma}\left(\widehat{S\left(E_{\sigma}\right)} \rtimes \mathfrak{R}_{\sigma}^{*}\right) \cong\left(\widehat{S\left(E_{\sigma}\right)} \otimes \operatorname{End}_{\mathbb{C}}\left(p_{\sigma} \mathbb{C}\left[\mathfrak{R}_{\sigma}^{*}\right]\right)\right)^{\mathfrak{R}_{\sigma}^{*}}=\left(\widehat{S\left(E_{\sigma}\right)} \otimes \operatorname{End}_{\mathbb{C}}\left(\mathbb{C}\left[\Re_{\sigma}, \kappa_{\sigma}^{-1}\right]\right)\right)^{\mathfrak{R}_{\sigma}^{*}}$.

To compute the Ext-groups we need the fundamental result (85). Using that the proof of Theorem 5.2 goes through, thus establishing Theorem 3 .

Now it is clear that our proof of 6.5 is also valid for $L$. To formulate the result, consider the real Lie algebra $\operatorname{Hom}\left(X^{*}(M), \mathbb{R}\right)$ of the center of $M$. The group $\mathfrak{R}_{\sigma}$ acts on $\operatorname{Hom}\left(X^{*}(M), \mathbb{R}\right)$ and we denote by $d(r)$ the determinant of the linear transformation $1-r$. Let $\pi$ be a finite length unitary tempered $L$-representation all whose irreducible constituents occur in $\mathcal{I}_{P}^{L}(\sigma)$ and let $\rho=\operatorname{Hom}_{L}\left(\pi, \mathcal{I}_{P}^{L}(\sigma)\right)$ be the projective $\mathfrak{R}_{\sigma}$-representation associated to it via the Knapp-Stein Theorem. Then

$$
E P_{L}\left(\pi, \pi^{\prime}\right)=\left|\mathfrak{R}_{\sigma}\right|^{-1} \sum_{r \in \mathfrak{R}_{\sigma}}|d(r)| \operatorname{tr}_{\rho}(r) \overline{\operatorname{tr}_{\rho^{\prime}}(r)} .
$$

To relate this to Kazhdan's elliptic pairing we need some additional properties of the Euler-Poincaré pairing in $\operatorname{Mod}(\mathcal{H}(L))$. Recall that $G_{\mathbb{C}}(L)$ is the Grothendieck group of the category of admissible $L$-representations, tensored with $\mathbb{C}$. Since the elliptic pairing $e_{L}\left(\pi, \pi^{\prime}\right)$ depends only on the traces of $\pi$ and $\pi^{\prime}$, it factors via the canonical map from admissible representations to the Grothendieck group. By standard homological algebra $E P_{L}$ has the same property. We extend pairings to $G_{\mathbb{C}}(L)$ by making them conjugate linear the first argument and linear in the second.

Lemma 7.1. Suppose that the center $Z(L)$ of $L$ is compact. Then $G_{\mathbb{C}}(L)$ is spanned by the union of all irreducible tempered $L$-representations and all representations parabolically induced from proper Levi subgroups of $L$.

Proof. This follows from the Langlands classification, for which we refer to BoWa, Section XI.2] and [Kon]. The argument is analogous to the proof of Lemma 6.3. 
The next result is known from [ScSt, Lemma III.4.18], but the authors found it useful to have a simpler proof that does not depend on the projective resolutions constructed in $\mathrm{ScSt}$.

Proposition 7.2. (a) Let $P$ be a parabolic subgroup of $L$ with Levi factor $M$, such that $Z(M)$ is not compact. Then $\mathcal{I}_{P}^{L}\left(G_{\mathbb{C}}(M)\right)$ lies in the radical of $E P_{L}$.

(b) $E P_{L}$ is a Hermitian form on $G_{\mathbb{C}}(L)$.

(c) $E P_{L}$ is positive semidefinite and its radical is $\sum_{P, M} \mathcal{I}_{P}^{L}\left(G_{\mathbb{C}}(M)\right)$, where the sum runs over $P$ and $M$ as in part (a).

Proof. (a) Since we do not yet know that $E P_{L}$ is Hermitian, we have to deal with both its left and its right radical. According to [Bez, Claim 4.3], an elementary argument which Bezrukavnikov ascribes to Bernstein,

$$
E P_{L}=0 \text { if } Z(L) \text { is not compact. }
$$

Let $V, W$ be smooth $L$-representations and $V^{\prime}, W^{\prime}$ admissible smooth $M$-representations. By Frobenius reciprocity

$$
\operatorname{Ext}_{L}^{n}\left(V, \mathcal{I}_{P}^{L}\left(W^{\prime}\right)\right) \cong \operatorname{Ext}_{M}^{n}\left(r_{P}^{L}(V), W^{\prime}\right),
$$

where $r_{P}^{L}$ denotes Jacquet's restriction functor. As $M$ is a proper Levi subgroup of $L$, its center is not compact, so (92) and (93) show that $\mathcal{I}_{P}^{L}\left(W^{\prime}\right)$ is in the right radical of $E P_{L}$. Now we could use Bernstein's second adjointness theorem to reach to same conclusion for the left radical, but we prefer to do without that deep result.

Let $\tilde{V}$ denote the contragredient representation of $V$, that is, the smooth part of the algebraic dual space of $V$. The functor $V \mapsto \tilde{V}$ is exact and for admissible representations $\tilde{\tilde{V}} \cong V$. Suppose that $V, W$ are admissible and that

$$
0 \rightarrow W \rightarrow V_{n} \rightarrow \cdots \rightarrow V_{1} \rightarrow V \rightarrow 0
$$

is an $n$-fold extension in $\operatorname{Mod}(\mathcal{H}(L))$. The contragredience functor yields $n$-fold extensions

$$
\begin{aligned}
& 0 \rightarrow \tilde{V} \rightarrow \tilde{V}_{1} \rightarrow \cdots \rightarrow \tilde{V}_{n} \rightarrow \tilde{W} \rightarrow 0, \\
& 0 \rightarrow W \rightarrow \tilde{\tilde{V}}_{n} \rightarrow \cdots \rightarrow \tilde{\tilde{V}}_{1} \rightarrow V \rightarrow 0 .
\end{aligned}
$$

The existence of a natural inclusion $V_{j} \rightarrow \tilde{\tilde{V}}_{j}$ means that (96) is equivalent to (94) in the sense of Yoneda extensions. Hence the functors $V_{j} \mapsto \tilde{\tilde{V}}_{j}$ and $V_{j} \mapsto \tilde{V}_{j}$ induce isomorphisms between the corresponding Yoneda Ext-groups. Since $\operatorname{Mod}(\mathcal{H}(L))$ has enough projectives, we may also phrase this with the derived functors of $\operatorname{Hom}_{L}$ :

$$
\operatorname{Ext}_{L}^{n}(V, W) \cong \operatorname{Ext}_{L}^{n}(\tilde{W}, \tilde{V})
$$

By (97), Cas, Proposition 3.1.2] and (93)

$$
\operatorname{Ext}_{L}^{n}\left(\mathcal{I}_{P}^{L}\left(V^{\prime}\right), W\right) \cong \operatorname{Ext}_{L}^{n}\left(\tilde{W}, \widetilde{\mathcal{I}_{P}^{L}\left(V^{\prime}\right)}\right) \cong \operatorname{Ext}_{L}^{n}\left(\tilde{W}, \mathcal{I}_{P}^{L}\left(\tilde{V}^{\prime}\right)\right) \cong \operatorname{Ext}_{M}^{n}\left(r_{P}^{L}(\tilde{W}), \tilde{V}^{\prime}\right) .
$$

Now (92) shows that $\mathcal{I}_{P}^{L}\left(V^{\prime}\right)$ is in the left radical of $E P_{L}$.

(b) By part (a) and Lemma [7.1 it suffices to check that $E P_{L}$ is symmetric for tempered admissible $L$-representations. Since this pairing factors via the Grothendieck group we may moreover replace every representation by its semisimplification. Such tempered representations $V, W$ are unitary by [Wal, Prop III.4.1], which implies that 
$V$ (resp. $W$ ) is isomorphic to the contragredient of the conjugate representation $\bar{V}$ (resp. $\bar{W}$ ). Using (97) we conclude that

$$
E P_{L}(V, W)=E P_{L}(\bar{V}, \bar{W})=E P_{L}(\tilde{\bar{W}}, \tilde{\bar{V}})=E P_{L}(W, V)
$$

(c) By (85) tempered representations with different $Z(\mathcal{S}(L))$-characters are orthogonal for $E P_{L}$. Thus we only have to check positive definiteness for the Grothendieck group of finite length unitary $\mathcal{S}(L)$-representations with one fixed $Z(\mathcal{S}(L))$-character, modulo the span of representations that are parabolically induced from the indicated Levi subgroups. In this setting the proof of Corollary [6.6 applies, all the required properties of R-groups are provided by [Art, Section 2]. That determines the radical, while (91) shows that $E P_{L}$ is positive semidefinite.

Recall the elliptic pairing $e_{L}$ on $G_{\mathbb{C}}(L)$ from [Kaz and (3).

Theorem 7.3. Suppose that the local non-archimedean field underlying $L$ has characteristic 0. Then $\operatorname{EP}_{L}\left(\pi, \pi^{\prime}\right)=e_{L}\left(\pi, \pi^{\prime}\right)$ for all admissible L-representations.

Proof. According to [Kaz, Theorem A] and Proposition [7.2.c the Hermitian forms $e_{L}$ and $E P_{L}$ have the same radical. Reasoning as in the proof of Proposition 7.2.b, it suffices to check the equality $E P_{L}\left(\pi, \pi^{\prime}\right)=e_{L}\left(\pi, \pi^{\prime}\right)$ for irreducible tempered $L$-representations $\pi, \pi^{\prime}$. This follows from (91) and [Art, Corollary 6.3].

\section{REFERENCES}

[Art] J. Arthur, "On elliptic tempered characters", Acta. Math. 171 (1993), 73-130

[BeDe] J.N. Bernstein, P. Deligne, "Le "centre" de Bernstein", pp. 1-32 in: Représentations des groupes réductifs sur un corps local, Travaux en cours, Hermann, 1984

[Bez] R. Bezrukavnikov, "Homological properties of representations of $p$-adic groups related to geometry of the group at infinity", PhD. Thesis, Tel-Aviv University, 1998, arXiv:math.RT/0406223

[BoWa] A. Borel, N.R. Wallach, Continuous cohomology, discrete subgroups, and representations of reductive groups, Annals of Mathematics Studies 94, Princeton University Press, Princeton NJ, 1980

[BuKu] C.J. Bushnell, P.C. Kutzko, "Types in reductive $p$-adic groups: the Hecke algebra of a cover", Proc. Amer. Math. Soc. 129.2 (2001), 601-607.

[Cas] W. Casselman, "Introduction to the theory of admissible representations of $p$-adic reductive groups", preprint, 1995

[Che] C.C. Chevalley, "Invariants of finite groups generated by reflections", American J. Math. 77.4 (1955), 778-782

[CuRe] C.W. Curtis, I. Reiner, Representation theory of finite groups and associative algebras, Pure and Applied Mathematics 11, John Wiley \& Sons, 1962

[DeOp1] P. Delorme, E.M. Opdam, "The Schwartz algebra of an affine Hecke algebra", J. reine angew. Math. 625 (2008), 59-114

[DeOp2] P. Delorme, E.M. Opdam, "Analytic R-groups of affine Hecke algebras", arXiv:0909.1227, 2009, to appear in J. reine angew. Math.

[Eis] D. Eisenbud, Commutative algebra with a view to algebraic geometry, Graduate Texts in Mathematics 150, Springer Verlag, 1995

[HC] Harish-Chandra, "Harmonic analysis on real reductive groups III. The Maass-Selberg relations and the Plancherel formula", Ann. of Math. 104 (1976), 117-201.

[Hei] V. Heiermann, "Opérateurs d'entrelacement et algébres de Hecke avec paramètres d'un groupe réductif $p$-adique - le cas des groupes classiques", Selecta Math. 17 (2011), doi: 10.1007/s00029011-0056-0

[Hum] J.E. Humphreys, Reflection groups and Coxeter groups, Cambridge Studies in Advanced Mathematics 29, Cambridge University Press, 1990 
[Kaz] D. Kazhdan, "Cuspidal geometry of p-adic groups", J. Analyse Math. 47 (1986), 1-36

[Kel] B. Keller, "Chain complexes and stable categories", Manuscripta Math. 67.4 (1990), 379-417

[KnSt] A.W. Knapp, E.M. Stein, "Intertwining operators for semisimple groups II", Invent. Math. 60.1 (1980), 9-84

[Kon] T. Konno, "A note on the Langlands classification and irreducibility of induced representations of $p$-adic groups", Kyushu J. Math. 57 (2003), 383-409

[Kopp] M.K. Kopp, "Fréchet algebras of finite type", Arch. Math. 83.3 (2004), 217-228

[LePl] C.W. Leung, R.J. Plymen, "Arithmetic aspects of operator algebras", Compos. Math. 77.3 (1991), 293-311

[Lus1] G. Lusztig, "Affine Hecke algebras and their graded version", J. Amer. Math. Soc 2.3 (1989), 599-635

[Lus2] G. Lusztig, "Classification of unipotent representations of simple $p$-adic groups", Internat. Math. Res. Notices 11 (1995), 517-589.

[Mac] S. Mac Lane, Homology, Grundlehren der mathematischen Wissenschaften 114, SpringerVerlag, 1975

[MeVo] R. Meise, D. Vogt, Einführung in die Funktionalanalysis, Vieweg Studium: Aufbaukurs Mathematik 62, Friedr. Vieweg \& Sohn, 1992

[Mey1] R. Meyer, "Embeddings of derived categories of bornological modules", arXiv:math.FA/0410596, 2004

[Mey2] R. Meyer, "Homological algebra for Schwartz algebras of reductive $p$-adic groups", pp. 263300 in: Noncommutative geometry and number theory, Aspects of Mathematics E37, Vieweg Verlag, 2006

[Mor] L. Morris, "Level zero G-types", Compositio Math. 118 (1999), 135-157

[Oort] F. Oort, "Yoneda extensions in abelian categories", Math. Ann. 153 (1964), 227-235

[Opd1] E.M. Opdam, "On the spectral decomposition of affine Hecke algebras", J. Inst. Math. Jussieu 3.4 (2004), 531-648

[Opd2] E.M. Opdam, "Hecke algebras and harmonic analysis", pp. 1227-1259 in: Proceedings ICM Madrid 2006 Vol. II, European Mathematical Society Publishing House, 2006

[OpSo1] E.M. Opdam, M. Solleveld, "Homological algebra for affine Hecke algebras", Adv. Math. 220 (2009), 1549-1601

[OpSo2] E.M. Opdam, M. Solleveld, "Discrete series characters for affine Hecke algebras and their formal dimensions", Acta Math. 105 (2010), 105-187

[OpSo3] E.M. Opdam, M. Solleveld, "Extension of tempered representations of reductive $p$-adic groups", in preparation

[Poe] V. Poénaru, Singularités $C^{\infty}$ en Présence de Symétrie, Lecture Notes in Mathematics 510, Springer-Verlag, 1976.

[Qui] D. Quillen, "Higher algebraic K-theory", pp. 85-147 in: Algebraic KK-theory, I: Higher KKtheories, Lecture Notes in Mathematics 341, Springer-Verlag, 1973

[Ree] M. Reeder, "Euler-Poincaré pairings and elliptic representations of Weyl groups and $p$-adic groups", Compos. Math. 129 (2001), 149-181

[Roc] A. Roche, "Parabolic induction and the Bernstein decomposition", Compos. Math. 134.2 (2002), 113-133.

[ScSt] P. Schneider, U. Stuhler, "Representation theory and sheaves on the Bruhat-Tits building", Publ. Math. Inst. Hautes Études Sci. 85 (1997), 97-191

[ScZi1] P. Schneider, E.-W. Zink, "The algebraic theory of tempered representations of $p$-adic groups. Part I: Parabolic induction and restriction", J. Inst. Math. Jussieu 6 (2007), 639-688

[ScZi2] P. Schneider, E.-W. Zink, "The algebraic theory of tempered representations of $p$-adic groups. Part II: Projective generators", Geom. Func. Anal. 17.6 (2008), 2018-2065

[Sil] A.J. Silberger, "The Knapp-Stein dimension theorem for $p$-adic groups", Proc. Amer. Math. Soc. 68.2 (1978), 243-246, and "The Knapp-Stein dimension theorem for $p$-adic groups. Correction" Proc. Amer. Math. Soc. 76.1 (1979), 169-170

[Slo] K. Slooten, "Induced discrete series representations for Hecke algebras of types $B_{n}^{\text {aff }}$ and $C_{n}^{\text {aff " , }}$ Internat. Math. Res. Notices 10 (2008), doi: 10.1093/imrn/rnn023

[Sol1] M. Solleveld, "Some Fréchet algebras for which the Chern character is an isomorphism", K-Theory 36 (2005), 275-290 
[Sol2] M. Solleveld, Periodic cyclic homology of affine Hecke algebras, PhD Thesis, Universiteit van Amsterdam, 2007, arXiv:0910.1606

[Sol3] M. Solleveld "On the classification of irreducible representations of affine Hecke algebras with unequal parameters", arXiv:1008.0177, 2010, to appear in Representation Theory

[Tou] J-C. Tougeron, Idéaux de Fonctions Différentiables, Springer Verlag, 1972

[ToMe] J-C. Tougeron, J. Merrien, "Idéaux de Fonctions Différentiables II", Annales de l'institut Fourier, 20.1 (1970), 179-233

[Vogt] D. Vogt, "On the functors $\operatorname{Ext}^{1}(E, F)$ for Fréchet spaces", Studia Math. 85 (1987), 163-197

[VoWa] D. Vogt, M.J. Wagner, "Charakterisierung der Quotientenräume von $s$ und eine Vermutung von Martineau", Studia Math. 67 (1980), 225-240

[Wal] J.-L. Waldspurger, "La formule de Plancherel pour les groupes $p$-adiques (d'après HarishChandra)", J. Inst. Math. Jussieu 2.2 (2003), 235-333

[Was] A. Wassermann, "Cyclic cohomology of algebras of smooth functions on orbifolds", pp. 229244 in: Operator algebras and applications Vol. I, London Mathematical Society Lecture Notes 135, Cambridge University Press, 1988.

Korteweg-De Vries Institute for Mathematics, Universiteit van Amsterdam, Science Park 904, 1098 XH Amsterdam, The Netherlands

E-mail address: e.m.opdam@uva.nl

Institute for Mathematics, Astrophysiscs and Particle Physiscs, Radboud UniverSiteit Nijmegen, Heyendaalseweg 135, 6525AJ Nijmegen, The Netherlands

E-mail address: m.solleveld@science.ru.nl 